Master of Science Thesis

\title{
Flow visualization and force measurements on a hovering flapping-wing MAV \\ 'DelFly II'
}

Kristien M.E. De Clercq

April 2, 2009
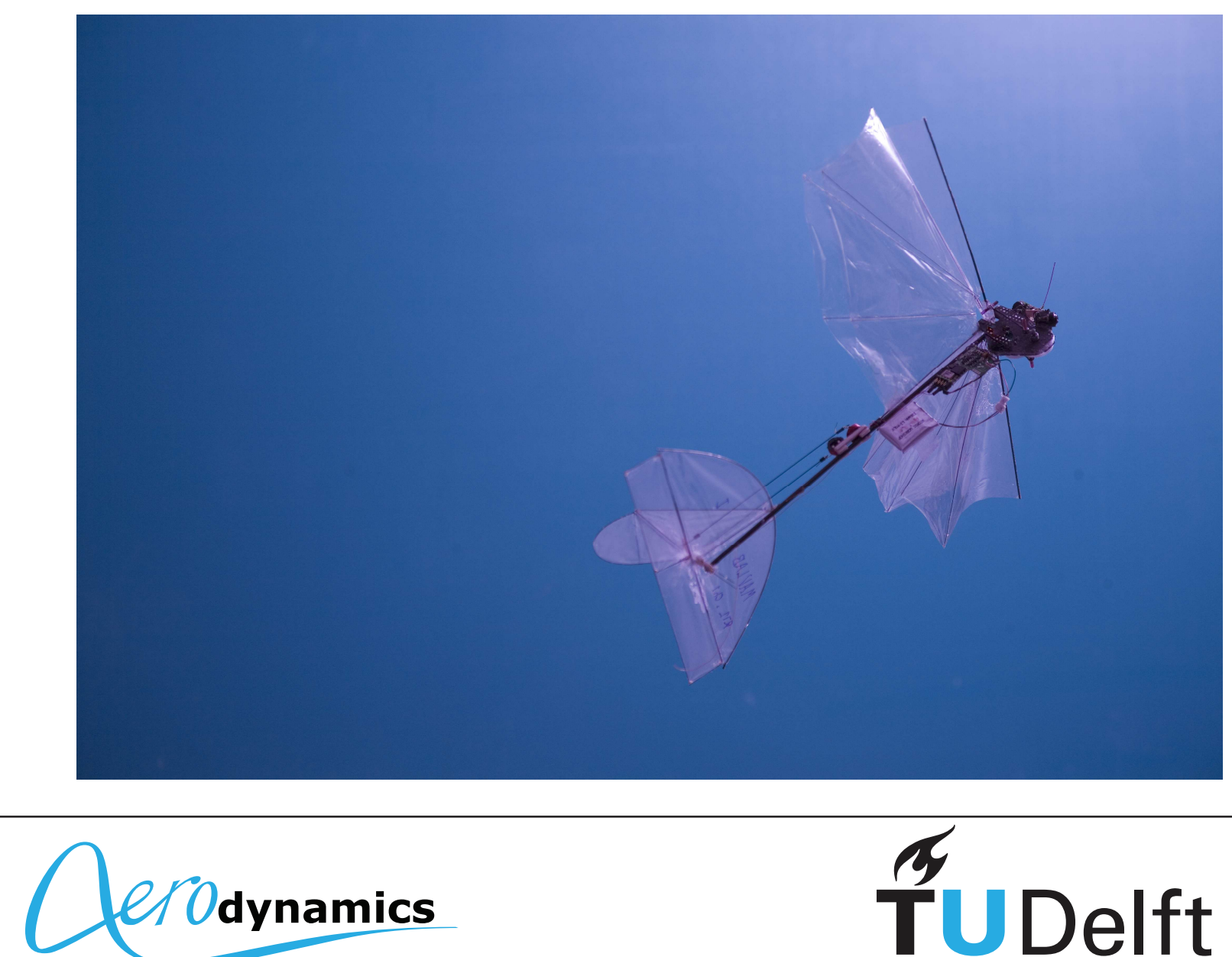



\title{
Flow visualization and force measurements on a hovering flapping-wing MAV 'DelFly II'
}

\author{
Master of Science Thesis \\ For obtaining the degree of Master of Science in Aerospace Engineering \\ at Delft University of Technology
}

Kristien M.E. De Clercq

April 2, 2009

Faculty of Aerospace Engineering - Delft University of Technology 


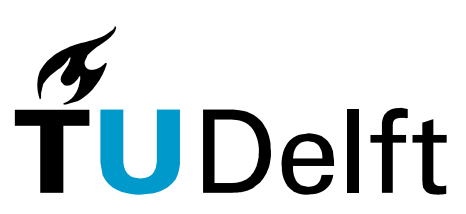

Delft University of Technology

Copyright (C) Aerospace Engineering, Delft University of Technology All rights reserved. 


\section{DELFT UNIVERSITY OF TECHNOLOGY DEPARTMENT OF AERODYNAMICS}

The undersigned hereby certify that they have read and recommend to the Faculty of Aerospace Engineering for acceptance the thesis entitled "Flow visualization and force measurements on a hovering flapping-wing MAV 'DelFly II'” by Kristien M.E. De Clercq in fulfillment of the requirements for the degree of Master of Science.

Dated: April 2, 2009

Examination committee:

prof. dr. ir. H. Bijl

dr. ir. B. W. van Oudheusden

ir. R. de Kat

ir. B.D.W. Remes 



\section{Preface}

The present work is the last run towards my graduation at the faculty of Aerospace Engineering at the Delft University of Technology. I have cursed, cried and laughed both during and after study time. But above all I have learnt and I am grateful for this complete experience.

First of all I would like to thank Hester Bijl and Bas van Oudheusden, for giving the opportunity to turn my interest for insect flight and DelFly into a fascinating thesis project and for the enthusiasm with which they have supported this project.

Many words of gratitude go to Roeland de Kat for introducing me to the miraculous world of PIV and for teaching me how to cope with the unpredictability of expected problems. Loads of thanks are given to Bart Remes for the inspiring discussions and the numerous emergency operations on the DelFly model.

I owe an applause to the complete MAVlab team: to Christophe for the smiles with which he answered my questions for "just another small adaptation" to the software application, to Guido for his brave attempts to understand my writings, to Dennis for the creative input and precise work on the experimental setup and to Rick for his curiosity and geniality towards my work.

A hug is sent to all of my friends: to the Dushi's for the necessary counterbalance to masculinity, to Ronald for cheering up the endless UB-days, to the guys and girls of the Vijfde for my overall introduction to the Dutch culture, to Bart for keeping me playful, to the dudes and chicks of Plankenkoorts for the best means of passing time, to Menno for helping me to lift de laatste loodjes, to Daniel for the lessons in astonishment and passion and last but not least to the guys of the basement for just being themselves.

A kiss belongs to my family: to Mama, Papa, Jopa, Lies en Gert-Jan for their unconditional love and to Moemoe and Bommama because I know they would be proud. Without your support, encouragement and understanding I wouldn't have come this far. 
Abstract

The development of micro aerial vehicles is motivated by the need for autonomous unmanned aerial vehicles to observe barely accessible areas. Insect flight has been a source of inspiration during the development of DelFly, a flapping wing MAV at Delft University of Technology. In order to further decrease the overall size of DelFly, preserving the excellent flight performance, a thorough understanding of the aerodynamics is necessary.

Compared to steady airfoils, flapping wings are able to generate high lift coefficients due to unsteady aerodynamics. It is expected that the occurrence of a leading edge vortex at wing rotation plays an important role in unsteady lift generation. Due to the biplane wing configuration of DelFly a wing-wing interaction, called the clap and peel mechanism, is caused. This mechanism is assumed to further enhance the lift generation by expelling air downwards, omitting the need for a start and stop vortex to be shed and strengthening the leading edge vortex. The influence of wing flexibility and the resulting wing deformation during isolated rotation, called the flex motion, supposedly postpone the shedding of the stopping vortex and strengthen the leading edge vortex.

The motivation of the current investigation is to visualize the flow field around a flapping wing MAV with proven flight performance. Complex wing kinematics, highly threedimensional flow and fluid-structure interaction are thus included without simplifications. Particle image velocimetry measurements and simultaneous force measurements are performed on the DelFly II flapping-wing MAV, to investigate the flow-field behaviour and the aerodynamic forces generated.

The high flexibility causes the wings to peel and flex during the rotation at the minimal and maximal amplitude respectively. This passive wing deformation largely influences the flow field behaviour.

The wings start peeling apart before the clap has been completed. During the peel, the PIV analysis shows a strong influx between the wings and a conical vortex structure above the leading edges. This peel mechanism contributes significantly to the lift, as revealed by the force measurements.

During the clap, the presence of a mirror wing causes the air to be expelled downwards and to roll up into vortices of the same direction as the bound vorticity of the subsequent stroke. The jet generated by the clap is stronger on the inner half of the wings, but exceptionally weak on the outer half. This is attributed to the high flexibility of the wing 
foil. The strong and chaotic starting vortex shed at the beginning of the outstroke, is responsible for a strong decline in lift force during the subsequent translational phase. The clap mechanism does rather attenuate than enhance lift.

The occurrence of a leading edge vortex during the flex can not be assessed from the PIV images due to optical obstruction, but is likely to appear since the wing flexing is accompanied with a large increase in lift. An additional augmentation in lift could also be attained by the delayed shedding of a combined starting and stopping vortex. Although this flap phase is accompanied by an increased lift, it does not contribute to the total lift as much as the peel mechanism. The PIV visualization first suggests the occurrence of a leading edge vortex at the onset of the subsequent translational stroke.

Especially during the flex the high deflection of the wings achieved at a higher flapping frequency is favourable. The force plot shows a relatively higher and broader peak in lift force. The aerodynamic mechanism underlying this phenomenon could not be identified. 
Table of contents

Preface

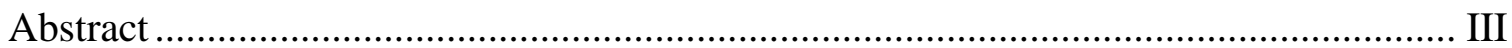

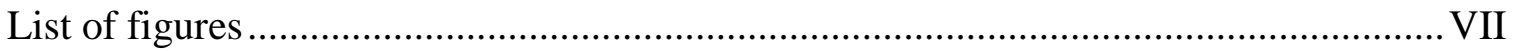

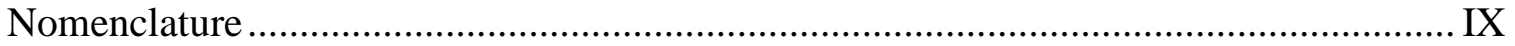

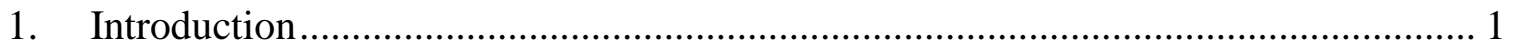

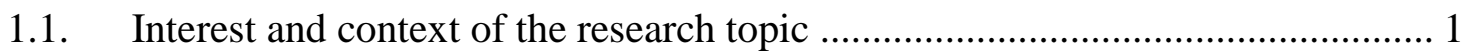

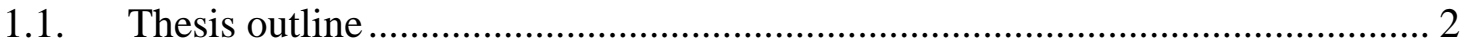

2. The flapping wing MAV "DelFly II" ........................................................... 5

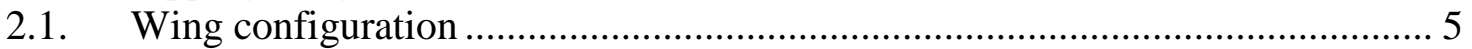

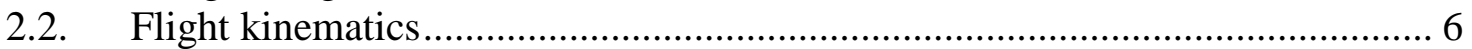

3. Unsteady aerodynamics in flapping flight ........................................................ 9

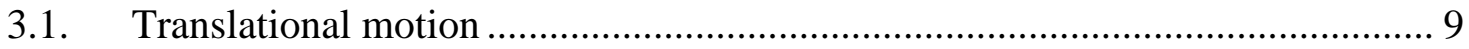

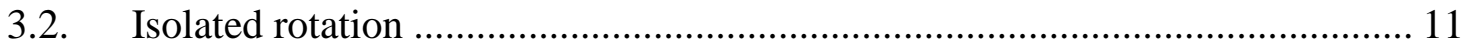

3.3. Clap and fling mechanism .................................................................... 12

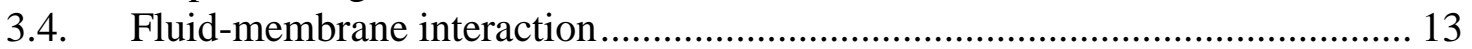

3.4.1. Flex mechanism during isolated rotation............................................ 14

3.4.2. Clap and peel mechanism ................................................................. 14

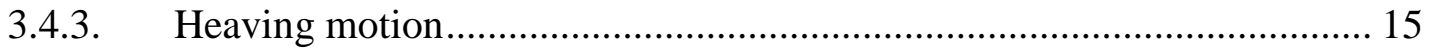

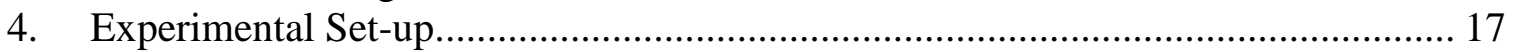

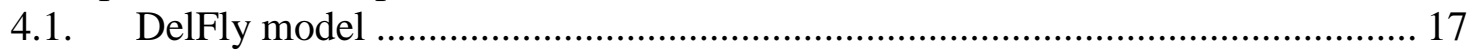

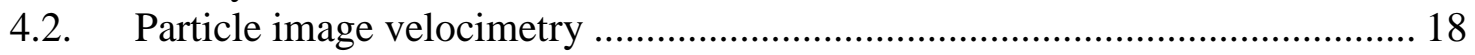

4.2.1. Working principle and advantages....................................................... 18

4.2.2. Measurement plane ........................................................................... 19

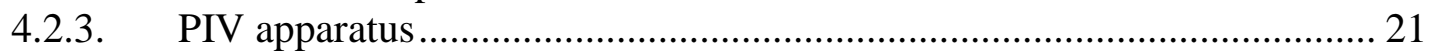

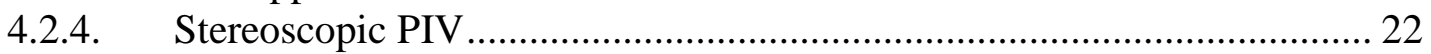

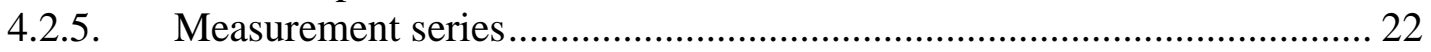

4.2.6. Image analysis............................................................................ 24

4.2.7. Vortex identification ..................................................................... 25

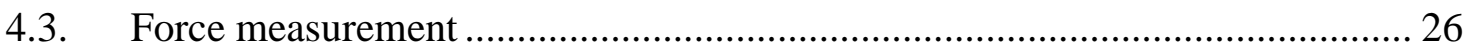

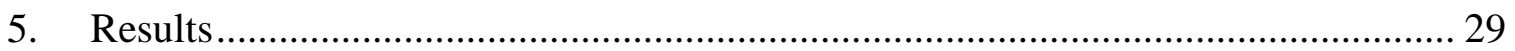

5.1. Observation of the wing motion and deformation .......................................... 29

5.2. Force measurements related to wing motion ................................................. 31

5.3. Fluid dynamics related to force generation...................................................... 32

5.3.1. Clap and peel mechanism ..................................................................... 34

5.3.2. Flex mechanism …………………………................................... 38

5.3.3. Influence of flap frequency .............................................................. 40

6. Conclusions and recommendations..................................................................... 43

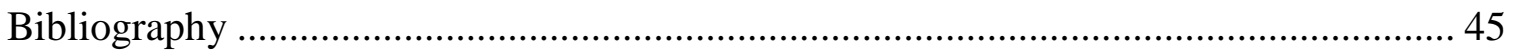


Appendix

A. Reynolds number of flapping flight ..................................................................... 47

B. V-component of time resolved PIV measurements for different time instants and

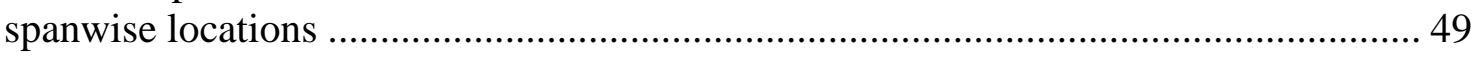

C. Vortex visualization of time resolved PIV measurements for different time instants

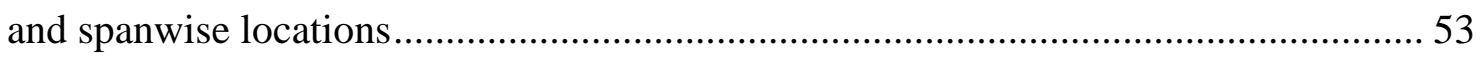

D. Vortex visualization of phase locked PIV measurements...................................... 57

E. V-component of time resolved PIV measurements zoomed in on clap and fling for different time instants and spanwise locations.............................................................. 58

F. Vortex visualization of time resolved PIV measurements in the wake for different

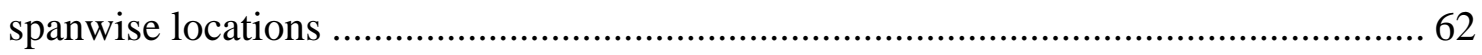




\section{List of figures}

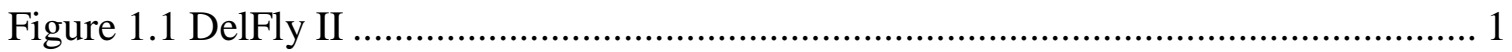

Figure 2.1 Geometric flapping parameters of DelFly ………………………….............. 5

Figure 2.3 Schematic representation of the fluid velocity with respect to the wing section

Figure 2.4 schematic representation of the wing path ............................................... 7

Figure 3.1 Schematic representation of the streamlines ............................................. 10

Figure 3.2 Flow visualization of the leading edge and tip vortex................................... 10

Figure 3.3 Schematic representation of the vortex structures around an impulsively

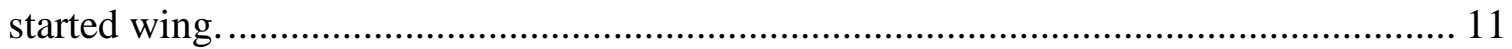

Figure 3.4 Schematic representation of the starting (yellow) and stopping (red) vortex sequence ............................................................................................................... 11

Figure 3.5 Schematic reconstruction of vortex generation and shedding during the clapand-fling maneuver ........................................................................................... 12

Figure 3.6 Schematic representation of the flex mechanism of a single wing ................ 13

Figure 3.7 Schematic representation of the clap and peel mechanism........................... 15

Figure 3.8 Pear-shaped wing tip trajectory …………….......................................... 15

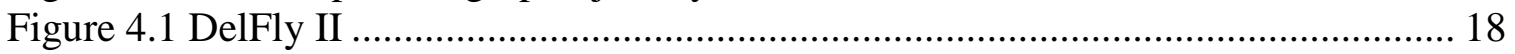

Figure 4.2 Overview of PIV measurement technique.................................................... 19

Figure 4.3 Schematic representation of the PIV apparatus........................................... 20

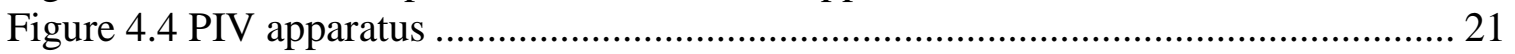

Figure 4.5 Different fields of view .............................................................................. 23

Figure 5.1 Schematic representation of the wing motion during one flap cycle .............. 30

Figure 5.2 Upward force fluctuations and flapping angle during one flap cycle ............. 31

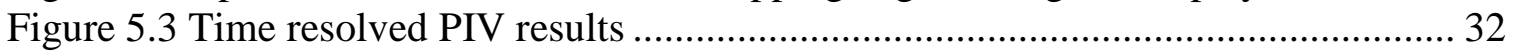

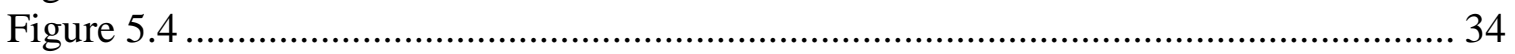

Figure 5.5 Vortex identification using $\lambda$-method at $\tau=0.11$ for $0.75 \mathrm{R}$ and $\mathrm{f}=13 \mathrm{~Hz} \ldots 35$

Figure 5.6 Spanwise variation in V-component of velocity during the clap .................... 36

Figure 5.7 Vortex identification using $\lambda$-method at $\tau=0.16$ for $0.75 \mathrm{R}$ and $\mathrm{f}=13 \mathrm{~Hz} \ldots .37$

Figure 5.8 Spanwise variation in V-compoment of velocity during the peel ................... 37

Figure 5.9 Vortex identification using $\lambda$-method at $\tau=0.26$ for $0.75 \mathrm{R}$ and $\mathrm{f}=13 \mathrm{~Hz} \ldots . .37$

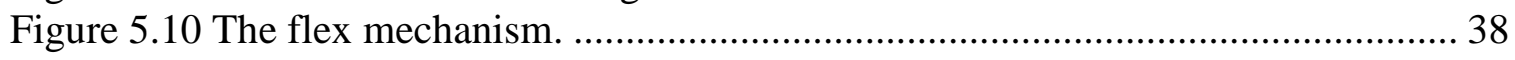

Figure 5.11 Spanwise variation in V-component of velocity during the flex................... 39

Figure 5.12 Spanwise variation in V-component of velocity during the instroke ............ 40

Figure 5.13 Phase locked PIV results ........................................................................... 41

Figure 5.14 Upward force fluctuations during one flap cycle ......................................... 42

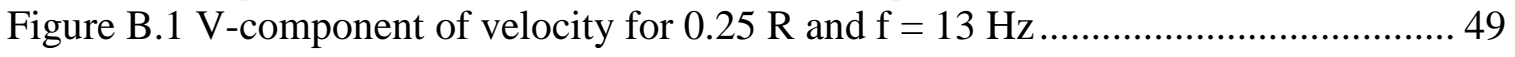

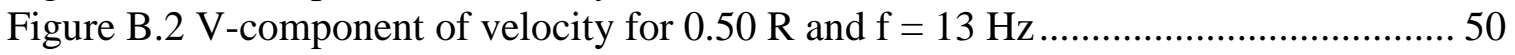

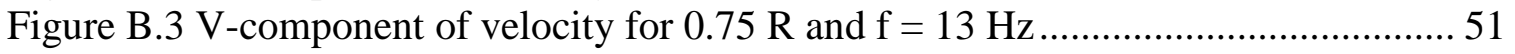




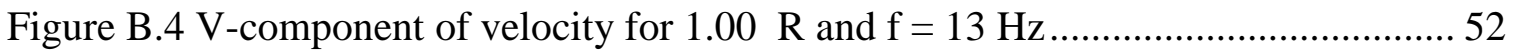

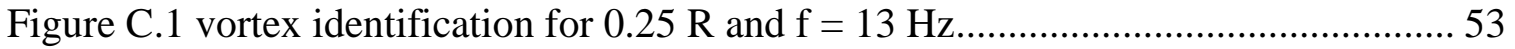

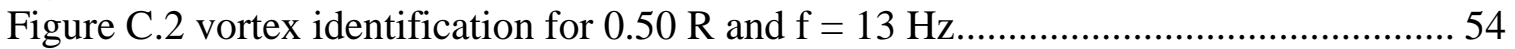

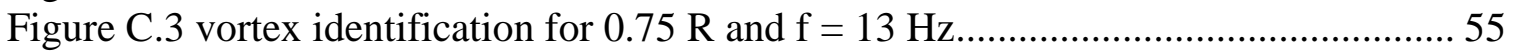

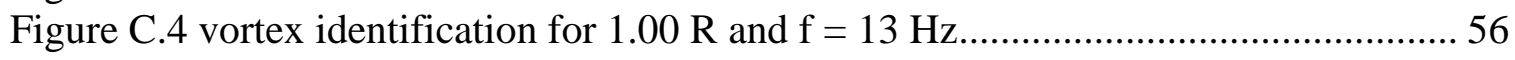

Figure D. 1 Vortex Identification for $0.75 \mathrm{R}$ and $\mathrm{f}=11 \mathrm{~Hz}$...................................... 57

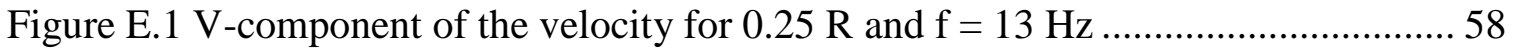

Figure E.2 V-component of the velocity for $0.50 \mathrm{R}$ and $\mathrm{f}=13 \mathrm{~Hz}$............................59

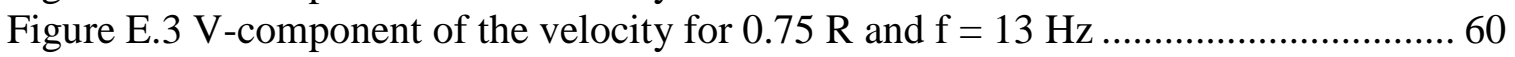

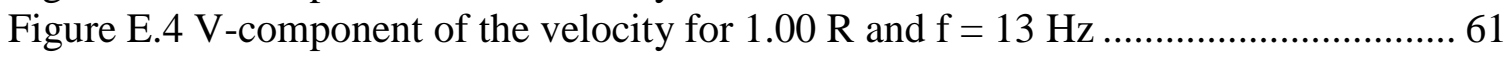

Figure F.1 vortex identification in the wake for $0.25 \mathrm{R}$ and $\mathrm{f}=13 \mathrm{~Hz} \ldots \ldots \ldots \ldots \ldots \ldots \ldots \ldots . . . . . . . . . . . .62$

Figure F.2 vortex identification in the wake for $0.50 \mathrm{R}$ and $\mathrm{f}=13 \mathrm{~Hz} \ldots \ldots \ldots \ldots \ldots \ldots \ldots \ldots . . . . . . . . . . . .63$

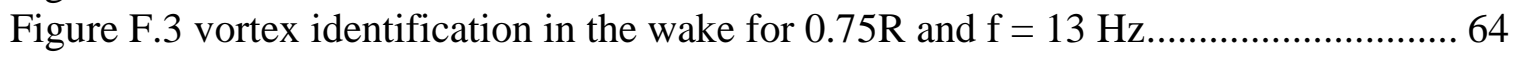

Figure F.4 vortex identification in the wake for $1.00 \mathrm{R}$ and $\mathrm{f}=13 \mathrm{~Hz} . \ldots \ldots \ldots \ldots \ldots \ldots \ldots \ldots . . . . . . . . . . . . .65$ 


\section{Latin symbols}

$\begin{array}{ll}\text { A } & \text { aspect ratio } \\ \text { b } & \text { wing semi span } \\ \text { c } & \text { chord length } \\ \text { D } & \text { aperture diameter } \\ \text { d } & \text { diameter } \\ \text { f } & \text { flapping frequency } \\ f & \text { focal length } \\ f_{\#} & \text { f-stop number } \\ \mathrm{g} & \text { gravitational force } \\ \mathrm{M} & \text { magnification factor } \\ \mathrm{p} & \text { pressure } \\ \mathrm{Q} & \text { second invariant } \\ \mathrm{R} & \text { wing length } \\ \mathrm{r} & \text { distance between wing tips } \\ \text { Re } & \text { Reynolds number } \\ \mathrm{S} & \text { shear strain rate } \\ \mathrm{S} & \text { wing planform area } \\ \mathrm{T} & \text { cycle period } \\ \mathrm{t} & \text { time } \\ \mathrm{u}, \mathrm{v}, \mathrm{w} & \text { velocity components in } \mathrm{x}, \mathrm{y}, \mathrm{z} \text { direction respectively } \\ \mathrm{V} & \text { vertical velocity } \\ \text { W } & \text { weight } \\ & \\ \text { Greek symbols } \\ \theta & \text { viewing angle } \\ \lambda & \text { eigenvalue } \\ v & \text { kinematic viscosity } \\ \xi & \text { vorticity } \\ \rho & \text { density } \\ \tau & \text { dimensionless time } \\ \varphi & \text { maximal stroke angle } \\ \Omega & \text { dihedral angle } \\ \Omega & \text { vorticity magnitude } \\ & \end{array}$




\section{Subscripts}

$\mathrm{S}$

clearance

ind induced

0 neutral

\section{Abbreviations}

PIV particle image velocimetry

TUDelft Delft University of Technology 


\section{Introduction}

\subsection{Interest and context of the research topic}

The development of micro aerial vehicles is motivated by the need for autonomous unmanned aerial vehicles to observe barely accessible areas, such as disaster or war areas, wildlife reserves, high constructions etc. These missions require a small and highly manoeuvrable airplane operating at low flight speed, as a stable camera platform and with the possibility of short landing and take-off ${ }^{1}$. In contrast to the usual fixed wings, rotary wings generate both lift and thrust resulting in a high manoeuvrability and low flight velocity. However the complexity of the construction limits the miniaturization of helicopters ${ }^{2}$. A solution to this problem has been found in nature. Flapping wings, as used by birds and insects, generate lift and thrust and subsequently introduce a favourable manoeuvrability and wide flight envelope. A fully controllable vehicle can be achieved with a relatively simple drive mechanism that offers the potential of minimization.

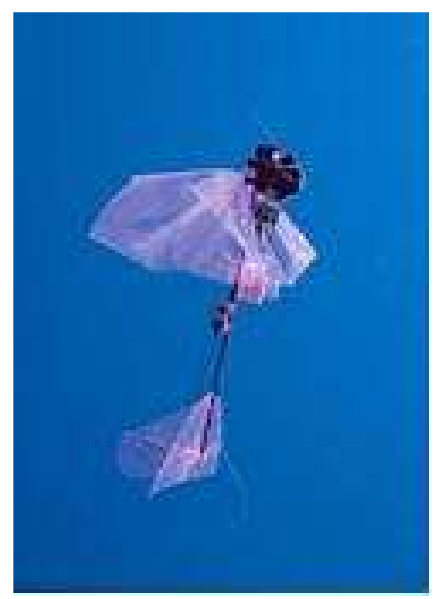

Figure 1.1 DelFly II

Insect flight has been a source of inspiration during the development of a series of flapping wing MAVs at Delft University of Technology (Figure 1.1). In the DelFly research project ${ }^{3}$, a top-down approach is used. Herein one starts with a fully functioning (relatively large-scale) ornithopter. By studying this ornithopter, theoretical insights can be gained into the necessary properties for a smaller version. Research then progresses by creating and studying ever smaller systems, while always maintaining a fully functioning flying robot. One advantage of this approach is that it allows interplay between theory and practice. 


\section{Introduction}

In order to further decrease the overall size of DelFly, preserving the excellent flight performance, a thorough understanding of the aerodynamics is necessary. Compared to steady airfoils, flapping wings are able to generate high lift coefficients due to unsteady aerodynamics. The lift enhancement has been attributed to specific flow structures that occur during translational and rotational motion. How these mechanisms manifest in reality has been the topic of experimental studies on a broad range of animal species. Experimental studies on insects are hampered by the small scales and high frequencies, whereas studies on scaled robotic models are limited to simplified simulations of the complex wing kinematics and deformations. Moreover, since DelFly II differs from typical insects and birds in wing configuration, flapping frequency and wing size, only a restricted similarity in flow-field behaviour is expected.

Numerical techniques to simulate the aerodynamics of flapping flight are being developed at the aerospace group of Delft University of Technology ${ }^{4,5,6}$. The analytical models are limited to simplifications of the complex wing kinematics, highly threedimensional flow and fluid-structure interaction. Therefore the numerical simulations are currently insufficient for the prediction of the flow field around DelFly and cannot be directly applied to the development of DelFly.

The motivation of the current investigation is to visualize the flow field around a flapping wing MAV with proven flight performance. Simplifications to the complex interaction of several effects active in the aerodynamics of flapping wings are avoided in this manner. A full-scale and non-simplified model of DelFly II is considered. The flow field around the wings was analyzed by particle image velocimetry (PIV) while simultaneous force measurements indicate the contribution of visualized flow structures to the lift generation.

The experience in experimental aerodynamics on a flexible flapping wing model, gained in this joint project between the Aerodynamics Department and the MAV-lab of TUDelft, will serve as a guideline for the formulation of future research goals and the design of further experimental campaigns.

The data obtained in this study will furnish the possibility to validate numerical codes and will be evaluated to contribute to the improvement of the aerodynamic characteristics of DelFly II.

\subsection{Thesis outline}

In chapter 2 the particular wing configuration and kinematics defining the aerodynamic characteristics of DelFly II are introduced. A theoretical flow analysis is applied to the typical wing motion and illustrated by observations on insects in chapter 3. Distinction is made between three different phases of the flapping motion: the translational phase, the isolated rotation and the clap- and fling-motion. Thereafter the influence of the wing flexibility on these phenomena is outlined. The level of agreement between the theoretical and actual flow field has been examined in an experimental campaign. The 


\subsection{Thesis outline}

experimental set-up will be described in chapter 4 . This chapter contains details about the flapping wing model, the PIV system and the force measurement apparatus. The analysis of the results is represented in chapter 5, referring to the distinct phenomena introduced in chapter 3 . This leads to the conclusion and recommendations for further investigation in chapter 6 . 
1 Introduction 


\section{The flapping wing MAV "DelFly II"}

In the course of a systematic technical design the most favourable concepts of biological flight were combined and adapted to the specific requirements of the design of DelFly. The result is an MAV with flexible flapping wings in biplane configuration (Figure 1.1). One of the most determining features of DelFly's appearance is its wing configuration. The choice for this particular configuration, discussed in section 2.1, largely influences the aerodynamics and is crucial for the flight performance.

DelFly II is the world's first ornithopter to attain an exceptionally broad flight envelope with the same configuration. Different throttle and elevator commands result in entirely different flight behaviors. The DelFly is not only capable to fly with a maximum forward speed of $7 \mathrm{~m} / \mathrm{s}$ and to hover; it can even fly backwards with a speed up to $1 \mathrm{~m} / \mathrm{s}$. An analysis of the typical wing motion and the aerodynamic forces explains in section 2.2 how such a broad flight envelope is possible.

\subsection{Wing configuration}

With the aim to create a stable camera platform, DelFly has been designed with two pairs of wings flapping in anti-phase. To maximize the lifting surface within the stringent size limitations, both wings are positioned on top of each other (Figure 2.1). To obtain lateral stability in forward flight, the wings are positioned symmetrically with respect to the fuselage plane at a dihedral angle $\psi$. To prevent impact damage from clapping, a small clearance $\left(\varphi_{s}\right)$ is provided between the upper and lower wing in their neutral position.

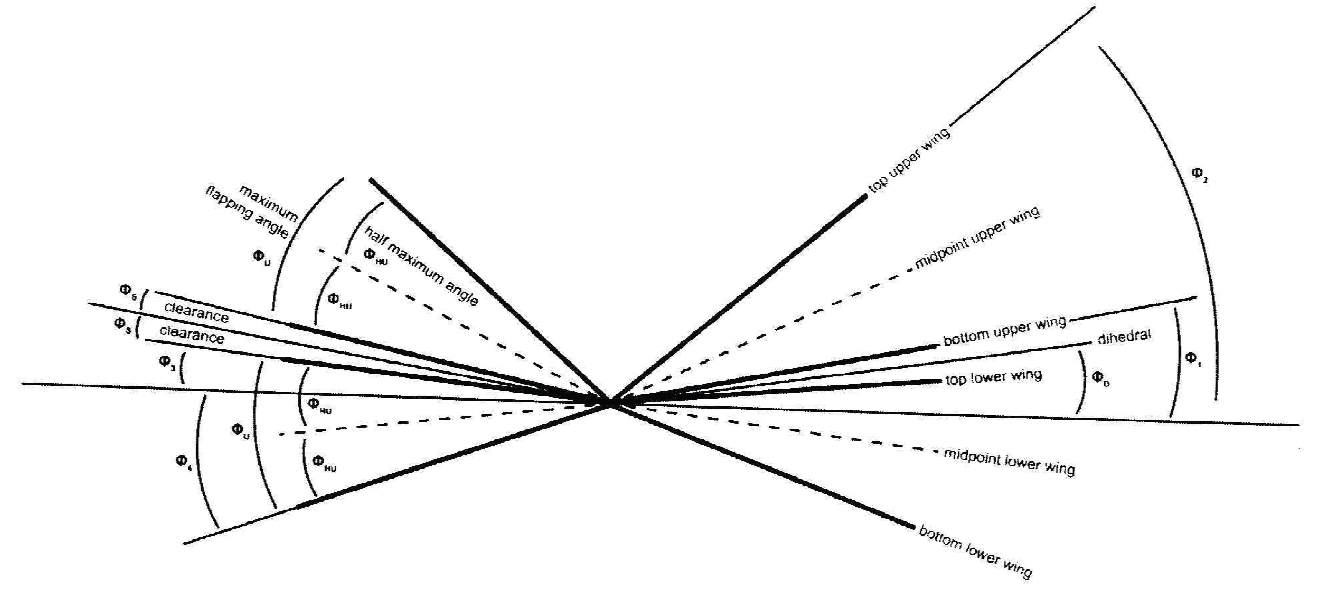

Figure 2.1 Geometric flapping parameters of DelFly. Indicated are the stroke angle $\varphi$, the dihedral angle $\psi$ and the clearance angle $\varphi_{s}$ 
Hinged in one point and controlled by a crank-shaft mechanism moving the leading edges, the wings move to and fro in a single plane. This motion will be termed the translational motion. Under combined inertial and aerodynamic loading the wings rotate between subsequent strokes, called the rotational motion, such that the anatomical leading edge always leads. Due to inertia and elasticity the wings are dynamically twisted. The upper and lower wings flap at the same frequency and over an equal stroke angle $\varphi$. The optimal flap angle and frequency for hovering, maximizing thrust for minimal power consumption, has been investigated experimentally by N. Bradshaw ${ }^{7}$. To maximize the payload capacity, the flap angle is maximized within the limitations of the drive mechanism.

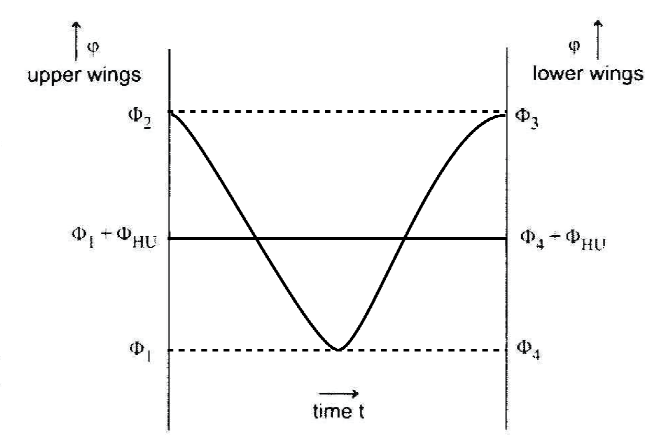

Figure 2.2 Flap angle variation during one flapping sequence

\subsection{Flight kinematics}

To explain the exceptional flight envelope of DelFly II we look at a simplified analysis of the typical wing motion.

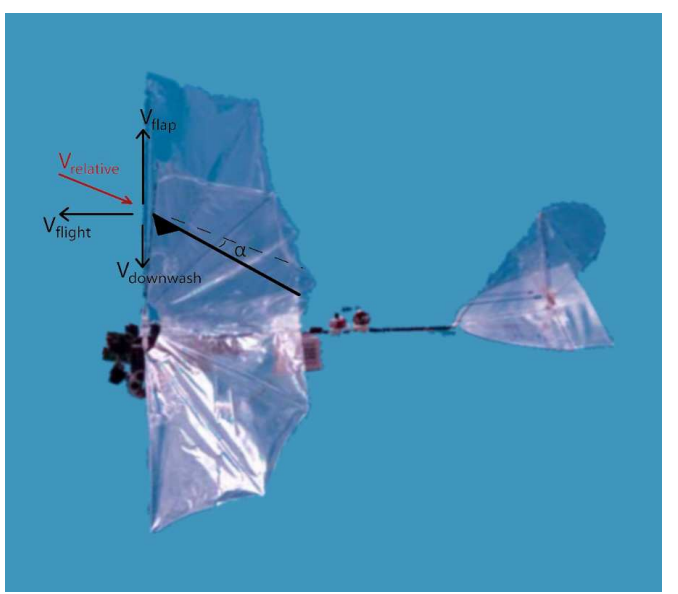

Figure 2.3 Schematic representation of the fluid velocity with respect to the wing section The morphological lower surface is indicated by the triangle at the leading edge of the outlined wing section. 
The velocity of the wing with respect to the air (Figure 2.3), defined by the flight, flap and downwash velocity, is tangent to the wing path. Accordingly the lift and drag force are respectively perpendicular and tangent to the wing path. The wing path during fast forward flight is shown in Figure 2.4a. The stroke plane is positioned vertically and the fuselage horizontally, minimizing the drag. The tail is used as in a normal plane configuration.

By pulling the elevator up, the body tilts nose-up and the flight speed decreases, until the stroke plane becomes horizontal during hovering. Figure $2.4 \mathrm{~b}$ shows the wing path and related forces for hovering flight. The effective velocity is now only defined by the flap and downwash velocity. The motion of the upper and lower wing pairs is symmetric, providing weight support but cancelling the horizontal thrust. The reader should notice that the anatomical lower wing surface, indicated by the triangle, does not act as the aerodynamic lower surface during the complete flap cycle.
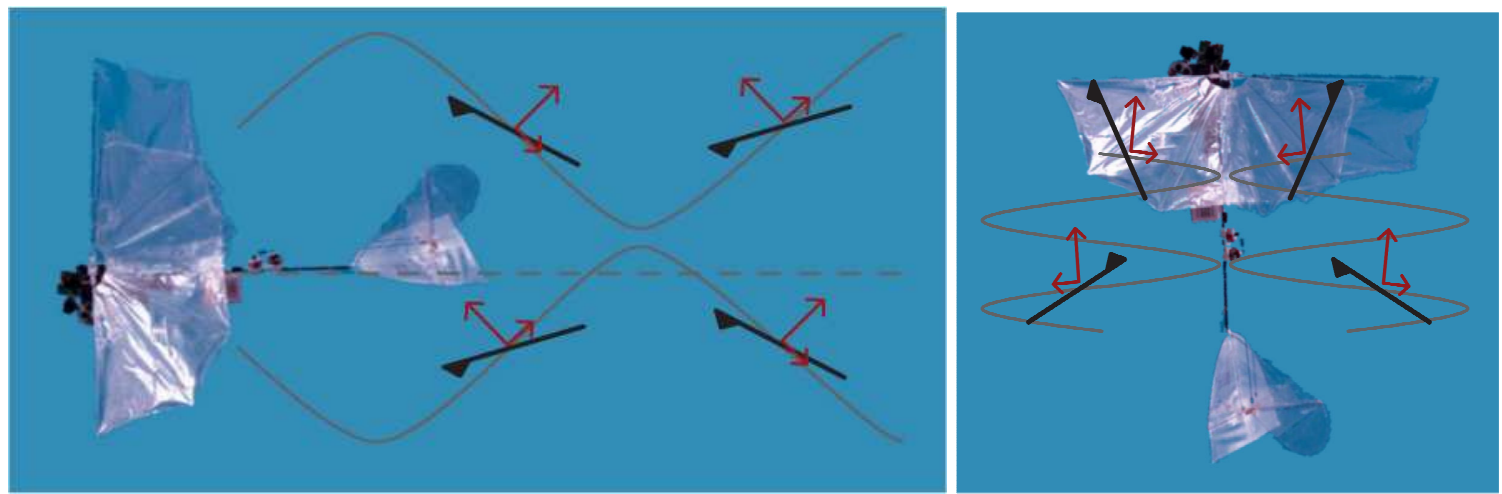

Figure 2.4 schematic representation of the wing path for a DelFly in a) forward flight and $b)$ hovering. The morphological lower surface is indicated by the triangle at the leading edge of the outlined wing section. The resultant aerodynamic forces for both strokes are shows. 
2 The flapping wing MAV "DelFly II" 


\section{Unsteady aerodynamics in flapping flight}

To provide a basis for the analysis of the experimental data, first some theoretical aerodynamical concepts are outlined in this chapter. Compared to steady airfoils, flapping wings are able to generate high lift coefficients due to unsteady aerodynamics. The lift enhancement has been attributed to specific flow structures that occur during the different phases of the flap cycle ${ }^{8}$. Below, the role of these effects is theoretically discussed and illustrated by observations on animals. Distinction is made between the translational and isolated rotational motion in section 3.1 and 3.2 respectively. The influence of a mirror wing in close proximity during rotation is considered in section 3.3. Initially, the wing is assumed rigid, such that the wing attitude and shape do not change during the course of a wing beat. The influence of wing flexibility and the resulting wing deformation during rotation is treated subsequently in section 3.4.

\subsection{Translational motion}

The wings move to and fro in a single plane. At the beginning of a stroke, the wing is impulsively started. To visualize the different mechanisms, instantaneous streamlines are used. Air swirls around the sharp trailing edge introducing a region of intense vorticity (Figure 3.1b). Kelvin's circulation ${ }^{9}$ theorem states that the time rate of change of circulation around a closed curve consisting of the same fluid elements is zero. Therefore a vortex, equal in strength but opposite in direction to the trailing edge vortex, is generated around the airfoil. The high-vorticity region moves downstream of the trailing edge and tends to roll up into a concentrated starting vortex. As long as vorticity is shed from the trailing edge, the starting vortex grows stronger and so does the bound vortex, until the flow will conform to the Kutta condition' (Figure 3.1a). This gradual build-up of circulation is called the Wagner effect ${ }^{10}$.

Since the strength of the bound vortex is directly proportional to the velocity difference and hence the pressure difference over the airfoil section, the build-up of vorticity is a measure of the increase in lift force generated per unit span. The total distance travelled by the wings during a half stroke is small, thus the circulation growing in a manner similar to Wagner's theory will remain lower than the steady value. Generally, the required lift to support body weight in flapping flight is larger than the lift producible by the wings in steady-motion. A source of additional lift has to be found. 


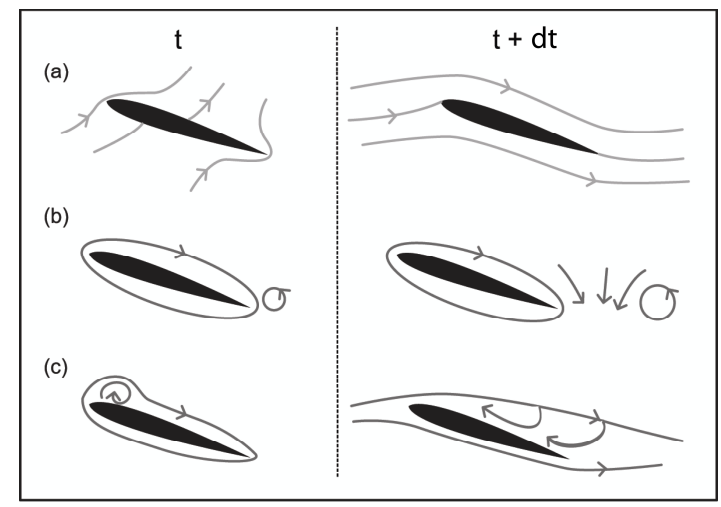

Figure 3.1 Schematic representation of the streamlines formed due to a) Kutta condition; b) Kelvin's theorem and c) dynamic stall

When the wings are impulsively started at an angle of attack higher than the stall angle, a phenomenon called dynamic or delayed stall ${ }^{12}$ is expected. The wing travels several chord lengths at large incidences before the separation associated with stall becomes well-developed. The flow separates from the wing surface as it swirls around the sharp leading edge, mixes with the surrounding air and re-attaches to the downstream wing surface (Figure 3.1c). The separation bubble is called the leading edge vortex. During the brief period between $t$ and $t+d t$, the circulation, and thus the lift, exceeds the maximum quasi-steady stalled value. The circulation starts to grow at the beginning of the translational stroke and is proportional to the product of the chord and the span wise location. The steady-state stall will not be reached if the stroke length covers only a few chords, as is the case for flapping wings.

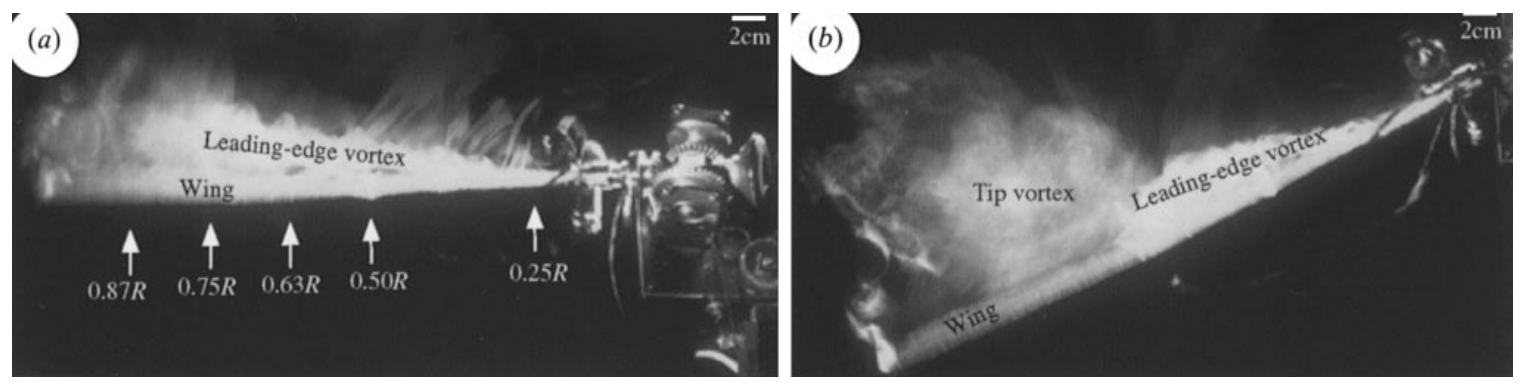

Figure 3.2 Flow visualization of the leading edge and tip vortex on robotic model of Manduca by van den Berg et al ${ }^{14}$.

How these mechanisms manifest themselves in reality has been the topic of experimental studies on a broad range of species. Especially interesting in the scope of this study are the results of research on the hawkmoth Manduca Sexta, which operates in a Reynolds range similar to DelFly II (Appendix A). Alike DelFly, the hawkmoth tilts its stroke plane horizontally to hover. The studies by Ellington's group ${ }^{13,14}$ confirmed the existence of a leading edge vortex formed above the wing during downstroke (Figure 3.2). The work of van den Berg ${ }^{14}$ indicates that the dynamic stall mechanism is clearly responsible for the leading edge vortex generation, since the circulation builds up during the translational stroke. The vortex increases in size and strength along the wingspan proportional to the product of the chord and the span wise location. This implies that the 
vortex grows as a conical spiral and that no vortical structure is present between the wing bases.

\subsection{Isolated rotation}

When the translational wing motion stops at the end of the stroke, no lift or circulation should be left. The bound vortex swirls off the trailing edge, shedding as a stopping vortex (Figure 3.3). In the same way as circulation grows at the beginning of a half stroke due to the Wagner effect, it decays gradually at the end. The decaying vorticity will interfere with the build up of circulation on the following half stroke, which is in the opposite direction (Figure 3.4).

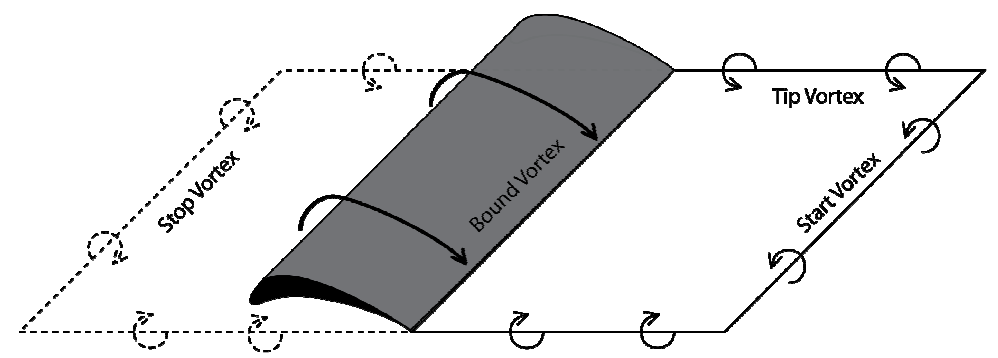

Figure 3.3 Schematic representation of the vortex structures around an impulsively started wing. Dashed lines and vortices indicate future events ${ }^{\mathbf{8}}$.

In hovering flight, due to the absence of flight velocity, the shed vorticity moves slowly down into the wake. The stopping vortex of one stroke has the same direction as the starting vortex of the next. These vortices may shed together, possibly hampering the formation of the new stroke's bound vortex, what will reduce the lift. To prevent this, a more efficient rotational mechanism should be used, as is discussed in the next section.

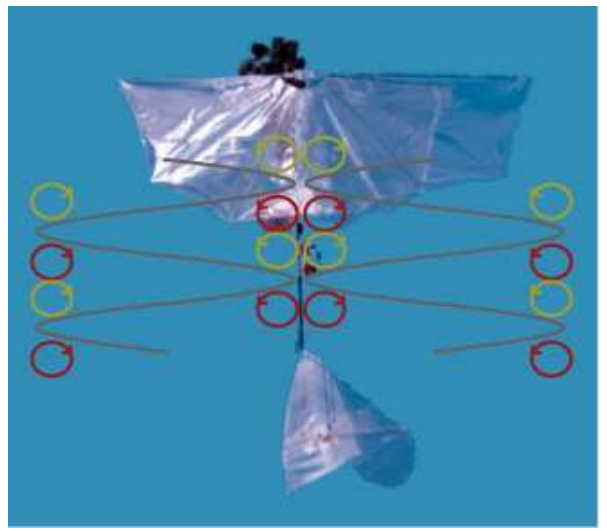

Figure 3.4 Schematic representation of the starting (yellow) and stopping (red) vortex sequence 
At the wing tips, the flow is forced from the high-pressure region underneath the foil to the low-pressure region on the top, forming the tip vortices. These tip vortices in combination with the starting and stopping vortices form a closed quasi-rectangular vortex ring (Figure 3.3).

\subsection{Clap and fling mechanism}

As a result of the positioning of the wings on top of each other, both wings come together near the neutral position and an additional lift generation mechanism is supplied.

Near minimal stroke angle $\left(\varphi_{1}\right.$ and $\varphi_{3}$ in Figure 2.1), the angles of attack increase as the wings decelerate and the upper and lower wings touch along their surfaces. By expelling air downwards, this clap mechanism ${ }^{17}$ may produce a momentum jet augmenting lift. Otherwise the lift enhancement could be interpreted as a method to extend lift generation till the clap is completed. Since the bound vorticity of both wings is equal in strength but opposite in sign, Kelvin's theorem applies without the need for a stopping vortex to be shed.

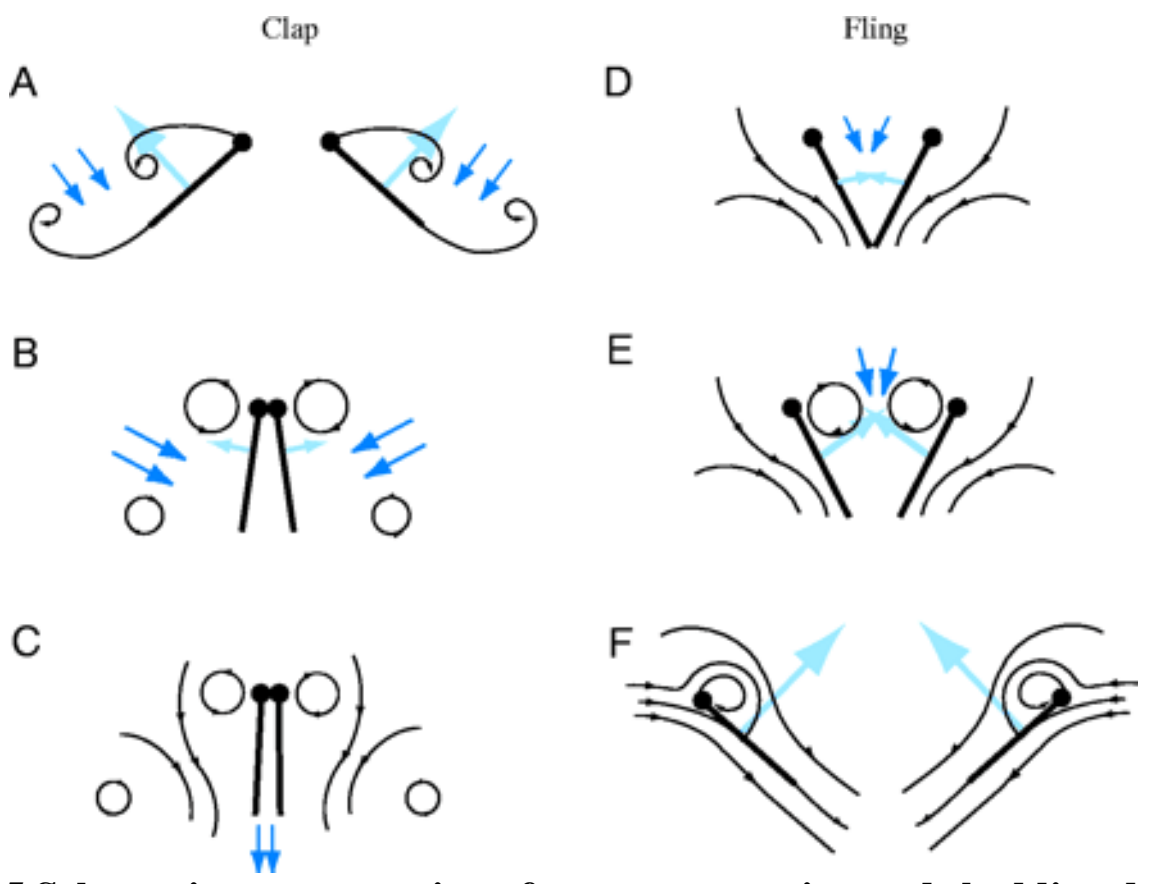

Figure 3.5 Schematic reconstruction of vortex generation and shedding during the clap-and-fling maneuver as described by Weis-Fogh; black lines show streamlines, dark blue induced velocity and light blue net forces (from Sane ${ }^{15}$ )

In the second half of the rotation, the wings separate again. During this so-called fling motion $^{10}$, air flows into the low pressure region between the wings. The inflow of air generates a circulation of opposite direction around both wings. No starting vortices would be shed, since the bound vortex of one wing acts as the starting vortex of the other 


\subsection{Fluid-membrane interaction}

one. As the wings separate and move apart in the next stroke, enhanced lift would immediately be generated from the fling circulation, circumventing the gradual growth of circulation by the Wagner effect.

Due to the strong acceleration of the flow around the leading edge a leading edge vortex is supposed to be generated, which increases the net circulation around the wing. The strength of this vortex will grow with the opening angle, as vorticity shed from the leading edge accumulates during the fling. The vortex might become unstable and shed before the subsequent stroke.

Examples of insects using clap and fling as lift enhancement mechanism typically operate in a lower Reynolds range compared to DelFly II. On a dynamically scaled robotic model of a fruit fly, Lehmann ${ }^{16}$ recently investigated lift enhancement due to the fling mechanism. The clap mechanism was found to attenuate lift force. On the same model, Poelma ${ }^{18}$ demonstrated the existence of leading and trailing edge vortices.

\subsection{Fluid-membrane interaction}

Opposite to what is assumed in the previous analysis, DelFly's wings are not rigid. The wing surfaces, constructed of Mylar foil with carbon stiffeners (4.1), are highly flexible. Due to the flexibility and under the influence of both inertial and aerodynamic loading ${ }^{7}$ the wings profile changes continuously during a wingbeat sequence. This passive wing deformation, typical for the DelFly, is barely studied on insects and occasionally mentioned in literature. The fluid membrane interaction is expected to introduce some interesting aerodynamic features as will be analyzed in this section.

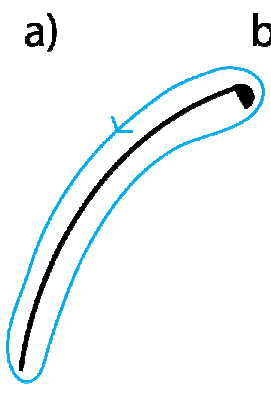

b)

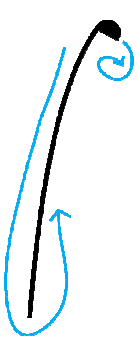

c)

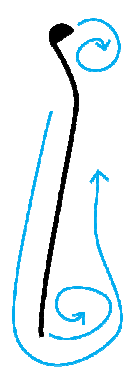

d)

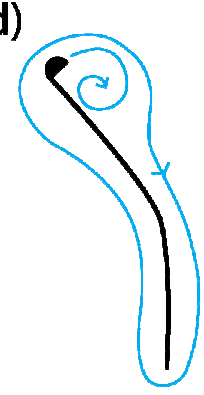

e)

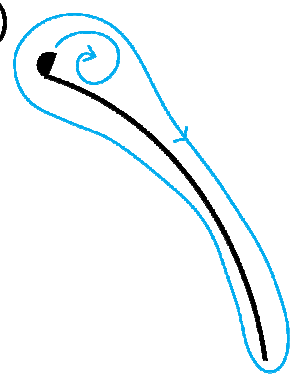

$(6)$

Figure 3.6 Schematic representation of the flex mechanism of a single wing. The semicircle on the outlined wing section is indicating the location of the leading edge. The blue lines represent the streamlines.

Wing flexibility plays an important role in flapping wings, especially during rotation. The changes in wing attitude during a flap cycle have been determined by visual inspection of high speed camera images of a hovering DelFly $\mathrm{II}^{7}$. The potential contribution of the wing deformation to the lift enhancement is analyzed in this section. The rotational 
3 Unsteady aerodynamics in flapping flight

mechanisms involved with a single wing and with the interaction between two mirror wings are treated separately in subsection 4.1 and 4.2 respectively.

\subsubsection{Flex mechanism during isolated rotation}

As the wings decelerate near maximal stroke angle, the angles of attack increase until the wing is perpendicular to the stroke (Figure 3.6a-b). Near the middle of rotation (Figure 3.6c-d) the wing flexes: the leading edge accelerates to the opposite direction, forcing the upper part of the wing to rotate, while the trailing edge stays almost stationary. Due to this flex mechanism ${ }^{19}$ the leading edge has a larger velocity and vorticity may shed into a separation vortex at the leading edge instead of the conventional starting vortex shed from the trailing edge, as discussed in section 3.2. This leading edge vortex is of the correct sense for the following half-stroke and stays attached to the wing at the beginning of translation. The leading edge accelerates at the beginning of the next half stroke and the wing unflexes (Figure 3.6e). The bound vorticity is likely to roll up around the stationary trailing edge and shed as a combined starting and stopping vortex when the wing unflexes. This mechanism would provide the wing with a net circulation at the beginning of the following half stroke, which skips the gradual increase of lift.

Smoke visualization with tethered Manduca Sexta moths ${ }^{13}$ investigated the use of the flex mechanism at the end of the downstroke. At the moment that the leading edge starts to rotate, the fore- and hind-wings flex. The increase in the velocity at the leading edge induces vortex shedding. This new leading edge vortex is in the correct sense as to enhance the bound circulation during the subsequent stroke. However, it does not remain attached to the wing.

\subsubsection{Clap and peel mechanism}

Instead of rigidly clapping together as previously assumed in section 3.3, the wings curve along their camber and the meeting point smoothly moves along the wing surfaces from the leading edge towards the trailing edges, as sketched in Figure 3.7a-d. Lift will be enhanced as for the clap previously described.

The leading edges start separating again, the wings curve toward the opposite side and the surfaces peel apart, as can be seen in Figure 3.7e-f. Due to this peel the circulation is expected to grow, instead of decay as in the fling, as the distance from leading edge to separation point 8 increases. As in the fling a leading edge vortex is generated. However, the vorticity is expected to be evenly distributed along the lengthening surface instead of rolling up into a large bubble. In this way the peel overcomes the problem involved with the Wagner effect as does the fling, but without the formation of a marginally stable leading edge vortex. 


\subsection{Fluid-membrane interaction}
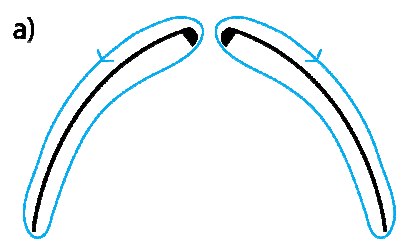

d)

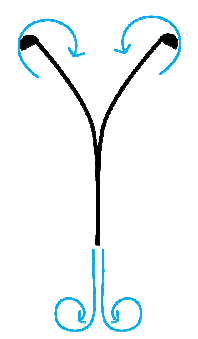

b)

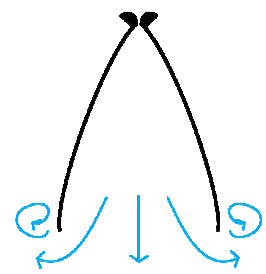

e)

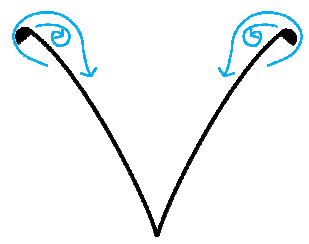

c)
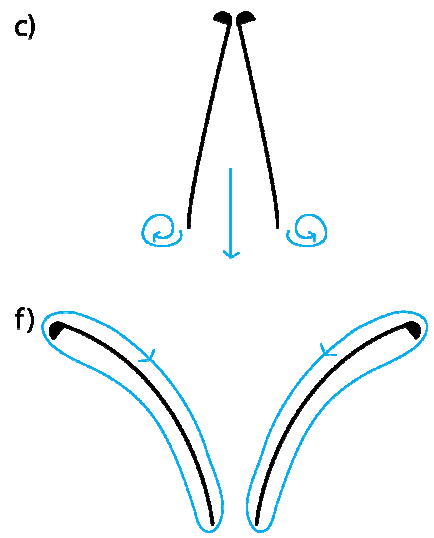

Figure 3.7 Schematic representation of the clap and peel mechanism. The semi-circle on the outlined wing section is indicating the location of the leading edge. The blue lines represent the streamlines.

On free-flying Butterflies, the peel mechanism has been observed to generate a fullyformed leading edge vortex as soon as the wings separate ${ }^{20}$. However, not any substantial change in size or form during the subsequent stroke has been visualized.

\subsubsection{Heaving motion}

Preliminary tests on the DelFly II showed that it was not able to fly with round crosssection carbon rods as leading edges. When cut in half, the resulting D-shaped carbon rods (Figure 3.6 and Figure 3.7) did not only allow the DelFly II to fly forward, but also to hover. The essential difference with the circular rod is a lower stiffness in the direction perpendicular to the stroke plane. It has been assumed that the wings make a heaving motion perpendicular to the stroke plane, which might contribute to a better aerodynamic performance. Though various patterns of heaving motion, for example the pear shaped trajectory (Figure 3.8), have been observed on insects21, the influence of this motion on the lift generation is not yet understood.

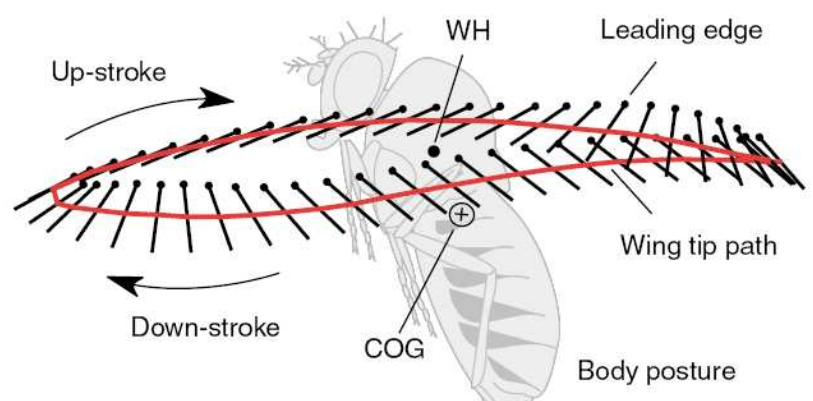

Figure 3.8 Pear-shaped wing tip trajectory of fruit fly by Lehmann et al. ${ }^{21}$ 
3 Unsteady aerodynamics in flapping flight 


\section{Experimental Set-up}

DelFly II differs in Reynolds-number (Appendix A), wing kinematics and flight performance from the insects studied by biologists and in complexity from the systems considered in other experimental and numerical studies. Therefore only a restricted similarity in flow field behavior is expected. The flow-field around a hovering DelFly is examined in an experimental campaign. Acquaintance of the similarities and discrepancies between the theoretical and actual flow field forms a basis for further improvement of DelFly's flight performance.

A full-scale and non-simplified model of a flapping wing micro aerial vehicle with proven flight performance, DelFly II, is considered and will be described in more detail in section 4.1. The flow field around the wings was analyzed by particle image velocimetry. The working principles of PIV and the experimental apparatus are described in section 4.2. Both time resolved and phase locked measurement series have been performed with a high speed stereoscopic PIV-system. Simultaneous force measurements provide information that may assist to interprete the contribution of visualized flow structures to the lift generation. The specifications of the force sensors used can be found in section 4.3.

\subsection{DelFly model}

The choice has been made to observe DelFly in hovering flight, since the hovering flight modus is the least efficient one and therefore necessitates the most improvement in the design. Moreover, the unsteady aerodynamic effects, which are hardly predictable but very interesting for lift enhancement, are assumed to dominate over the quasi-steady ones in hovering flight configuration.

Experiments are performed on full scale DelFly II-wings. The wings and gearbox planform are integrated in the experimental set-up without the usual fuselage and tail. The wings are positioned symmetrically with respect to the fuselage plane at a dihedral angle $\psi$ of $12^{\circ}$ (Figure 2.1). The wing semispan from root to tip is $14 \mathrm{~cm}$ (Figure 4.1 ) and the mean chord length, the ratio of the wing surface to the wing span, is $7.3 \mathrm{~cm}$ (Appendix A). The flapping motion is controlled by a crank-shaft mechanism moving the leading edges, hinged in one point, in a single plane. The maximal stroke angle $\varphi$ is $48^{\circ}$.

The leading edges are constructed of D-shaped carbon rods with a diameter of $0.7 \mathrm{~mm}$ and are relatively stiff in comparison to the extremely flexible wing surface. The wing surface is constructed of a $5 \mu \mathrm{m}$ tick Mylar foil, which has a E-modulus of 5GPa, a yield 


\section{Experimental Set-up}

strength of $145 \mathrm{Mpa}$ and a tensile strength of $214 \mathrm{MPa}^{22}$, stiffened with carbon bars of $0.28 \mathrm{~mm}$ diameter. Near the root the wing surface is attached to the body along the chord.

The wings were positioned with the stroke plane horizontally to simulate the hovering flight modus. To decrease environmental influences and wall interference the experiments were performed in a large cubic test volume of $3 \mathrm{~m} \times 3 \mathrm{~m} \times 3 \mathrm{~m}$, where the surrounding flow was stationary.
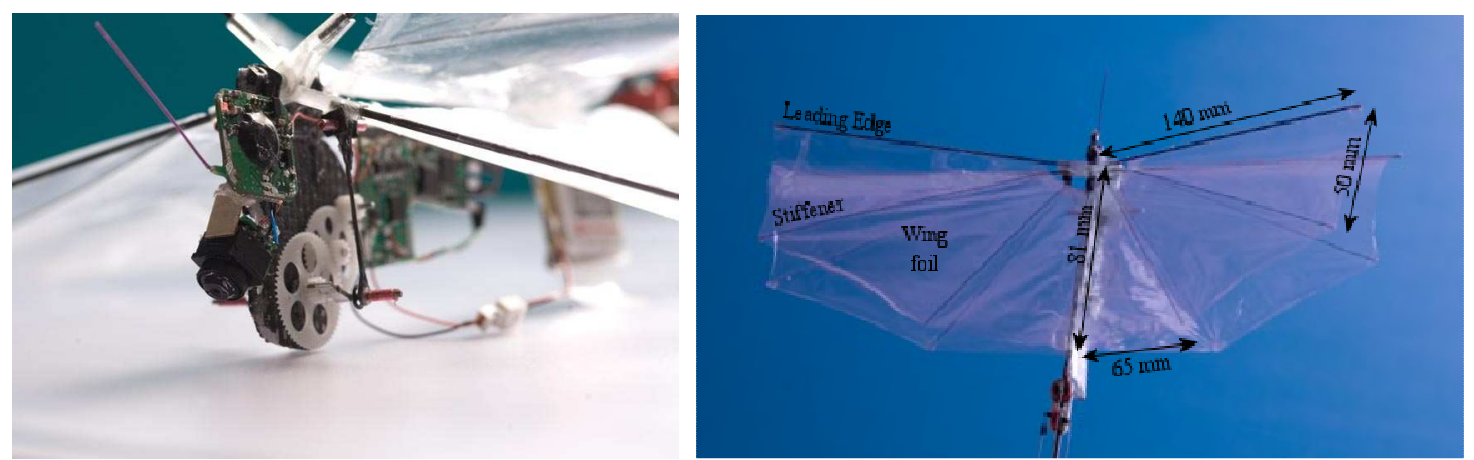

Figure 4.1 DelFly II $a$ ) the crank mechanism $b$ ) the wing planform dimensions

The mean wing beat frequencies were selected as 13 and $11 \mathrm{~Hz}$ for the time resolved and phase locked measurements respectively, which resemble typical flapping frequencies necessary to sustain hovering flight.

Regarding the wings stroke as an actuator disc of $0.03 \mathrm{~m}^{2}$ the flow velocity in chord wise direction has been defined as half the ratio of the generated force and the mass flux, corresponding to the actuator disc theory. This induced velocity of $1.5 \mathrm{~m} / \mathrm{s}$ was selected as the characteristic velocity. The Reynolds number based on the mean chord length and the induced velocity thus obtained is 7380 (Appendix A).

\subsection{Particle image velocimetry}

Particle image velocimetry has been used to visualize the flow field around the flapping wings. Before describing the features of the experimental setup into detail, the general concept will be briefly described in section 4.2.1 to support the understanding of the chosen parameters in section 4.2.3 which involve each other in a complex manner. For a more detailed review of this measurement technique is referred to the book by $\mathrm{Raffel}^{23}$.

\subsubsection{Working principle and advantages}

The principle of PIV is based on determining the velocity field of a fluid or gas flow by tracing the motion of particles immerged in the flow. It is assumed that the particles move with the flow, without changing it. Typically the particles in a planar section of the flow 
are illuminated twice in a short time interval by a laser light sheet. The light scattered by the particles is captured by a lens onto a CCD-chip of a camera as a sequence of frames.

For evaluation, the PIV recording is divided into small subareas called interrogation areas. The local average displacement between two illuminations is determined by crosscorrelation of the light intensity distribution over this interrogation window in two subsequent recordings. The maximum correlation value indicates the most probable particle displacement in that region. Taking into account the time-delay between two illuminations and the magnification at imaging, the local flow velocity vector is calculated. This process is repeated for all interrogation areas, yielding a velocity vector map of the imaged flow region.

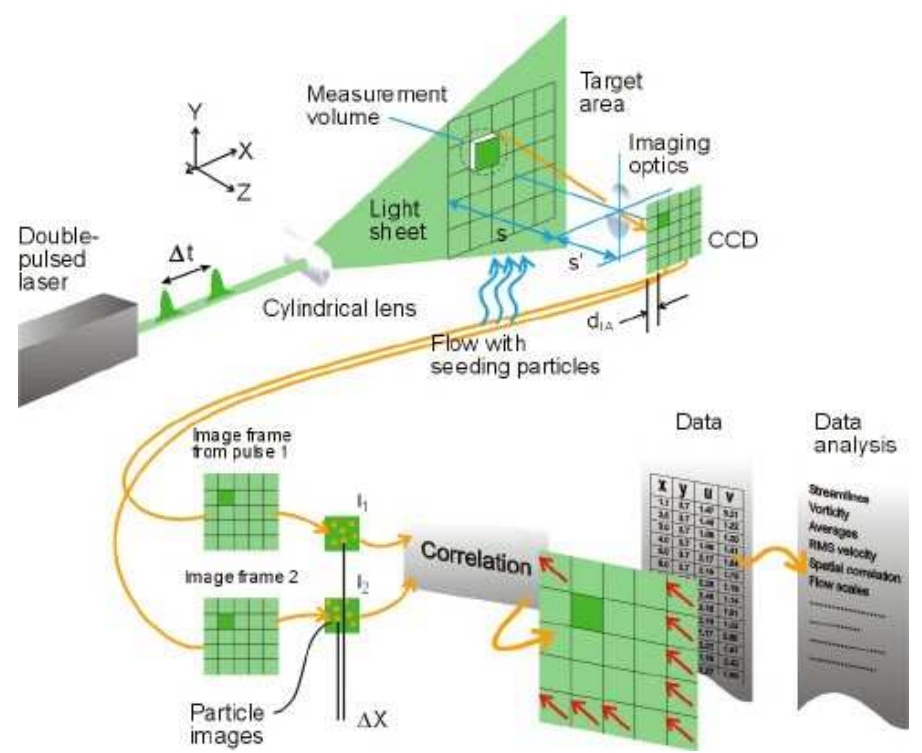

Figure 4.2 Overview of PIV measurement technique (from Dantec Dynamics ${ }^{24}$ )

The main advantage of this measurement technique is that it is non-intrusive. Assuming the use of suitable seeding, the optical measurement technique does not disturb the flow field. Additionally, PIV allows the instantaneous measurement of large parts of the flow field. In comparison to the more common single-point, like pressure probes or hot wire anemometry, measurements this whole field technique delivers a high spatial resolution in spite of a limited temporal resolution.

\subsubsection{Measurement plane}

The measurement plane is oriented parallel to the chord and perpendicular to the dihedral line (Figure 4.3). Due to the rotation of the wing with respect to the flat laser sheet, the spanwise position of the measurement plane deviates up to $38 \%$ of the total span at maximal flap angle.

To reveal some insight in the complete three dimensional flow field around the wings, separate measurements at four different span wise locations are performed: $0.25,0.50$, 


\section{Experimental Set-up}

0.75 and $1.00 \mathrm{R}$. Instead of moving the light sheet with respect to the wings, the wings are moved with respect the measurement volume. In this manner the necessity for the recalibration of the cameras is avoided and time will be saved during the measurement runs.

The primary aim is to visualise the fluid motion in the immediate vicinity of the wing. Care must be taken that the complete set of wings and the expected vortices will appear in the measurement volume at any spanwise location. In correspondence to the theoretical analysis, the large vortex at the end of the outward motion appears on the inner side of the wings and the vortex generated at the beginning of the inward motion is expected to be small. The width of the measurement volume should be only slightly larger then the maximal wing displacement. At the largest amplitude of the leading edges, the wing tips are a distance $r$ apart corresponding to $r=2 b \sin \varphi=20.8 \mathrm{~cm}$ for wing span $b=14 \mathrm{~cm}$ and maximal flap angle $\varphi=48^{\circ}$ (Figure 4.3). The displacement of the foil never exceeds the maximal leading edge displacement.

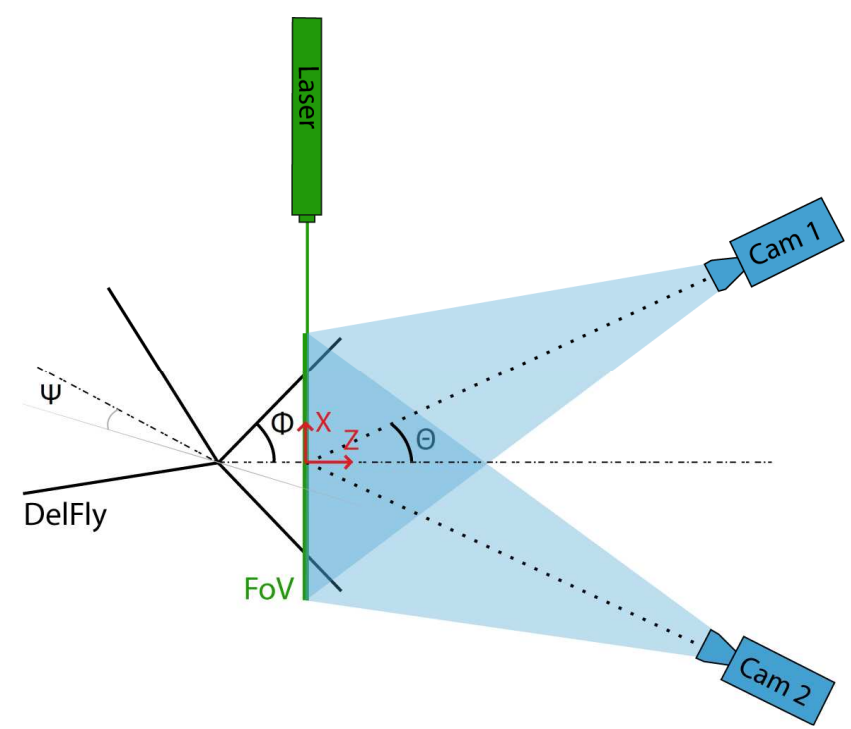

Figure 4.3 Schematic representation of the PIV apparatus.

Visualisation of the wake would reveal interesting information about the vortex interaction, omitting the high reflection involved with imaging of the wings. The maximal chord length is $8.1 \mathrm{~cm}$. Assuming that the vortices travel backwards into the wake at the induced velocity, the wake of one wing beat would extend over a distance $\mathrm{V}_{\text {ind }} / f=12 \mathrm{~cm}$. (Appendix A)

Taking these considerations and the optical access into account, a field of view of approximately $20 \times 20 \mathrm{~cm}^{2}$ is preferred (Figure 4.5). To obtain a higher spatial resolution in the region closely surrounding the wings, the same geometrical set-up is used with a lens of larger focal length, generating a field of view of $11 \times 11 \mathrm{~cm}^{2}$. 


\subsubsection{PIV apparatus}

A water-based fog of droplets with $1 \mu \mathrm{m}$ mean diameter is immersed in the flow by a Safex fog generator. The test section is filled with seeding until the concentration was sufficient, which was checked by online PIV particle recordings.

The image magnification $\mathrm{M}$ is defined as the ratio of the image to the object size. The images were recorded by two Lavision Highspeedstar CMOS cameras with 1024x1024 pixels of size $20 \mu \mathrm{m}$. The product of the pixel size and the number of pixels gives a sensor size of $2 \times 2 \mathrm{~cm}^{2}$. To obtain a field of view of $20 \times 20 \mathrm{~cm}^{2}$ a magnification of 0,1 is necessary. To accomplish this, the cameras were equipped with $35 \mathrm{~mm}$ lenses. The digital resolution attained is 5 pixels $/ \mathrm{mm}$.

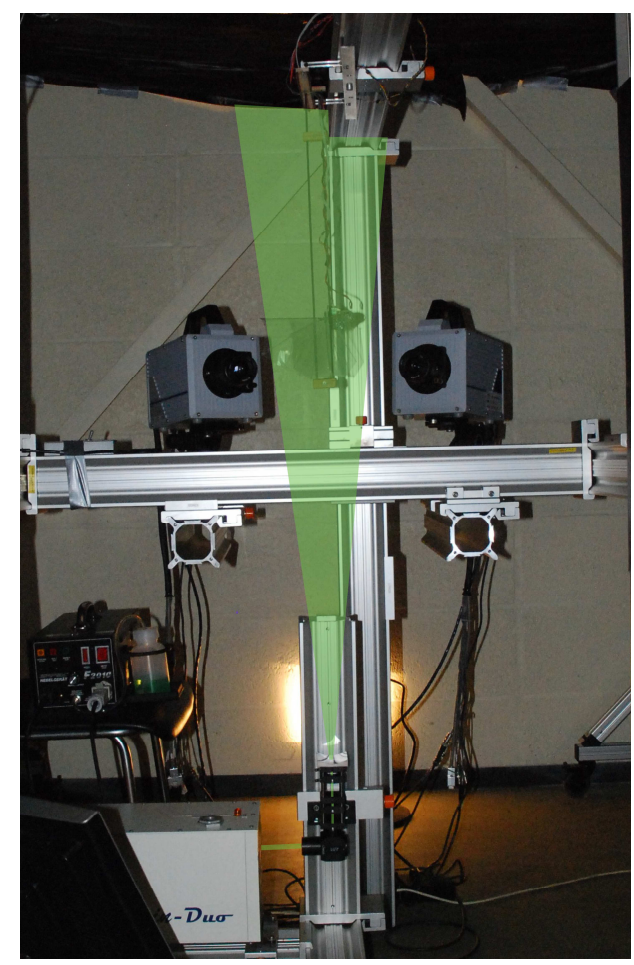

Figure 4.4 PIV apparatus

With the f-number, defined as the ratio of focal length $\mathrm{f}$ and the aperture diameter $\mathrm{D}$, set to 2.8 the imaged particles have the size of a pixel. In this manner the light scattered by the particles can be discerned from the light reflected by the wing surfaces. As a drawback, the measurements include a risk for peak locking, which is the systematic tendency of PIV evaluation of small particles to bias towards integral pixel values. Since the data are mainly used for qualitative flow description, this is not a mayor concern.

The tracer particles were illuminated by a Quantronix Darwin Duo dual oscillator, single head Nd:YLF laser, with $200 \mathrm{~ns}$ pulse duration at $527 \mathrm{~nm}$ wavelength. Measurements were taken at $200 \mu$ s pulse separation time. The maximum duration of each illumination pulse should be such that the particle displacement within the pulse duration is much smaller than the size of the particle image itself to avoid streaking. Accounting for 


\section{Experimental Set-up}

maximal in-plane velocities of $5 \mathrm{~m} / \mathrm{s}$, particle image of $4 \mu \mathrm{m}$ and pulse duration time of $200 \mathrm{~ns}$ this requirement is satisfied.

\begin{tabular}{lcc}
\hline \hline field-of-view & $202(\Delta \mathrm{x}) \times 202(\Delta \mathrm{y}) \mathrm{mm}^{2}$ & $112(\Delta \mathrm{x}) \mathrm{x} 112(\Delta \mathrm{y}) \mathrm{mm}^{2}$ \\
effective particle diameter & $1 \mu \mathrm{m}$ & $1 \mu \mathrm{m}$ \\
pulse duration & $200 \mathrm{~ns}$ & $200 \mathrm{~ns}$ \\
wavelength & $527 \mathrm{~nm}$ & $527 \mathrm{~nm}$ \\
pulse separation time & $200 \mu \mathrm{s}$ & $200 \mu \mathrm{s}$ \\
magnification & 0.09 & 0.17 \\
recording lens & $35 \mathrm{~mm}$ & $60 \mathrm{~mm}$ \\
digital imaging resolution & $5 \mathrm{pix} / \mathrm{mm}$ & $9 \mathrm{pix} / \mathrm{mm}$ \\
$\mathrm{f}_{\#}$-number & 2.8 & 2.8 \\
recording rate & $1 \mathrm{kHz}$ & $1 \mathrm{kHz}$ \\
interrogation window size & $16 \times 16 \mathrm{pixels}$ & $16 \mathrm{x} 16 \mathrm{pixels}$ \\
\hline \hline
\end{tabular}

\section{Table 4.1 Stereoscopic PIV parameters}

The mean flow is expected to be perpendicular to the stroke plane and the smallest velocity variations are expected in spanwise direction. The laser sheet is aligned with the direction of largest displacements to minimize particle pair loss. The measurement plane was oriented parallel to the chord and perpendicular to the dihedral line (Figure 4.3). The light sheet thickness was $2 \mathrm{~mm}$.

\subsubsection{Stereoscopic PIV}

The three-dimensional effects are known to be important in flapping wing aerodynamics and measurement of all three velocity components to avoid the error induced by the perspective distortion, is therefore desirable.

The cameras are placed in an angular configuration. Both cameras were placed symmetrically at an angle $\theta$ of $27^{\circ}$ with respect to the dihedral line (Figure 4.3). Corresponding to Lawson 25 this configuration would give a measurement error for the out-of-plane component of about twice that for the in-plane components. This is a generally accepted situation. The lenses were mounted on Scheimpflug adaptors to image the complete field of view in focus.

\subsubsection{Measurement series}

To be able to deduce the mechanism behind the vortex generation, the development of the flow field during the complete flapping cycle should be analyzed. The expectations with respect to the repeatability are rather low due to short model life-time, small fabrication differences between different models and deterioration of individual models and flapping cycle variability in general. Therefore time resolved PIV-measurements are preferred. At 
a recording rate of $1 \mathrm{kHz}$ series of 500 image pairs are recorded. Separate measurements at 4 different span wise locations are performed: $0.25,0.50,0.75$ and $1.00 \mathrm{R}$. The mean wing beat frequency was selected as $13 \mathrm{~Hz}$, which resembles a typical flapping frequency necessary to sustain hovering flight.

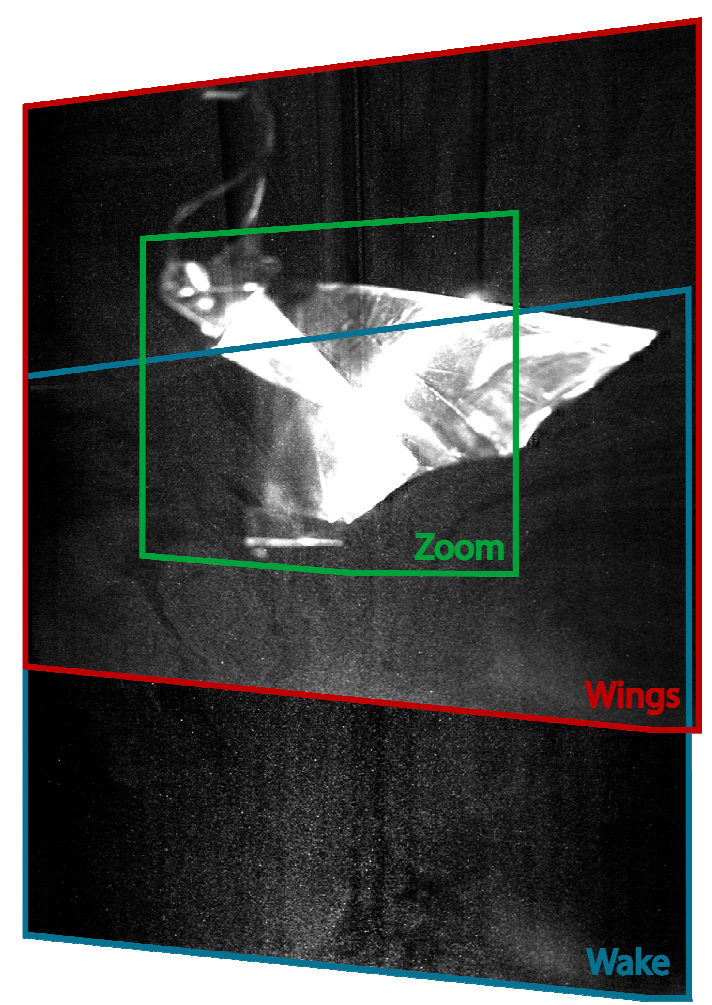

Figure 4.5 Different fields of view

\begin{tabular}{lcccc}
\hline \hline & Field of view & Spanwise position & $\begin{array}{c}\text { Flap } \\
\text { frequency }\end{array}$ & $\begin{array}{c}\text { Recording } \\
\text { mode }\end{array}$ \\
\hline Wing & $20 \times 20 \mathrm{~cm}^{2}$ & $0,25 / 0,50 / 0,75 / 1 \mathrm{R}$ & $13 \mathrm{~Hz}$ & time resolved \\
Wing & $20 \times 20 \mathrm{~cm}^{2}$ & $0,75 \mathrm{R}$ & $11 \mathrm{~Hz}$ & phase locked \\
zoom & $11 \times 11 \mathrm{~cm}^{2}$ & $0,25 / 0,50 / 0,75 / 1 \mathrm{R}$ & $13 \mathrm{~Hz}$ & time resolved \\
Wake & $20 \times 20 \mathrm{~cm}^{2}$ & $0,25 / 0,50 / 0,75 / 1 \mathrm{R}$ & $13 \mathrm{~Hz}$ & time resolved \\
\hline
\end{tabular}

Table 4.2 Test matrix

A quick analysis of the first result revealed that the background reflections largely decreased the quality of the result. With the aim to decrease the background reflections by data pre-processing and sum of correlations (see section 4.2.6), a subsequent series of phased locked measurements were performed. A series of pictures are recorded with the wings in approximately the same position. Unfortunately, by this time the model's lifetime already largely exceeded the average DelFly-lifetime and different parts of the driving system suffered from deterioration. Because of this it was necessary to decrease the flapping frequency to $11 \mathrm{~Hz}$, which is not a realistic value for hover, and restrict measurements to one spanwise location. Series of 150 image pairs are recorded at the 


\section{Experimental Set-up}

spanwise position $0.75 \mathrm{R}$ at 6 different moments in the flap cycle: $0.2,0.32,0.44,0.65$, 0.80 and $0.94 \tau$. The non-dimensional time $\tau$ is defined as the ratio of time instant and cycle period. The moment when the wings are at minimal stroke angle is chosen as $\tau=0$.

\subsubsection{Image analysis}

The effect of background reflections on the images of the time resolved measurement series was reduced by min/max normalization (Figure 4.6), which implies that each pixel intensity is normalized using the local minimum and maximum intensities determined over sub-regions. The phased locked measurement series provide the possibility to reduce the background reflections by subtracting the minimum intensity calculated over a range of images whereon the wings are in approximately the same position (Figure 4.7).
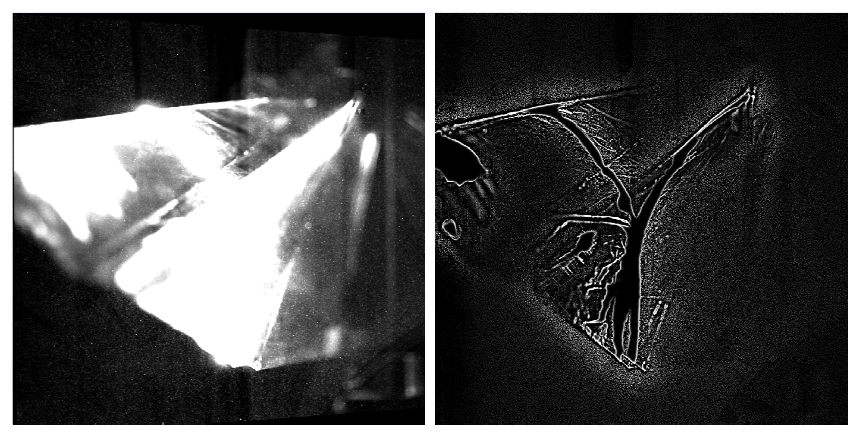

\section{Figure 4.6 Reduction of background reflection for time resolved measurements} left: raw image; right: $\min / \max$ normalization

Velocity vectors are calculated by FFT-based cross-correlation. The size of the interrogation window should be sufficient large, such that the majority of particles are imaged in the same interrogation window at both exposures to be able to estimate the correct displacement vector. However, the spatial resolution decreases with increasing window size. To achieve the high signal-to-noise ratio from a large window size and the high spatial resolution from the small interrogation window, a multi-pass interrogation with window-offset is applied. Hereby the correlation starts on a grid with interrogation window size of 32 by 32 pixels. After the first pass, the interrogation window size is decreased to 16 by 16 pixels and the interrogation widow of the second exposure is offset by the displacement calculated in the first pass. The spatial resolution is further increased ultimately using an overlap of 50\%. A field of $128 \times 128$ vectors with a vector grid spacing of $1.5 \mathrm{~mm}$ is acquired.

Spurious vectors are sorted out using a Median test ${ }^{26}$ and replaced using interpolation. The intensity maps are used to mask the region where the data are unreliable because of the high reflectivity of the wing foil.

Calibration, data acquisition and data processing are carried out using Davis 7.3 software. 

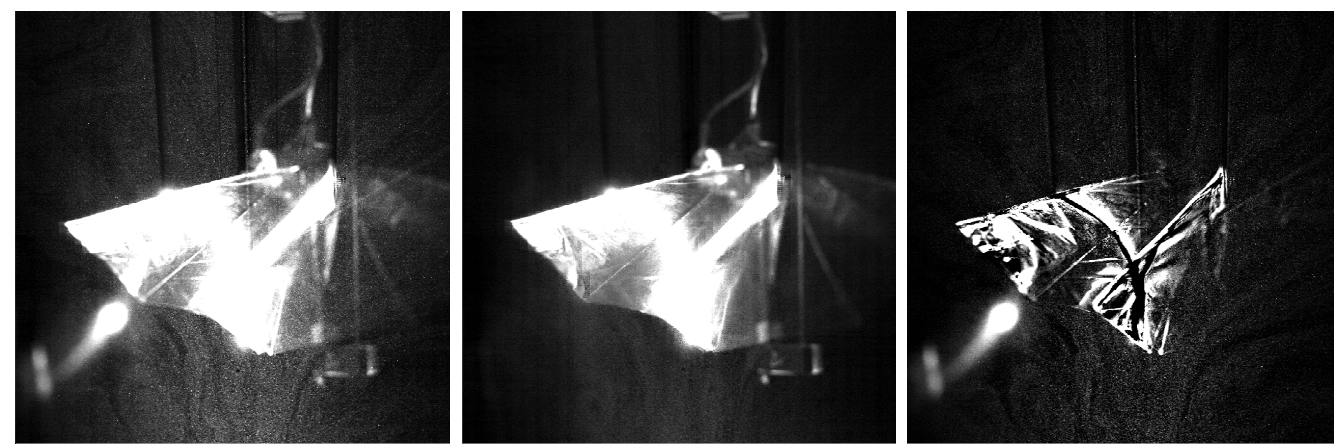

Figure 4.7 Reduction of background reflection for phase locked measurements left: raw image; middle; minimal intensity; right: minimal intensity subtracted from raw image

\subsubsection{Vortex identification}

To understand the fluid dynamics involved, visualization of the vortex structure would be beneficial.

The most straightforward would be to use the magnitude of the out-of-plane vorticity vector $\xi_{z}=\partial u / \partial y-\partial v / \partial y$. However, the magnitude of the vorticity is unable to identify vortex cores embedded in a shear flow. The regions around the wings we are interested in are large shear regions. In frame b of Figure 4.8 we see that the vortex cores in the wake can clearly be visualized by a contour plot of the magnitude of the vorticity. But vortices in the region between the wings are overshadowed by the high shear.

To distinguish the shear and rotation, the Q-method ${ }^{27}$ can be used. The second invariant of the fluid gradient tensor is defined as

$Q=1 / 2\left(\Omega_{i j} \Omega_{i j}-S_{i j} S_{i j}\right)$

where $S_{\mathrm{ij}}$ and $\Omega_{\mathrm{ij}}$ respectively are the symmetric and anti-symmetric component of the velocity gradient tensor:

$S_{i j}=1 / 2\left(\nabla u+(\nabla u)^{t}\right)$ and $\Omega_{i j}=1 / 2\left(\nabla u-(\nabla u)^{t}\right)$.

As such positive values of $\mathrm{Q}$ can be interpreted as regions where the rotation is dominant over the shear. In frame $\mathrm{c}$ of Figure 4.8 the values of $\mathrm{Q}$ are shown. We see that this method successfully leaves the shear regions out in determining the vortex cores. However, this condition does not imply that a pressure minimum occurs within the region defined as a vortex core.

Alternatively, the $\lambda$-method ${ }^{28}$ determines the existence of a local pressure minimum due to vortical motion. The occurrence of a local pressure minimum requires two positive eigenvalues of the Hessian of the pressure. Neglecting unsteady straining and viscous effects in the anti-symmetric part of the gradient of the Navier-Stokes equation, the Hessian of the pressure is defined as 
$-p_{i j}=\rho\left(S_{i k} S_{k j}+\Omega_{i k} \Omega_{k j}\right)$.

Consequently a vortex core is defined as a region with two negative eigenvalues of $\left(S_{i k} S_{k j}+\Omega_{i k} \Omega_{k j}\right)$.

In frame $\mathrm{d}$ of Figure 4.8, the value of the second eigenvalue is shown. The regions identified as vortex cores largely correspond to the result obtained with the Q-method.
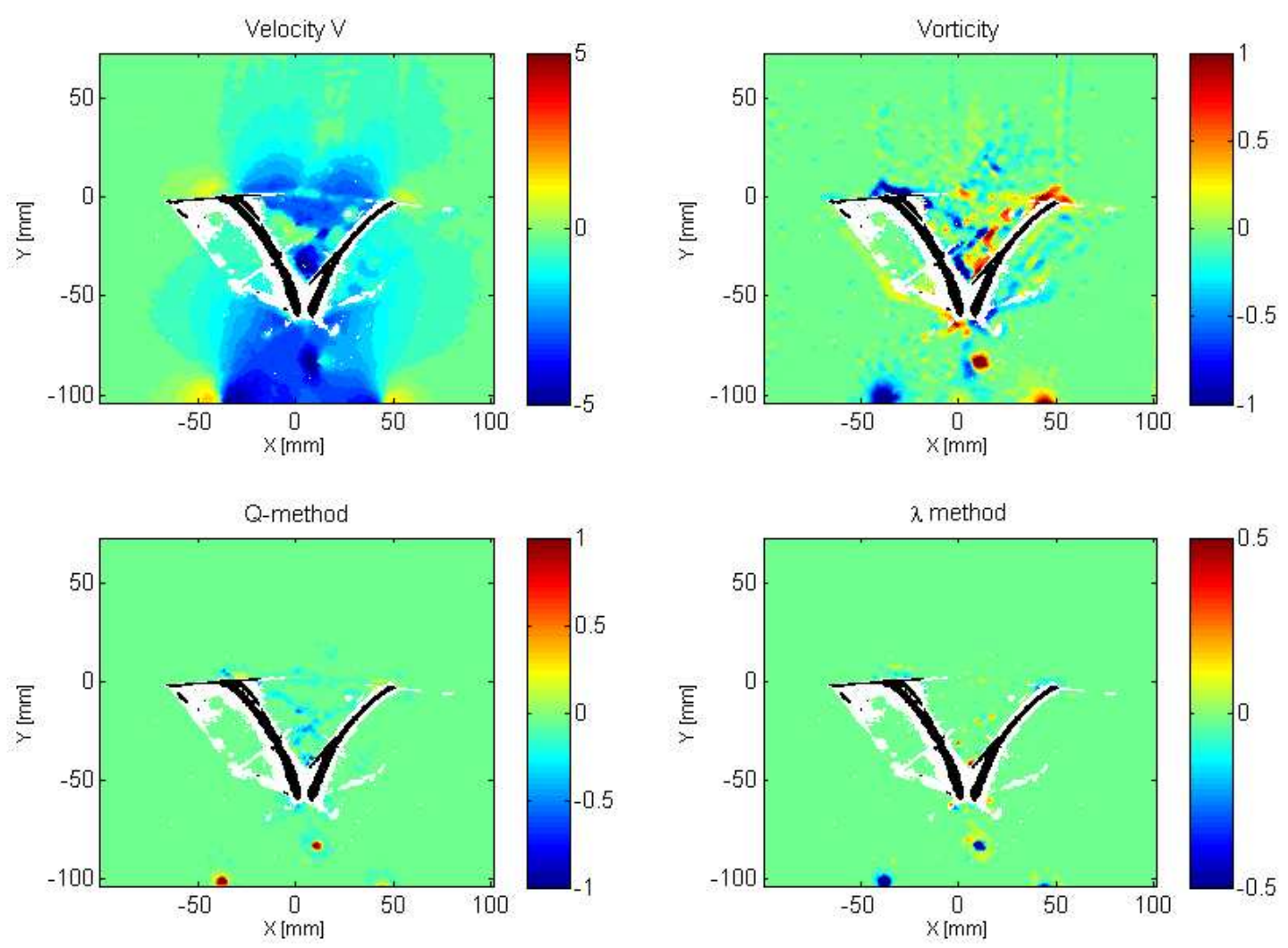

Figure 4.8. Visualization of vortex topology using different techniques.

\subsection{Force measurement}

Using two carbon tubes reinforced with wooden clamps to minimize lateral vibrations, the wings hang rigidly on a strain-gauge balance with two Wheatstone bridges. The vertical forces were measured and recorded at a sampling rate of $1000 \mathrm{~Hz}$. Miniature sensors Q70x5x9-H with a capacity of $20 \mathrm{~g}\left(\mathrm{~g}=9.81 \mathrm{~m} / \mathrm{s}^{2}\right)$ were used. Using a Picas V2.6.1 Multi-channel amplifier system the range was set from $+40 \mathrm{~g}$ to $-40 \mathrm{~g}$. 


\subsection{Force measurement}
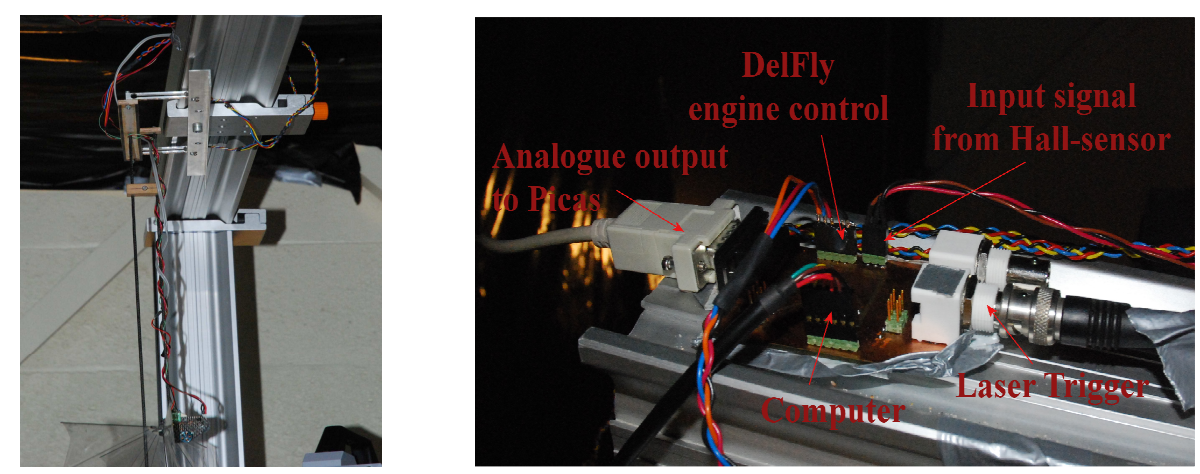

Figure 4.9 a) the strain-gauge balance and b) measurements synchronization system

The PIV system and the flapping-wing device are synchronized and measurements are triggered at specific wing positions. The triggering is done by the $\mathrm{PC}$ controlling the wing motion using C++-based software. A Hall-sensor attached to the gear box recorded the starting point of a cycle. By tracking the motor pulses, the wing location and the force measurement can be correlated. Synchronization between the laser and the cameras is achieved by the Lavision Controller operated through Davis 7.3 software. 
4 Experimental Set-up 


\section{Results}

Using the test apparatus described in chapter 4, the flow field around a hovering DelFly has been studied.

In section 5.1 we first visualize the trajectory of the imaged wing section, in order to investigate whether its trajectory resembles that of our theoretical discussion in chapter 3 . Linking the measured forces to the wing attitude, the flap phases attributing to the lift enhancement are distinguished in section 5.2. The aerodynamic mechanisms underlying the lift enhancement and attenuation are investigated in an analysis of the PIV results in section 5.3. The development of typical lift enhancing mechanism is discussed in more detail for both the clap and fling mechanism and the flex mechanism along with the effect on the subsequent stroke in section 5.3.1 and 5.3.2 respectively.

The time resolved and phased locked measurements were performed at different flapping frequencies. Comparing measurements of the two measurement series, the influence of flapping frequency on force generation and fluid structure will be specified in section 5.3.3.

\subsection{Observation of the wing motion and deformation}

With the high speed camera operating at $1 \mathrm{kHz}$ a time resolved series of images was recorded of the wings flapping at a frequency of $13 \mathrm{~Hz}$. Observation of a wing section at $3 / 4$ span is discussed in this section. By analyzing the raw pictures, the position and the shape of the wing section in the measurement plane could be traced. The wing deformation due to the high flexibility and the heaving motion of the leading edges is revealed. In Figure 5.1, different stages of the cycle are represented by the nondimensional time $\tau$, the time instant divided by the cycle period. The in- and outstroke are shown in the upper and lower part of the figure respectively.

Starting from the instant that the leading edge spars are closest together and the wing surface touch, the leading edge spars move apart at increasing velocity. The point of contact moves along the wing towards the trailing edges. The wing foil peels apart under high deformation. At the end of this phase, called the fling (label A), the trailing edges separate and the wings continue moving outward at a more constant speed and angle of attack (label B). At the maximum wing stroke amplitude the leading edge spars decelerate and reverse direction (label $\mathrm{C}$ ). The trailing edges temporally stay stationary and the wing flexes (label D). The leading edge accelerates in the opposite direction and the foil is tightened. Under this condition the wing sections move inwards (label E). 


\section{Results}

Approaching each other, the leading edge spars decelerate causing the camber to decrease and the wing foils to clap together (label F). Instead of rigidly clapping the wings together, the clap is more akin to a reverse peel. Already before the clap has been completed the leading edges start separating again, pulling the wing surfaces apart.

During the acceleration in the beginning of the translational stroke, the wing foil pulls tight and the leading edge is pulled downwards (label A and D). At the end of the stroke, tension is released and the leading edge moves back towards its original position (label $\mathrm{C}$ and F). The wing tip trajectory with respect to the body is an elongated figure eight-shape. Thanks to the upward motion during clap, the foil is pulled tight during the rotation. The downward leading edge motion during peel might reinforce the leading edge vortex generation by increasing the velocity of the fluid moving into the opening gap ${ }^{29}$.
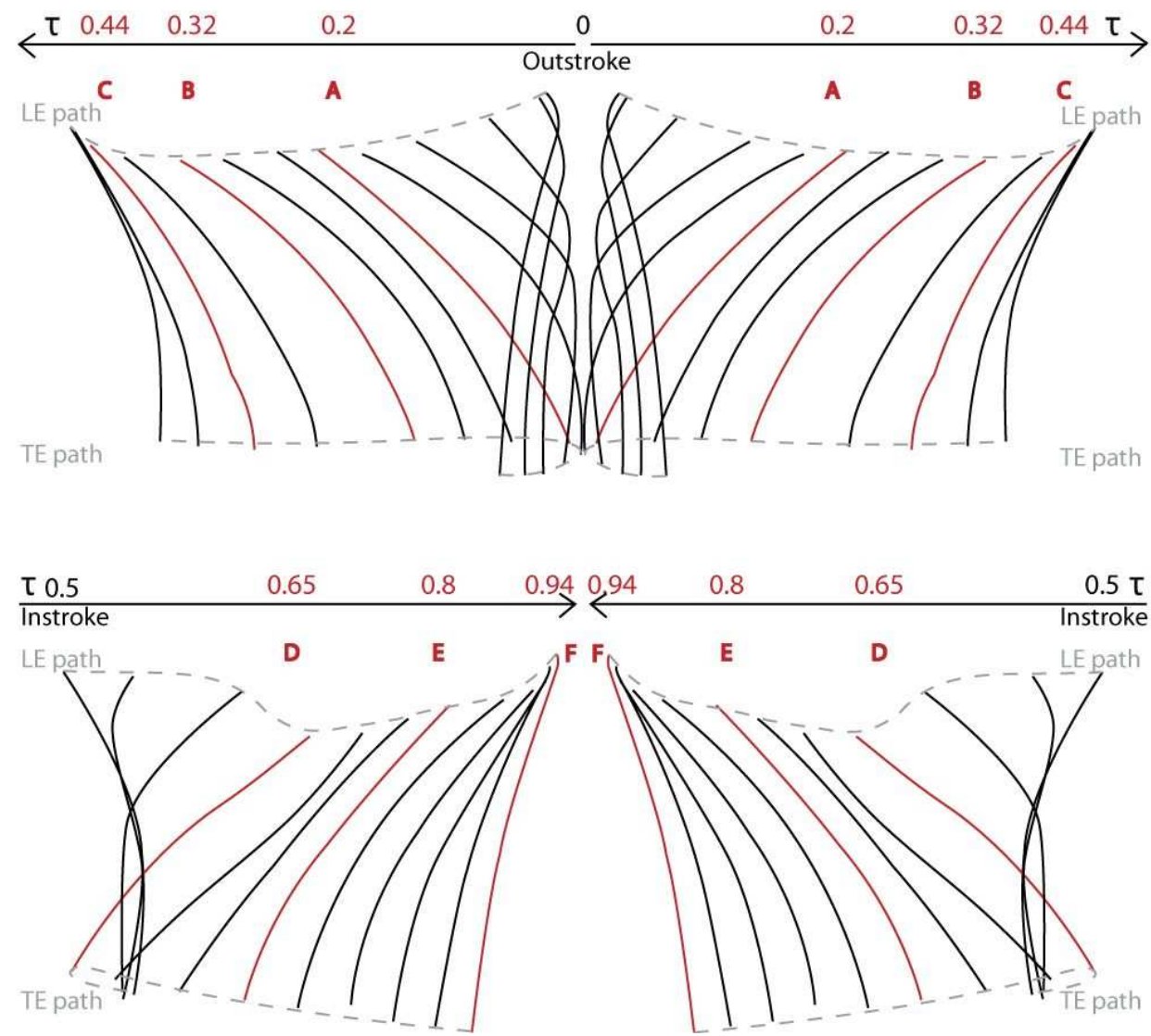

Figure 5.1 Schematic representation of the wing motion during one flap cycle defined from visual inspection of the PIV recordings. In- and outstroke represented by upper and lower part respectively

The images clearly reveal the strong deformation of the flexible wing foil and leading edge, which except for the early onset of the peel, indeed resembles the figures shown before. 


\subsection{Force measurements related to wing motion}

At the same moment the images were recorded, the upward force exerted by the flapping wings has been measured using a strain-gauge balance. The force fluctuations with respect to the non-dimensional time are shown in Figure 5.2, along with the labels A to F corresponding to the red wing sections in Figure 5.1. The error bars indicate the standard deviation of the force measurements over the complete measurement run. The mean force over one cycle attains $0.21 \mathrm{~N}$, which is largely sufficient to lift a DelFly II typically weighting 17 grams. The maximal force amounts to $0.69 \mathrm{~N}$ while the second peak value of $0.56 \mathrm{~N}$ is $17 \%$ lower.

During the peel-phase a strong increase in force is established, with a peak value occurring immediately after the trailing edges have separated (A). Before the isolated rotation the force drops to slightly negative values (C). At the end of the isolated rotation, after the foil has been pulled tight, a second peak force appears (D). A gradual decrease ends in a second negative peak when the leading edge spars reverse direction at the end of the instroke $(\mathrm{F})$.

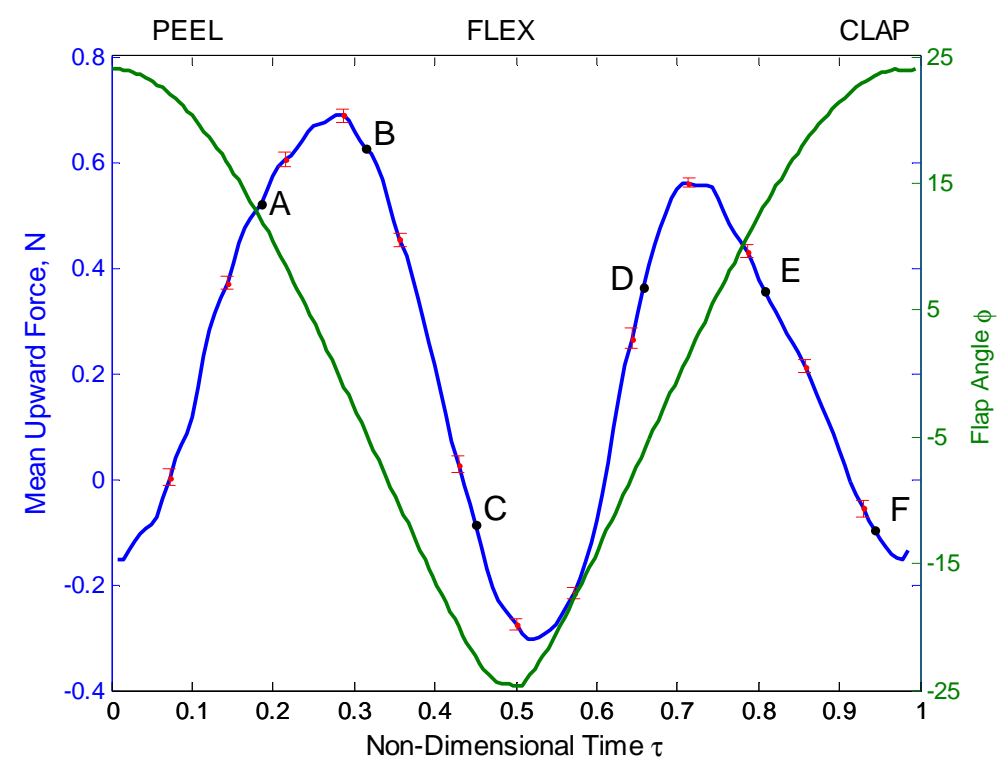

Figure 5.2 Upward force fluctuations and flapping angle during one flap cycle for $f=13 \mathrm{~Hz}$. Error bars show standard deviation. Labels A to F correspond to the redcolored wing sections in Figure 5.1

Considering this specific type of wing motion and the striking force fluctuations it is expected that the peel mechanism and the occurrence of a leading edge vortex at the isolated rotation contribute to the lift generation. The reasons for the recession in lift at the end of the outstroke and during the clap are unclear but could be explained by the tip of the leading edge flexing upwards.

We will now look into the PIV measurements and simultaneous force measurements to make a link between fluid dynamics and forces. 


\section{Results}

\subsection{Fluid dynamics related to force generation}

The experiments yielded a huge amount of information. Discussion of all this data is beyond the scope of this report. A few results are selected to demonstrate the core issue of this study. A complete overview of data is assimilated in the appendices. Appendix B contains an overview of the time resolved PIV images at all time instants discussed in this section for the different spanwise locations. In appendix $\mathrm{C}$ the corresponding vortex visualizations, using the $\lambda$-method, can be found. The vortex visualization of the phased locked PIV measurements discussed in section 5.3.3 are shown in appendix D. Appendix $\mathrm{E}$ and $\mathrm{F}$ contain the flow field of the zoom and the vortex visualization of the wake respectively.
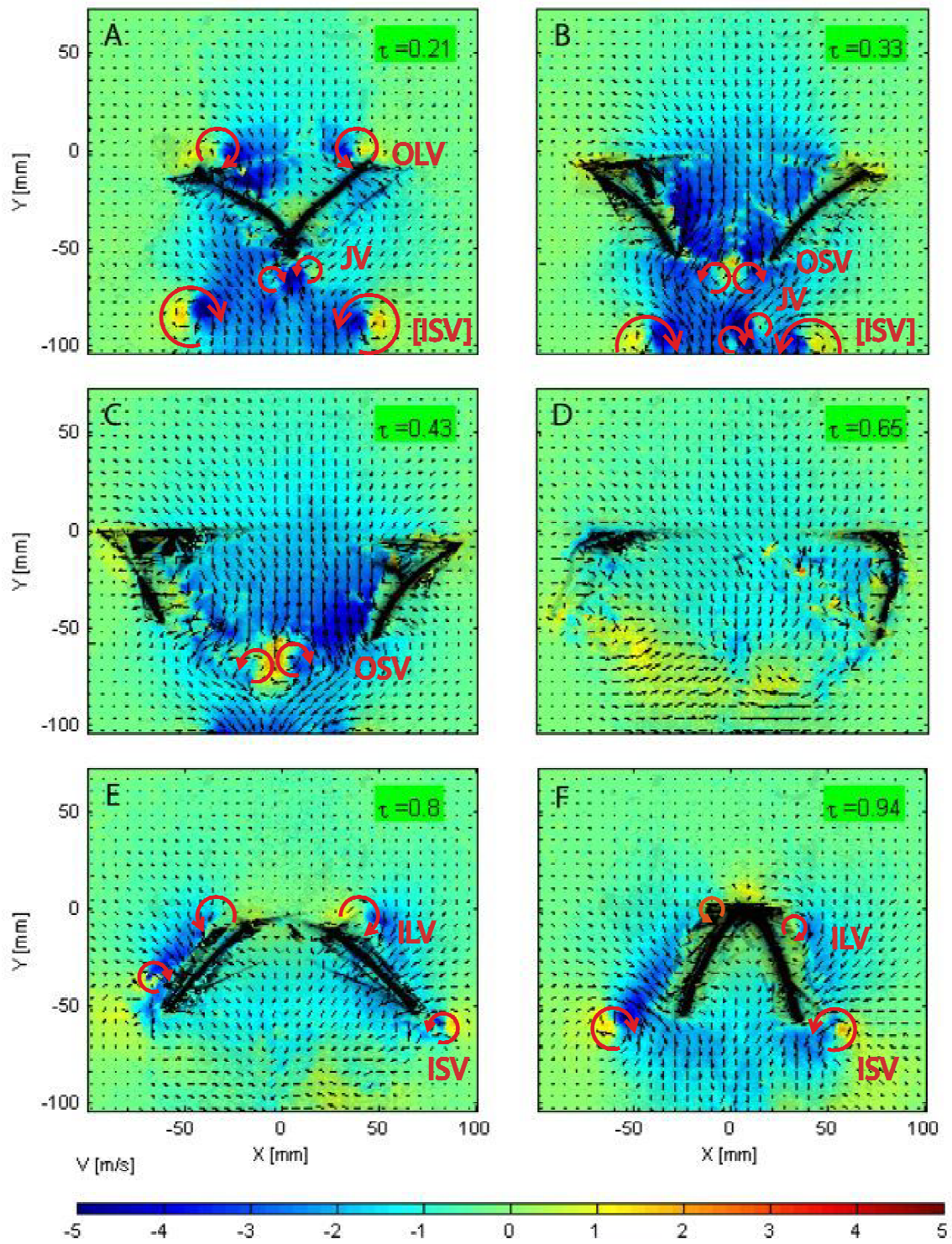

Figure 5.3 Time resolved PIV results for $b=0.75 \mathrm{R}$ and $f=13 \mathrm{~Hz}$ 
PIV measurements were performed at various instances during a flapping cycle, whereat the force measurements labeled in Figure 5.2 were performed and the wings were positioned as indicated by the red-colored sections in Figure 5.1. Using the motor pulse as a reference signal, the PIV measurement taken at a specific moment during the flap cycle could be linked to the instantaneous force measurement.

Figure 5.3 shows the in-plane velocity component of the 3D vector field of the different time resolved PIV measurements. The measurement plane is positioned at $75 \%$ span with a deviation of $38 \%$ span at maximal flap angle due to the rotation of the wing with respect to the flat laser sheet. The color-contours indicate the magnitude of the vertical velocity component. The black-colored regions represent the areas were data are compromised due to shadows, optical obstructions or background reflections.

The first measurement, represented in frame A, is taken at the moment when the trailing edges separate at the end of the peel. Even when disregarding the vector field in the area between the wings, where the data are unreliable because of the high reflectivity of the wing foil, around the leading edges a swirl of air into the gap identified as leading edge vortices can be clearly distinguished. This outstroke leading edge vortex is indicated in Figure 5.3 with OLV. Two small vortices in the same direction are distinguished under the trailing edges. These vortices are assumed to originate in the rolling up of the jet flow created by the clap motion and are indicated in the figure by JV (jet vortex). The upward force increases under these conditions.

After the lifting force has reached a peak value, it has started to decrease. In frame B, the trailing edges have separated and the wings move further outward at more constant velocity and shape. An outstroke starting vortex (OSV) is shed from both the trailing edges and a strong downwash of air between both wings is clearly visible. The occurrence of a leading edge vortex cannot be assessed unambiguously from the PIV result because of the poor accuracy near the wings, but is doubtful since the inrush of air decreases the angle of attack considerably.

When the wings move further apart the lift decreases considerably until the last moment before rotation at the maximal stroke angle, represented in frame $\mathrm{C}$. The drop in lift is supposedly attributed to the reaction force on the abrupt upward motion of the leading edges (Figure 5.2) and the starting vortex still present in between the wings counteracting the buildup of bound circulation.

Subsequently, the leading edges change direction and the wing foil flips around. In frame $\mathrm{D}$ the wing foils flex and the leading edges accelerate during the in- and downward motion. Although the force increases, no leading edge vortex is visible in the PIV image. In frame $\mathrm{E}$, the wings have approached till half of the maximal stroke angle. The PIV picture suggests the appearance of a leading edge vortex. A second peak in the upward force supports this expectance. Nonetheless this peak is $17 \%$ lower then the first one. A starting vortex sheds from the trailing edge. Whether the Instroke Leading edge Vortex (ILV) and Instroke Starting Vortex (ISV) are developed corresponding to the theory of 


\section{Results}

the flex mechanism can not be derived from these images. The upward force continues decreasing.

In the initial phase of the clap, the leading edges come together as in frame F. There is no indication of air being expelled downwards. Oppositely air is being expelled upwards from the clapping leading edges, contributing negatively to the lift force and the leading edges flex upwards inducing a negative reaction force (Figure 5.2). The starting vortex slowly moves downstream into the wake. In frame A and B, the Instroke Starting Vortex of the previous cycle is indicated with square brackets.

We will now look into more detail to the time resolved measurement series to deduce the mechanisms behind the vortex generation and discuss the lift enhancing mechanisms.

\subsubsection{Clap and peel mechanism}

We will look both at the development of the flow field in time for the clap and peel mechanism (Figure 5.4) and the variation of the flow field along the span on a moment during the clap (Figure 5.6) and peel (Figure 5.8) respectively. A visualization of the vortex identification method for the same time instants and spanwise locations can be found in appendix $\mathrm{C}$.

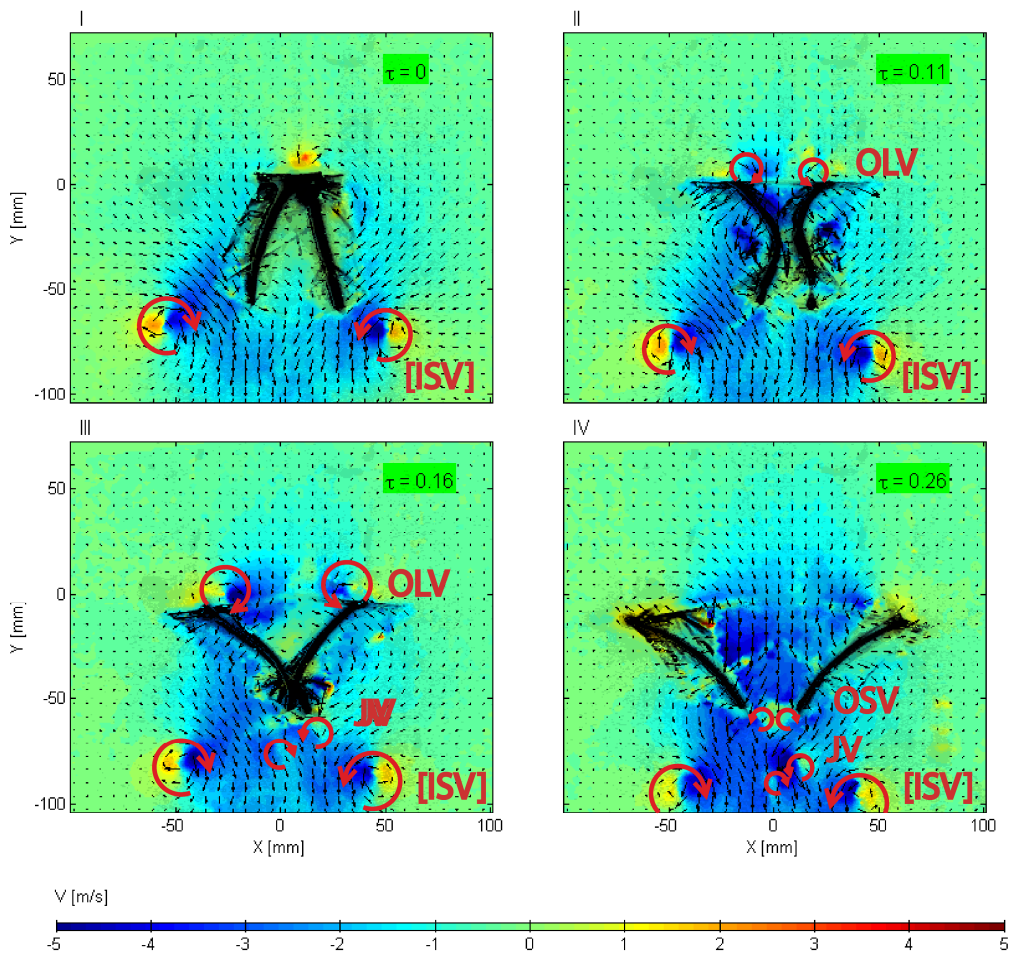

Figure 5.4. The clap and peel mechanism. $V$-component of the velocity for $b=0.75 R$ and $f=13 \mathrm{~Hz}$ 
In the initial phase of the clap, the leading edges come together and the angle of attack increases until the wings are positioned almost parallel to each other. The magnitude of the vertical velocity component under the wings is the sum of the jet velocity, the induced velocity and a contribution of both trailing edge vortices. The induced velocity is approximated as $1.5 \mathrm{~m} / \mathrm{s}$ (Appendix A). The velocity contributed by the vortex has the same size but opposite direction of the velocity in the point located symmetrically with respect to the vortex core. Subtracting the induced and vortex velocity from the measured velocity, the jet velocity is derived. As such it is concluded that near the root, where the foil is more rigidly attached, the clap does produce a downward expelling jet (Figure 5.6 I) with a velocity of $1.77 \mathrm{~m} / \mathrm{s}\left(\mathrm{V}_{\text {jet }}=\mathrm{V}_{\text {tot }}-\mathrm{V}_{\text {ind }}-\mathrm{V}_{\text {vort_left }}-\mathrm{V}_{\text {vort_right }}=4.39-1.5-0.23-0.9 \mathrm{~m} / \mathrm{s}\right)$. In contrary to Weis-Fogh's ${ }^{2}$ suggestion, no expelling downward jet could be observed during the clap at $3 / 4$ span (Figure 5.6 III). This is attributed to the high flexibility of the wing foil.

Oppositely air is being expelled upwards from the clapping leading edges. The upward expelling jet above the leading edges has similar strength along the span (Figure 5.6). The force generated by the downward jet seemingly is insufficient to overcome the negative contribution of the upward jet and the reaction force to the upward motion of the leading edges (Figure 5.2).

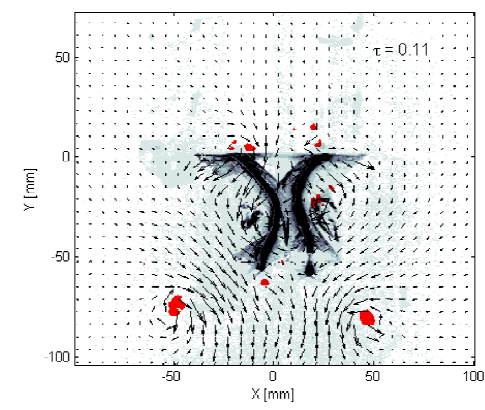

Figure 5.5 Vortex identification using $\lambda$-method at $\tau=0.11$ for $0.75 \mathrm{R}$ and $\mathrm{f}=13 \mathrm{~Hz}$ Red regions indicate negative $\lambda_{2}$ values.

Already before the clap has been completed, the leading edges start separating again, the wings curve toward the opposite side and the surfaces peel apart. The leading edge vortices (OLV), created by the strong acceleration of the flow around the leading edges, are first visible on the PIV images at $\tau=0.11$ (Figure 5.4 II, Figure 5.5) on 3/4 spanwise position. The OLV, developed due to the peel, originates later and increases in size and strength from the wing root to the tip (Figure 5.8). The higher leading edge acceleration near the wing tips supposedly is favorable for the generation of a OLV. The OLV grows in time (Figure 5.4 II-III), which concurs with Ellington's ${ }^{30}$ analysis of the peel mechanism. Accordingly the lift force increases steadily. 


\section{Results}
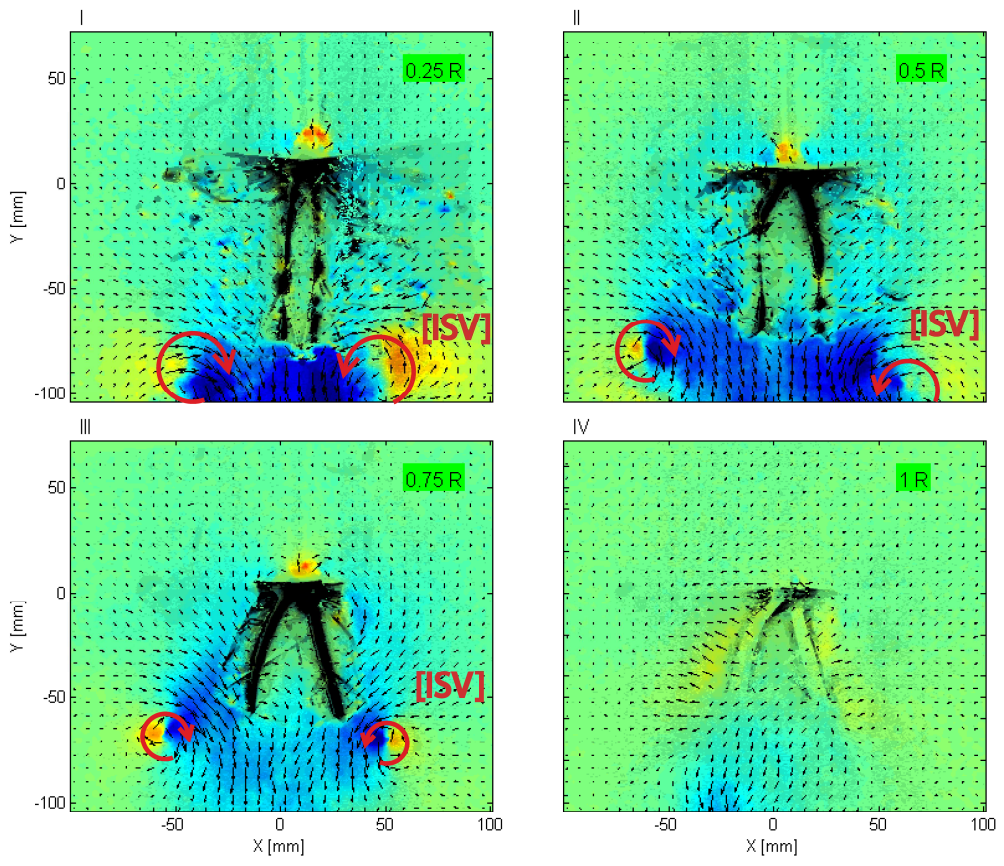

$\mathrm{V}[\mathrm{m} / \mathrm{s}]$

Figure 5.6 Spanwise variation in V-component of velocity during the clap for $f=13 \mathrm{~Hz}$ and $\tau=0$

When the contact point reaches the trailing edges the end of the clap phase is reached. Opposite to what would be expected from Weis-Fogh's description of the wing-wing interaction, a vortex is visible near both trailing edges (Figure 5.4 III, Figure 5.7). These vortices are in the opposite direction of the expected stopping vortex. Most probably the air expelled by the clap rolls up into a vortex (JV). Whether the stopping vortices are eliminated by the presence of the mirror wing or counteracted by the jet vortices rests unknown. Since the jet vortices are in the same direction as the bound circulation of the subsequent stroke, they do not hinder the build up of circulation. The jet vortices are found in the downstream induced by the clap motion and consequently travel relatively fast into the wake.

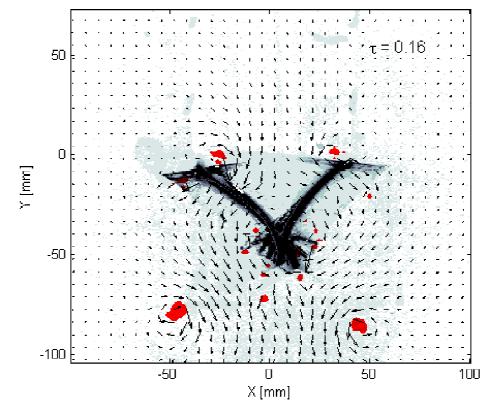

Figure 5.7 Vortex identification using $\lambda$-method at $\tau=0.16$ for $0.75 \mathrm{R}$ and $\mathrm{f}=13 \mathrm{~Hz}$ Red regions indicate negative $\lambda_{2}$ values 
An influx of flow from above fills the complete opening formed between the separating wings (Figure $5.4 \mathrm{IV}$ ) while the outstroke leading edge vortex decreases in size and strength. The inrush of air into the opening gap is stronger and lasts longer near the wing root compared to the situation at $3 / 4$ wingspan.
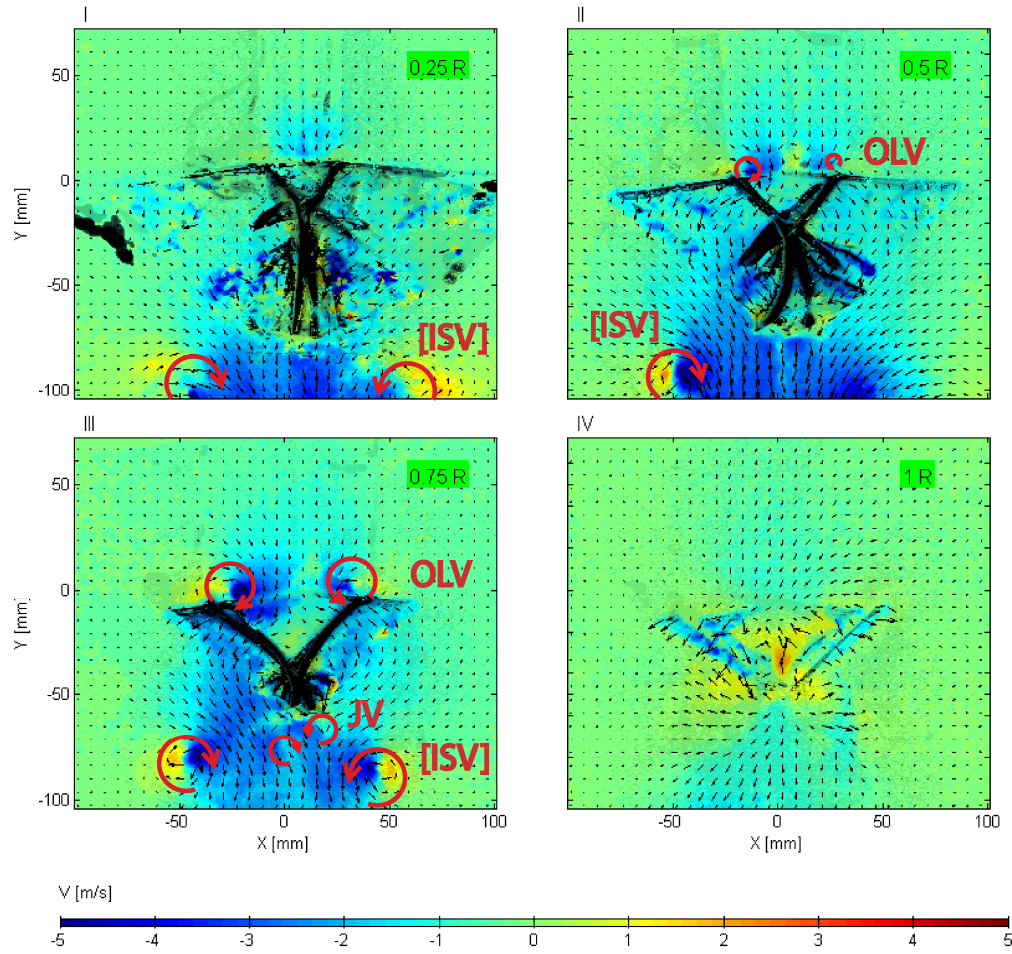

Figure 5.8 Spanwise variation in $\mathrm{V}$-compoment of velocity during the peel for $f=13 \mathrm{~Hz}$ and $\tau=0.16$

After the trailing edges have separated, a starting vortex (OSV) is shed from both trailing edges (Figure 5.9). The trailing edges separate along the complete span with some time delay starting from the tip. The start vortices shed from the separating trailing edges at the end of the peel, increases in size from root to tip. Nonetheless the high peak value in lift force attained at the end of the peel phase indicates that the peel mechanism is very effective in lift generation.

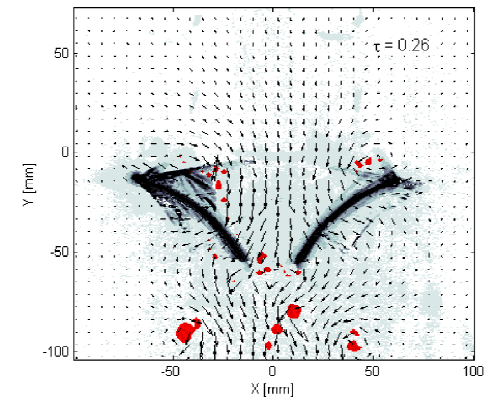

Figure 5.9 Vortex identification using $\lambda$-method at $\tau=0.26$ for $0.75 \mathrm{R}$ and $\mathrm{f}=13 \mathrm{~Hz}$ Red regions indicate negative $\lambda_{2}$ values 


\section{Results}

In the subsequent translational stroke the wings continue moving outward at a more constant velocity and angle of attack. The leading edge vortex has disappeared and the strength of the downwash diminishes. Accordingly the lift force decreases to a minimal value. The start vortex is left behind in between the wings and travels slowly into the wake.

\subsubsection{Flex mechanism}

We will look both at the development of the flow field in time for the flex mechanism (Figure 5.10) and the variation of the flow field along the span on a moment during the flex (Figure 5.11) and at the beginning of the instroke (Figure 5.12) respectively. A visualization of the vortex identification method for the same time instants and spanwise locations can be found in appendix $\mathrm{C}$.

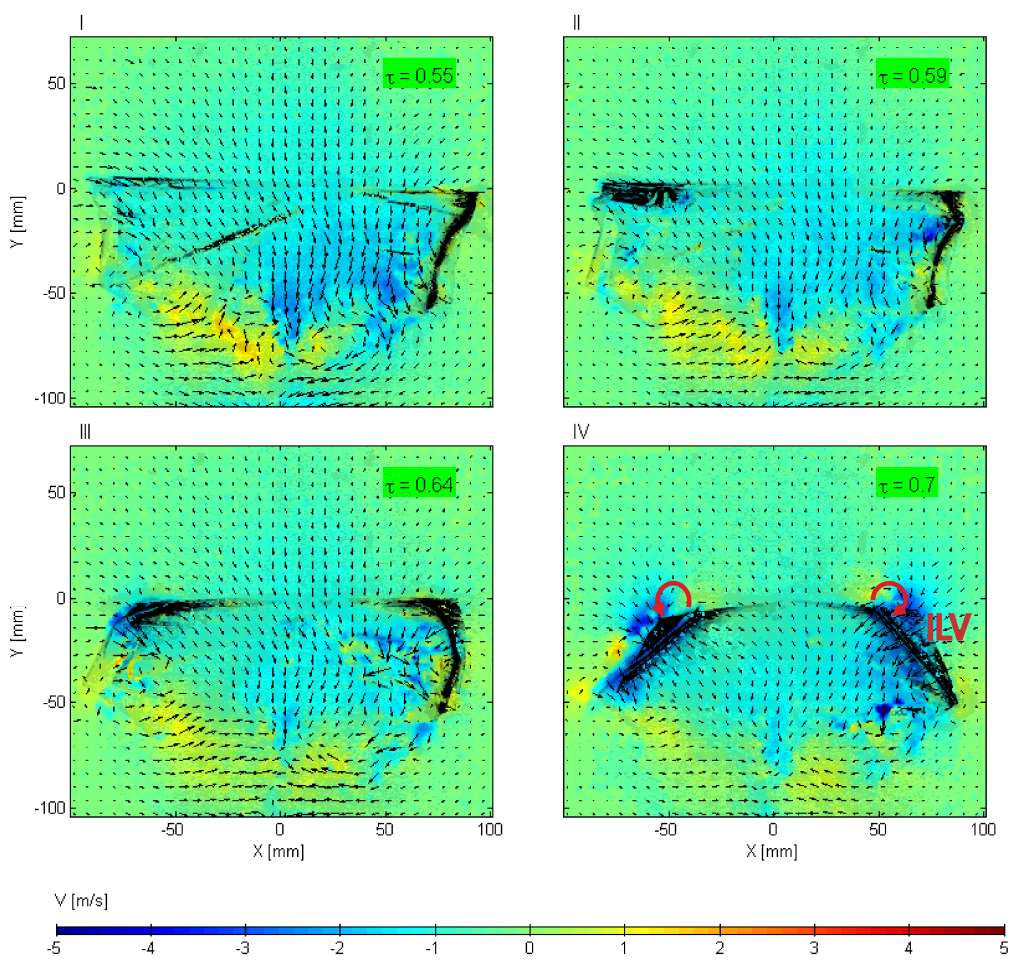

Figure 5.10 The flex mechanism.

$V$-component of the velocity for $b=0.75 R$ and $f=13 \mathrm{~Hz}$

At the end of the outstroke the leading edges slow down and the angles of attack increase until the wings are positioned almost vertically. Subsequently the wing flexes: the upper part of the wings rotate, while the trailing edges stay almost stationary. Opposite to the theory of the flex mechanism, the formation of a leading edge vortex can not be assessed from the PIV results (Figure 5.10 I-III) at none of the spanwise locations (Figure 5.11). The PIV measurement in the region where the vortex is supposed to appear is obstructed 
by the shadow from the leading edge bar and the reflections on the wing foil. Moreover the lift force steadily increases. Therefore the leading edge vortex is suspected to exist.

Since no stopping vortex is shed, in conformity with the theoretical discussion, continued circulation enhanced with rotation is thought to contribute to the lift force. Also the downwash of air between the inner half of the wings (Figure 5.11 I-II), persistently contributes to the lift force in addition to the reaction force on the downward motion of the leading edges. A peak value is attained at $\tau=0.73$. Nonetheless this peak value is $17 \%$ lower then the maximal value reached at the end of the peel.

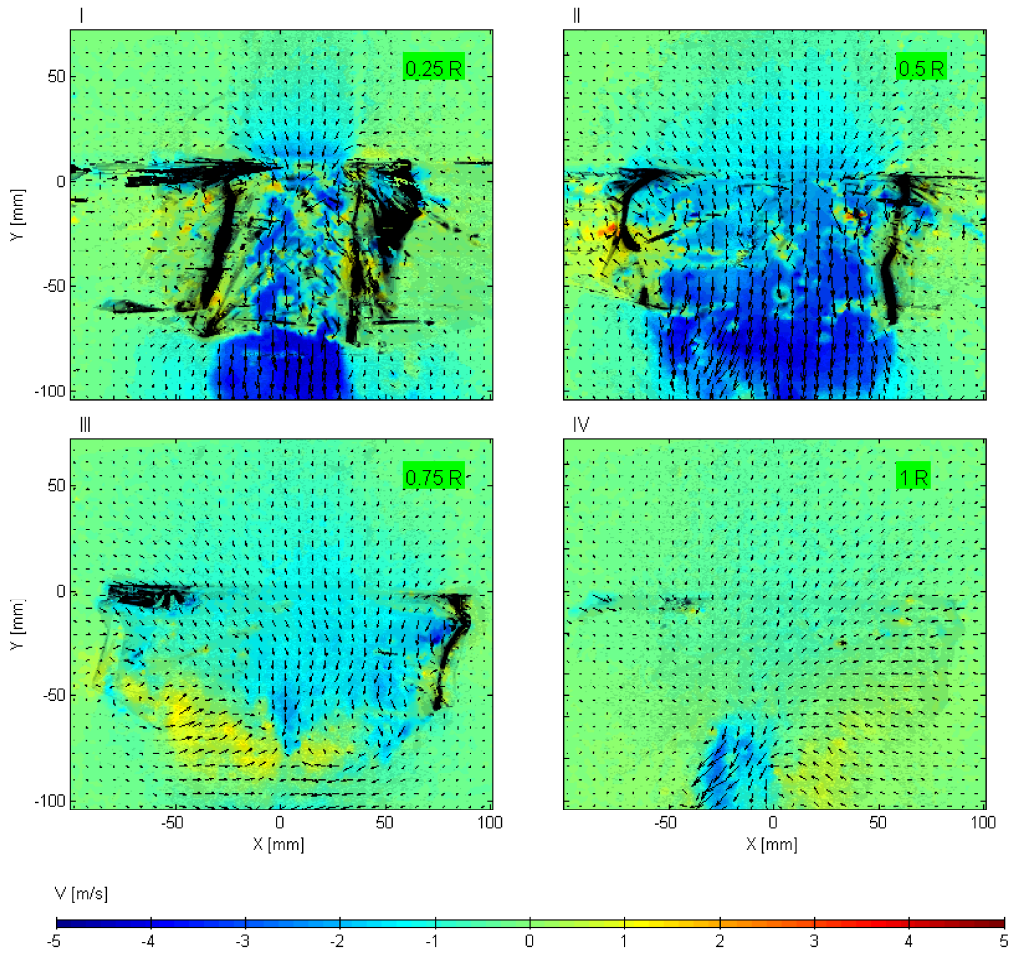

Figure 5.11 Spanwise variation in V-component of velocity during the flex for $f=13 \mathrm{~Hz}$ and $\tau=0.59$

At $\tau=0.73$ the leading edges accelerate towards the neutral position and the wing foils unflex. The wings abruptly accelerate and the angle of attack is highest. These conditions correspond to the conditions necessary for the generation of a separation bubble as described by Ellington ${ }^{3}$. The instroke leading edge vortex (ILV) first appears on this moment (Figure 5.12 III) at 3/4 spanwise position. A combined start and stop vortex, shed at the trailing edge (ISV), decreases in size and strength from root to tip (Figure 5.12).

The wings continue moving to the neutral position at a more constant speed and angle of attack during the subsequent translational phase. When disregarding the vector field in the area around the wings, where the data are unreliable because of the high reflectivity of the wing foil, a leading edge vortex (ILV) can be distinguished in frame II and III of Figure 5.12., which seems to increases in size from root to tip. 


\section{Results}

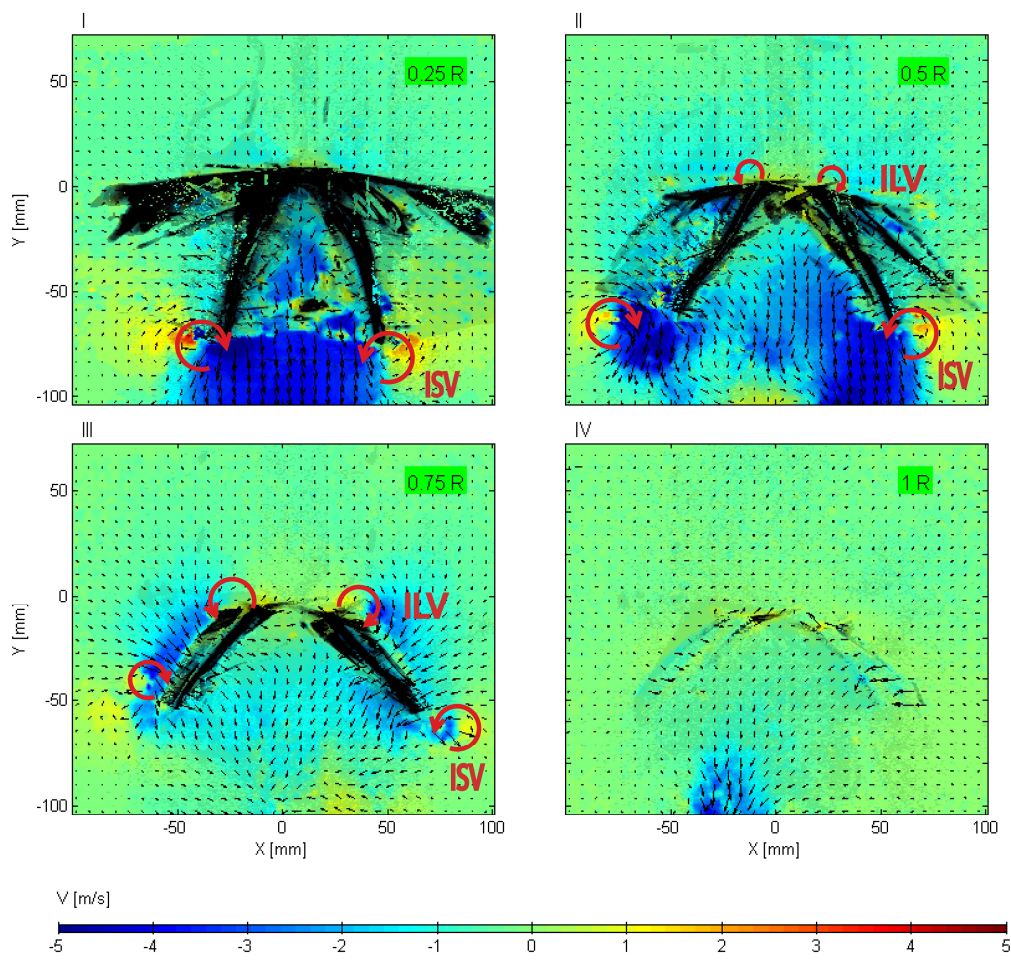

Figure 5.12 Spanwise variation in $\mathrm{V}$-component of velocity during the instroke for $f=13 \mathrm{~Hz}$ and $\tau=0.80$

\subsubsection{Influence of flap frequency}

In this section we investigate the influence of the flapping frequency on the flow field and the forces generated by comparing the time resolved and the phased locked measurements at corresponding dimensionless time instances.

In Figure 5.14 the force fluctuations for both cases are plotted in function of the nondimensional time. At a flapping frequency of $11 \mathrm{~Hz}$, the mean force over one cycle attains $0.15 \mathrm{~N}$, which is not sufficient to lift a DelFly II. The maximal force amounts to $0.54 \mathrm{~N}$ while the second peak value of $0.40 \mathrm{~N}$ is $25 \%$ lower.

Figure 5.13 shows the in-plane velocity component of the 3D vector field of the PIV measurements at a flapping frequency of $11 \mathrm{~Hz}$. The PIV measurements will be compared for the time instances labeled $\mathrm{A}$ to $\mathrm{F}$ in de force plot and the red-colored wing sections in Figure 5.1. As before the color-contours indicate the magnitude of the vertical velocity component. The black regions represent the areas were data are compromised due to shadows, optical obstructions or background reflections.

Compared to frame A of Figure 5.3, the wings deflect less and the wing surfaces peel off earlier. The jet vortices (JV) are generated earlier and are visible further downstream in 
the wake. This could explain why the peak value for in lifting force is reached later at 13 $\mathrm{Hz}$.
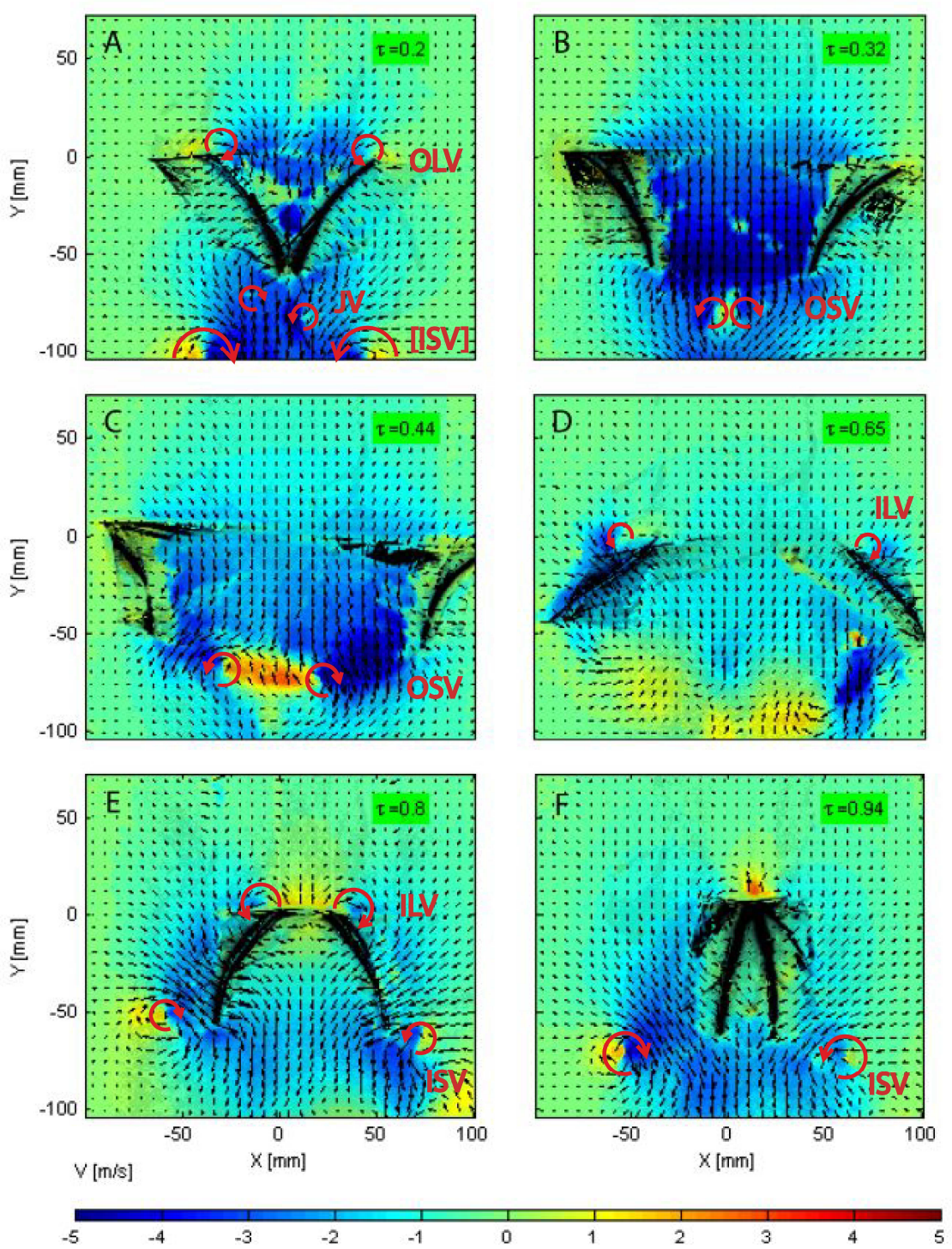

Figure 5.13 Phase locked PIV results for $b=0.75 \mathrm{R}$ and $f=11 \mathrm{~Hz}$

The force plot shows a higher peak value in lifting force at $13 \mathrm{~Hz}$. Conform the analysis of Ellington ${ }^{30}$, the circulation around the exposed chord is proportional to the product of the exposed chord length, the unzipping velocity and a function of the opening angle. This circulation function increases for this range of angles. For the same exposed chord length, the angle between the unzipping wings is lower at a lower flapping frequency. If the unzipping velocity is assumed constant for a constant flapping frequency, a slightly higher circulation would be expected at the higher flapping frequency. Also the heaving motion of the leading edges has higher amplitude at the higher flapping frequency, 


\section{Results}

additionally increasing the circulation, in correspondence to the theory by Lehmann ${ }^{29}$, due to the increases in the relative motion of the wing with respect to the air.

In the translational motion, at frame B, the angle of attack is a little different for both flapping frequencies. Flapping at $11 \mathrm{~Hz}$, the trailing edges have separated and the starting vortices (OSV) are shed earlier in the cycle. Oppositely to what the higher lift values at a flapping frequency of $13 \mathrm{~Hz}$ would suggest, the downwash between the wings flapping at $11 \mathrm{~Hz}$ is stronger. The velocity fields at frame $\mathrm{C}$ of Figure 5.13 reveal that the starting vortices (OSV) left behind in the wake are much stronger and less chaotic at the lower flapping frequency. At this moment the lowest recline in lifting force is reached for the $11 \mathrm{~Hz}$ case. The lift force for the wing flapping at $13 \mathrm{~Hz}$ will decrease even further.

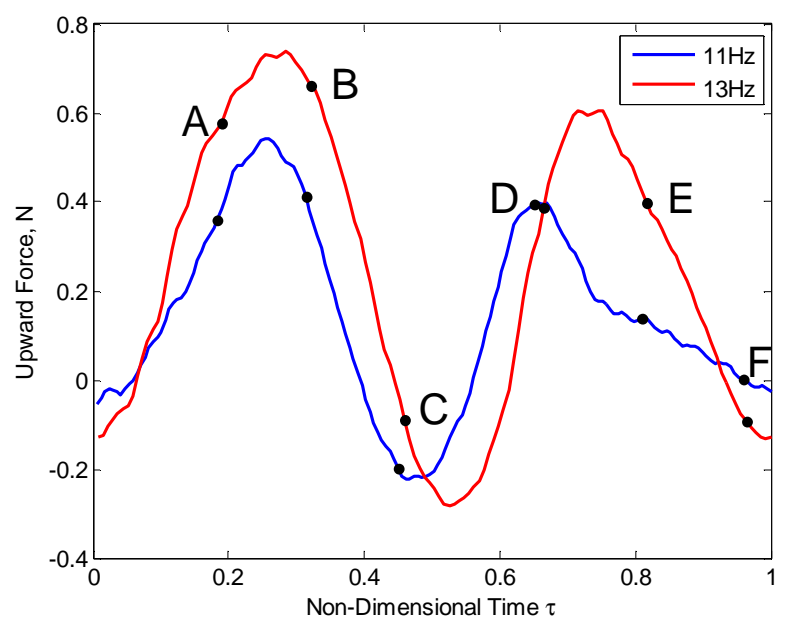

Figure 5.14 Upward force fluctuations during one flap cycle for $f=11 \mathrm{~Hz}$ and $f=13 \mathrm{~Hz}$

Due to the smaller wing surface deflection at the lower flapping frequency, the isolated rotation is faster. The wings have already deflected in frame $\mathrm{D}$, whereas the wings in frame D of Figure 5.3 are still bent. A leading edge vortex (ILV) is generated and the maximal lift force is attained, as is the case at $\tau=0.73$ at the higher flapping frequency (Figure 5.10).

In frame E of Figure 5.13, the angle of attack is larger compared to frame E of Figure 5.3 and the upward expelling jet observed above the leading edges is stronger. A region of high downward velocity is perceived near the outer side of the wing surfaces. This phenomenon might partly explain the large difference in lift force. Little difference can be found in the downward expelled air by the clap between both flapping frequencies. 


\section{Conclusions and recommendations}

PIV velocity measurements and simultaneous force measurements were performed on the DelFly II flapping-wing MAV, to investigate the flow-field behavior and the aerodynamic force generated.

The high flexibility causes the wings to peel and flex during the rotation at the minimal and maximal amplitude respectively. This passive wing deformation largely influences the flow field behaviour.

The wings start peeling apart before the clap has been completed. During the peel, the PIV analysis shows a strong influx between the wings and a conical vortex structure above the leading edges. This peel mechanism contributes significantly to the lift, as revealed by the force measurements.

During the clap, the presence of a mirror wing causes the air to be expelled downwards and to roll up into vortices of the same direction as the bound vorticity of the subsequent stroke. The jet generated by the clap is stronger on the inner half of the wings, but exceptionally weak on the outer half. This is attributed to the high flexibility of the wing foil. The strong and chaotic starting vortex shed at the beginning of the outstroke, is responsible for a strong decline in lift force during the subsequent translational phase. The clap mechanism does rather attenuate than enhance lift.

The occurrence of a leading edge vortex during the flex can not be assessed from the PIV images due to optical obstruction, but is likely to appear since the wing flexing is accompanied with a large increase in lift. An additional augmentation in lift could also be attained by the delayed shedding of a combined starting and stopping vortex. Although this flap phase is accompanied by an increased lift, it does not contribute to the total lift as much as the peel mechanism. The PIV visualization first suggests the occurrence of a leading edge vortex at the onset of the subsequent translational stroke.

Especially during the flex the high deflection of the wings achieved at a higher flapping frequency is favorable. The force plot shows a relatively higher and broader peak in lift force. The aerodynamic mechanism underlying this phenomenon could not be identified.

The results of this study reveal that the most important augmentation in lift generation is due to the peel motion in the current wing configuration of DelFly II. Another significant contribution might be the combined vortex shedding and leading edge vortex strengthening at isolated wing rotation. 
Based on the present findings it is expected that the lift generation could be further enhanced by avoiding the upward expelling air between the leading edges near minimal stroke angle, by extending the momentum jet during the clap along the complete span and by reducing the strength of the starting vortex shed at the onset of the outward translation. An increase in the rotational speed or rigidity of the wings could bring these effects. Future research is recommended to focus on the influence of wing properties and kinematic parameters during the current least effective flapping phase, the clap.

An interesting case could be to provide the wings with cambers, as used for windsurfing sails. These keep the wing under tension and in a predefined shape till the last moment before rotation and flip around fast.

In the design of further experimental campaigns, attention should be paid to the construction of a more robust model to attain a higher repeatability of the measurement series. A better repeatability would bring a solution to the high background reflectivity by preprocessing the image with minimum subtraction. It would also offer the opportunity to reconstruct a three dimensional flow structure from separate measurements. 


\section{Bibliography}

[1] Young, V. (2007) "Science and technology opportunities for unmanned aerial vehicles," European Micro Air Vehicle Conference and Flight Competition, Toulouse

[2] Serokhvostov, S.V. (2008) "Ways and technologies required for MAV miniaturization," European Micro Air Vehicle Conference and Flight Competition, Braunschweig.

[3] De Croon, G.C.H.E., de Clercq, K.M.E., Ruijsink, R., Remes, B. and de Wagter C. (2009) "The DelFly Project," Int. J. MAV (to be published)

[4] Thaweewat, N. (2007) "Influence of wing kinematics on vortex dynamics and performance in insect forward flight. A numerical investigation," Delft University of Technology M.Sc.Thesis

[5] Kupers, G. (2008) "Influence of flexibility on flapping wings at low Reynolds numbers," Delft University of Technology M.Sc.Thesis

[6] Bas, F.M., Lentink, D. and van Oudheusden, B.W. (2008) "Influence of wing kinematics on aerodynamic performance in hovering insect flight" J. Fluid Mech., vol. 594, pp.341-368

[7] Bradshaw, N.L., and Lentink, D. (2008) "Aerodynamic and structural dynamic identification of a flapping wing micro air vehicle", American Institute of Aeronautics and Astronautics, 26th AIAA Applied Aerodynamics Conference

[8] Ellington, C.P. (1995). "Unsteady aerodynamics of insect flight." Biological Fluid Dynamics. ed. C.P. Ellington and T.J. Pedley. Symp. Soc. exp. Biol. 49, 109-129.

[9] Anderson, J.D.(2001) "Fundamentals of aerodynamics", Mc Graw-Hill

[10] Ellington,C.P. (1984) "The aerodynamics of hovering insect flight. IV. Aerodynamic mechanisms" Phil. Trans. R. Soc. Lond. B 305, 79-113

[11] Bomphrey, R.J.; Lawson, N.J.; Harding, N.J.; Taylor, G.K.; Thomas, A.L.R. (2004) "The aerodynamics of manduca sexta: digital particle image velocimetry analysis of the leading-edge vortex" J. Exp. Biol. 208, 1079-1094

[12] Ellington, C.P., Berg, C. van den; Willmott, A.P.; Thomas, A.L.R. (1996) "Leading-edge vortices in insect flight" J. Nature Vol. 384, 19/26, 626-630

[13] Willmot, P.W.; Elington, C.P.; Thomas, A.L.R. (1997) "Flow visualization and unsteady aerodynamics in the flight of the hawkmoth, Manduca Sexta" Phil. Trans.

[14] Van den Berg, C.; Ellington, C.P.(1997) "The three-dimensional leading-edge vortex of a 'hovering' model hawkmoth" Phil. Trans. R. Soc. Lond. B 352, 329-340

[15] Sane, S.P. (2003) "Review: The aerodynamics of insect flight," J. Exp. Biol. 206, 4191-4208

[16] Lehmann, F.O.; Sane, P.S.; Dickinson, M. (2005) "The aerodynamic effects of wing-wing interaction in flapping insect wings" J. Exp. Biol. 208, 3075-3092 
[17] Maxworthy,T. (1979) "Experiments on the Weis-Fogh mechanism of lift gneration by insects in hoveringflight. Part 1. Dynamics of the "fling"' J. Fluid Mech., vol. 93, part 1, pp.47-63

[18] Poelma, C.; Dickson, W.B.; Dickinson, M.H. (2006) "Time-resolved reconstruction of the full velocity field around a dynamically-scaled flapping wing" Experiments in Fluids 41: 213-225

[19] Ellington,C.P. (1984) "The aerodynamics of hovering insect flight. III. Kinematics" Phil. Trans. R. Soc. Lond. B 305, 79-113

[20] Srygley, R. B. and Thomas, A. L. R. (2002) "Unconventional lift-generating mechanisms in free-flying butterflies," Nature 420, 660-664

[21] Lehmann, F.O., Pick, S., "The aerodynamic benefit of wing-wing interaction depends on stroke trajectory in flapping insect wings", Journal of Experimental Biology, Volume 210, pp. 1362-1377, 2007.

[22] "Design of a flapping wing vision-based Micro-UAV," Design synthesis exercise 2005 TUDelft

[23] Raffel, M.; Willert, C.; Wereley, S. and Kompenhans, J. (2007) "Particle Image Velocimetry: a practical guide," Springer, second edition.

[24] http://www.dantecdynamics.com/

[25] Lawson, N.J. and Wu, J. (1997) "Three-dimensional particle image velocimetry: error analysis of stereoscopic techniques," Meas. Sci. Technol. 8 894-900

[26] Westerweel, J., "Efficient detection of spurious vectors in particle image velocimetry data," J. Exp. Fluids, Vol. 16, 1994, pp. 236-247.

[27] Dubief, Y. and Delcayre, F. (2000) "On coherent-vortex identification in turbulence," J. Turbulence 1:1-22

[28] Jeong, J. and Hussain, F. (1995) "On the identification of a vortex," J. Fluid Mech. Vol. 285, pp. 69-94

[29] Lehmann, F.O., Pick, S., "The aerodynamic benefit of wing-wing interaction depends on stroke trajectory in flapping insect wings", Journal of Experimental Biology, Volume 210, pp. 1362-1377, 2007.

[30] Ellington,C.P. (1984) "The aerodynamics of hovering insect flight. IV. Aerodynamic mechanisms" Phil. Trans. R. Soc. Lond. B 305, 79-113

[31] Birch, J.M; Dickson, B.D.; Dickinson, M.H. (2004) "Force production and flow structure of the leading edge vortex on flapping wings at high and low Reynolds numbers" J. Exp. Biol. 207, 1063-1072

[32] Aono, H.,Liu, H."A numerical study of hovering aerodynamics in flapping insect flight"

[33] Holten, T. van and Melkert, J.A. (1994) "Prestaties en vliegeigenschappen van hefschroefvliegtuigen," TUDelft lecture notes 


\section{Appendix}

\section{A. Reynolds number of flapping flight}

For the Reynolds number, which is the ratio of inertial to viscous forces, is defined as the product of a characteristic length and velocity divided by the kinematic viscosity $v$ of the fluid. The influence of the Reynolds number on the LEV has been demonstrated by experiments by Birch $^{31}$ and numerical by Aono ${ }^{32}$.

Several characteristic scales are used in literature on flapping flight. Most commonly, the Re for hovering flight is defined based on the mean wing chord $\bar{c}$ and the mean wing tip velocity $V_{t}$.

$\operatorname{Re}=\frac{\overline{V_{t}} \cdot \bar{c}}{v}$

The definition of these parameters is adapted from (Ellington et al., 1999 \& 2001) in which $\mathrm{R}$ is the wing length, $\mathrm{S}$ the planform area of both wings, $\mathrm{AR}$ the aspect ratio, $\mathrm{f}$ the wing beat frequency and $\Phi$ the wing beat amplitude.

$\bar{c}=\frac{2 R}{A R}=\frac{S}{2 R}$ since $A R=\frac{4 R^{2}}{S}$

$V_{t}=2 \phi f R$

For large variations in aspect ratio, the Re based on the induced velocity is more suitable. Regarding the insect's stroke plane as an actuator disc, this velocity component can be derived from the actuator disc theory33. The force generated by the actuator disc equals the product of induced velocity at the disc location and the mass flux across the disc area. Since the acceleration is generated partly in front of the disc, the velocity on the disc is half of the total induced velocity. During hover this would be given by

$W=2 m V_{\text {ind }}$,

where the force generated equals the total body weight and $m$ is the mass flux of air being accelerated to a velocity $\mathrm{V}_{\text {ind. }}$. This leads to the expression of the induced velocity $V_{\text {ind }}=\sqrt{W / 2 \rho A}$,

where $\rho$ is the air density and A is the disc area. This expression contains the assumption of uniform downward acceleration of the fluid across the entire area. Real flapping wings have a non-uniformity for example caused by losses due to tip and root vortices, variations in wing twist and planform, turbulences near the body etc. Since any knowledge of the specific downwash distribution of any insect or DelFly is missing, this assumption is necessarily applied. For comparative reasons this assumption is not limiting. 


\begin{tabular}{|c|c|c|c|c|c|c|}
\hline & & & DelFly & Hawkmoth & Bat & FruitFly \\
\hline Reference & & & & $\begin{array}{l}\text { Ellington } \\
2001\end{array}$ & $\begin{array}{l}\text { Muijres } \\
2008\end{array}$ & $\begin{array}{l}\text { Poelma } \\
2006\end{array}$ \\
\hline \multicolumn{7}{|l|}{ Wing planform } \\
\hline Wing span & {$[\mathrm{m}]$} & $b$ & 0,140 & 0,051 & 0,242 & 0,003 \\
\hline Maximal chordlength & {$[\mathrm{m}]$} & $c_{\max }$ & 0,081 & & & 0,001 \\
\hline Number of wings & {$[-]$} & $n$ & 4 & 2 & 2 & 2 \\
\hline Maximal flap angle & {$\left[{ }^{\circ}\right]$} & $\phi$ & 48 & 120 & 34 & 180 \\
\hline Wingbeat frequency & $\begin{array}{l}{[\mathrm{Hz}} \\
]\end{array}$ & $f$ & 13 & 25 & 10 & 200 \\
\hline Weight & [ $[\mathrm{kg}$ & $W$ & 0,0178586 & 0,002 & 0,0105 & \\
\hline Wing surface & {$\left[\mathrm{m}^{2}\right.$} & $S$ & 0,010 & & 0,009 & \\
\hline Aspect ratio & {$[-]$} & $A R=\frac{b^{2}}{S}$ & 1,93 & 1,42 & 6,35 & \\
\hline Mean chordlength & {$[\mathrm{m}]$} & $\bar{c}=\frac{S}{b}=\frac{b}{A R}$ & 0,073 & 0,036 & 0,039 & \\
\hline tip-to-tip amplitude & {$[\mathrm{m}]$} & $A=b \sin \phi$ & 0,104 & 0,044 & 0,136 & 0,006 \\
\hline "rotor surface" & $\begin{array}{l}{\left[\mathrm{m}^{2}\right.} \\
]\end{array}$ & $M=b^{2} \phi \frac{n}{2}$ & 0,03 & 0,01 & 0,03 & $2,83 \mathrm{E}-05$ \\
\hline $\begin{array}{l}\text { Flap velocity at } \\
\text { wingtip }\end{array}$ & $\begin{array}{l}{[\mathrm{m} /} \\
\mathrm{s}]\end{array}$ & $U_{t i p}=2 \phi f b$ & 3,05 & 5,34 & 2,89 & 3,80 \\
\hline Induced velocity & $\begin{array}{l}{[\mathrm{m} /} \\
\mathrm{s}]\end{array}$ & $U_{\text {ind }}=\sqrt{\frac{W g}{2 \rho M}}$ & 1,47 & 1,21 & 1,09 & \\
\hline Effective velocity & $\begin{array}{l}{[\mathrm{m} /} \\
\mathrm{s}]\end{array}$ & $\begin{array}{l}\overrightarrow{U_{\text {eff }}}=\overrightarrow{U_{\text {ind }}} \\
+\overrightarrow{U_{\text {flap }}}+\overrightarrow{U_{\infty}}\end{array}$ & 3,20 & 4,63 & 2,82 & \\
\hline \multirow[t]{4}{*}{ Reynolds number } & {$[-]$} & $\operatorname{Re}=\frac{\bar{c} U_{t i p}}{v}$ & 15289 & 13275 & 7669 & \\
\hline & {$[-]$} & $\operatorname{Re}=\frac{c_{\max } U_{t i p}}{v}$ & 17035 & & & 257 \\
\hline & {$[-]$} & $\operatorname{Re}=\frac{\bar{c} U_{\text {ind }}}{v}$ & 7383 & 3008 & 2906 & \\
\hline & {$[-]$} & $\operatorname{Re}=\frac{\bar{c} \overline{U_{f l a p}}}{v}$ & 7644 & & 5000 & \\
\hline
\end{tabular}

From reference

Derived from given data 


\section{B. V-component of time resolved PIV measurements for different time instants and spanwise locations}
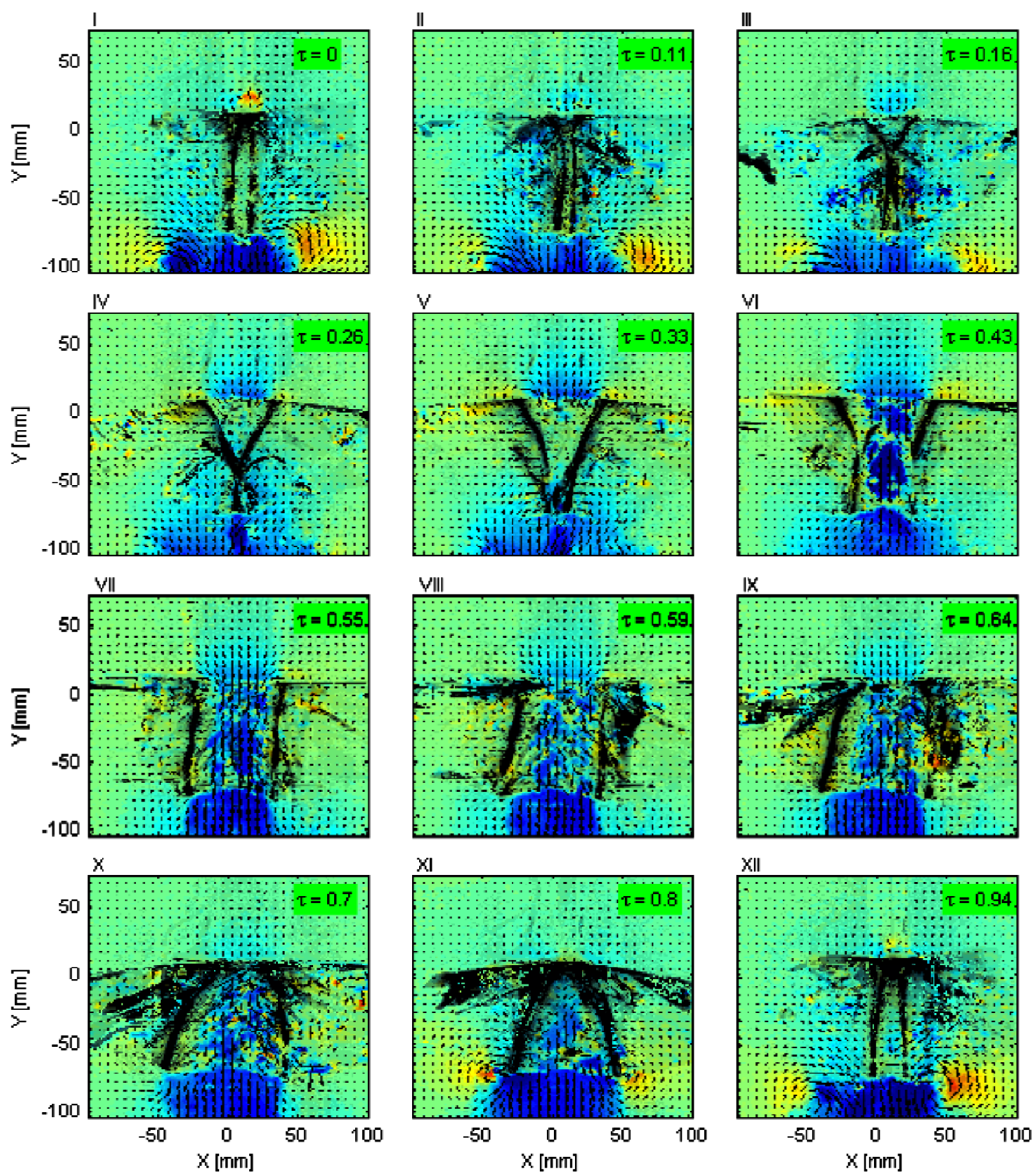

$\mathrm{V}$ [m/s]

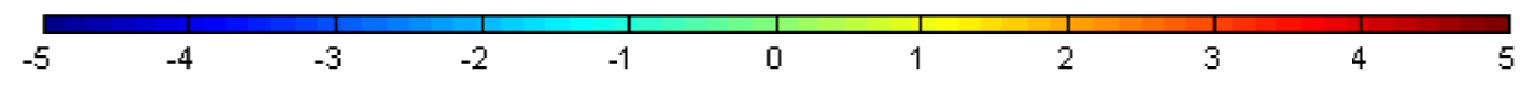

Figure B.1 V-component of velocity for $0.25 \mathrm{R}$ and $f=13 \mathrm{~Hz}$ 

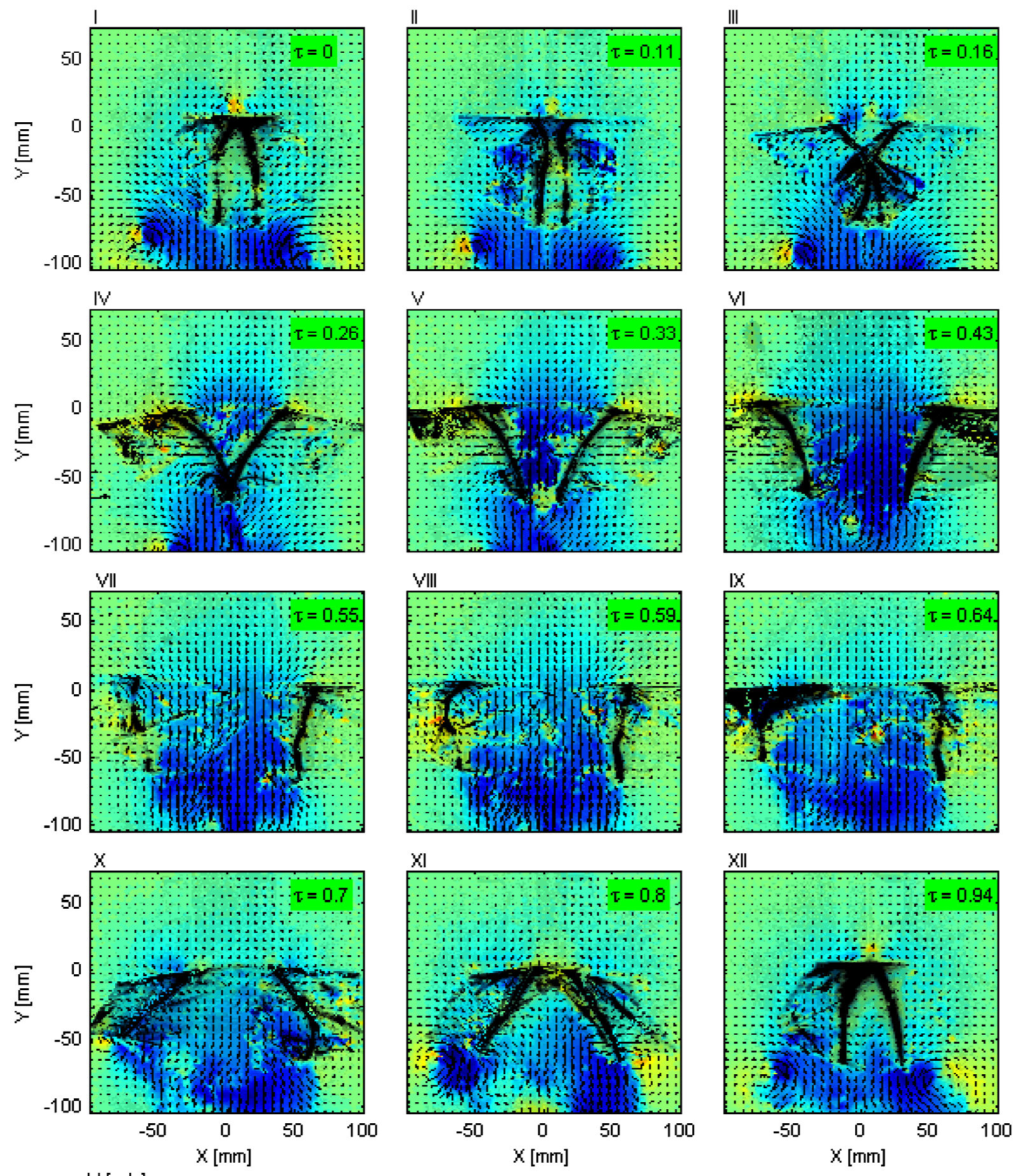

\section{$\mathrm{V}$ [m/s]}

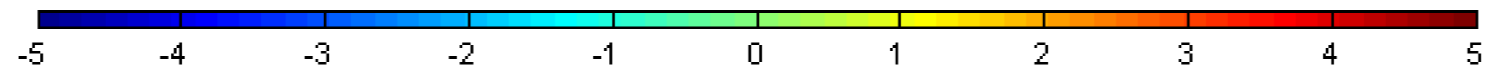

Figure B.2 V-component of velocity for $0.50 \mathrm{R}$ and $f=13 \mathrm{~Hz}$ 

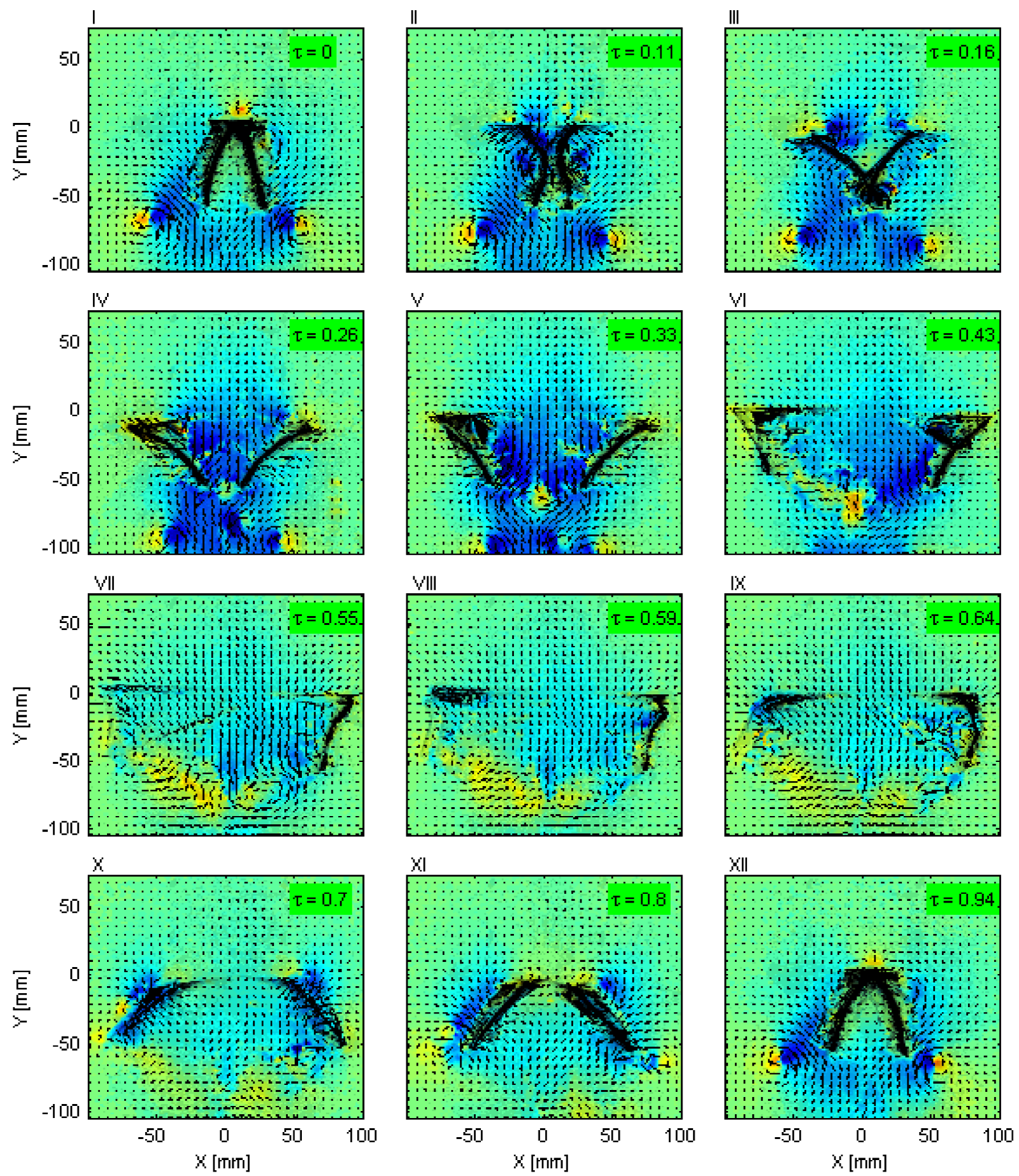

$\mathrm{V}[\mathrm{m} / \mathrm{s}]$

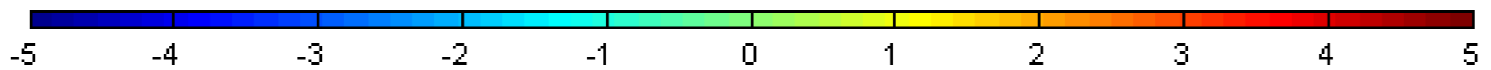

Figure B.3 V-component of velocity for $0.75 R$ and $f=13 \mathrm{~Hz}$ 

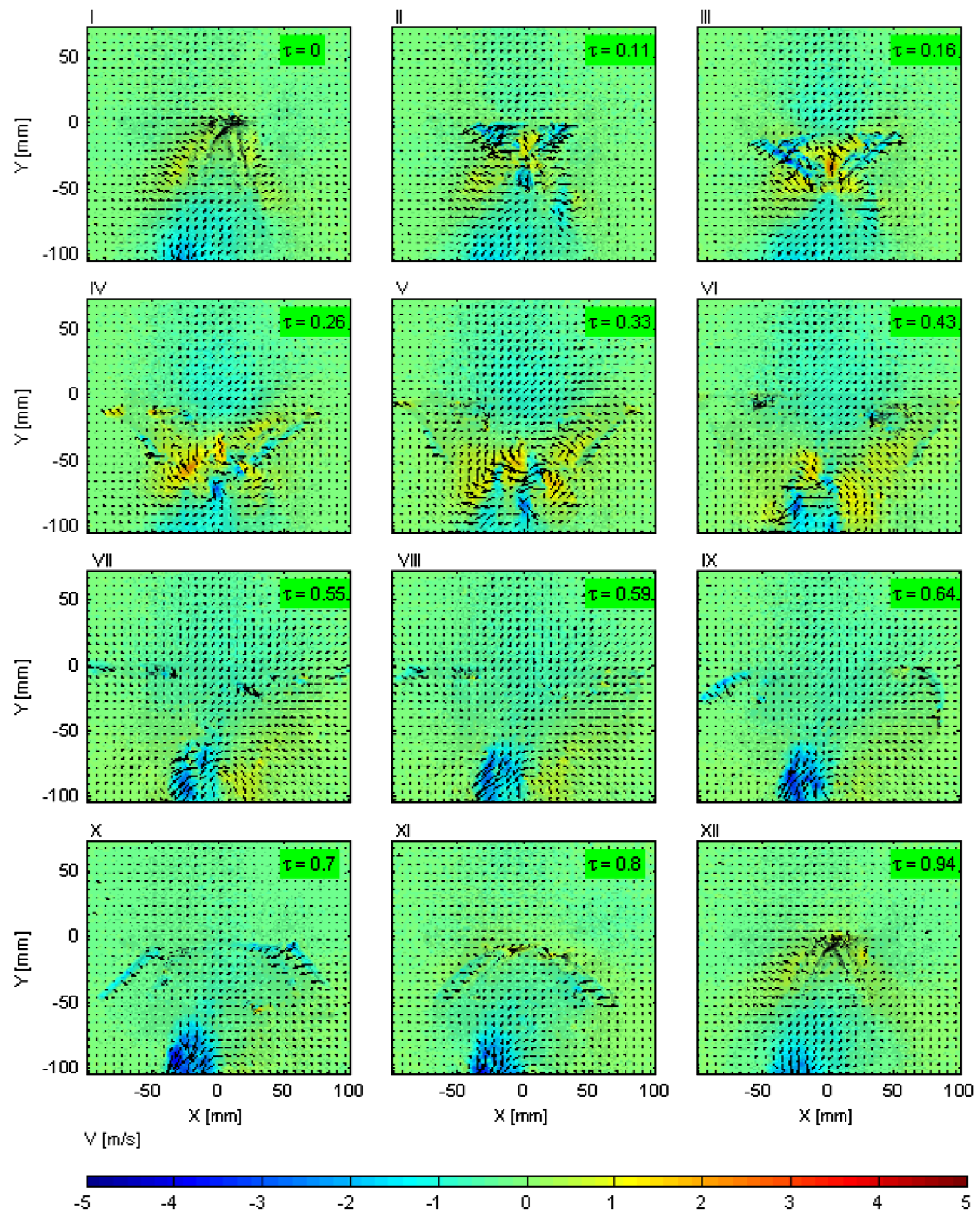

Figure B.4 V-component of velocity for $1.00 R$ and $f=13 \mathrm{~Hz}$ 


\section{Vortex visualization of time resolved PIV measurements for different time instants and spanwise locations}
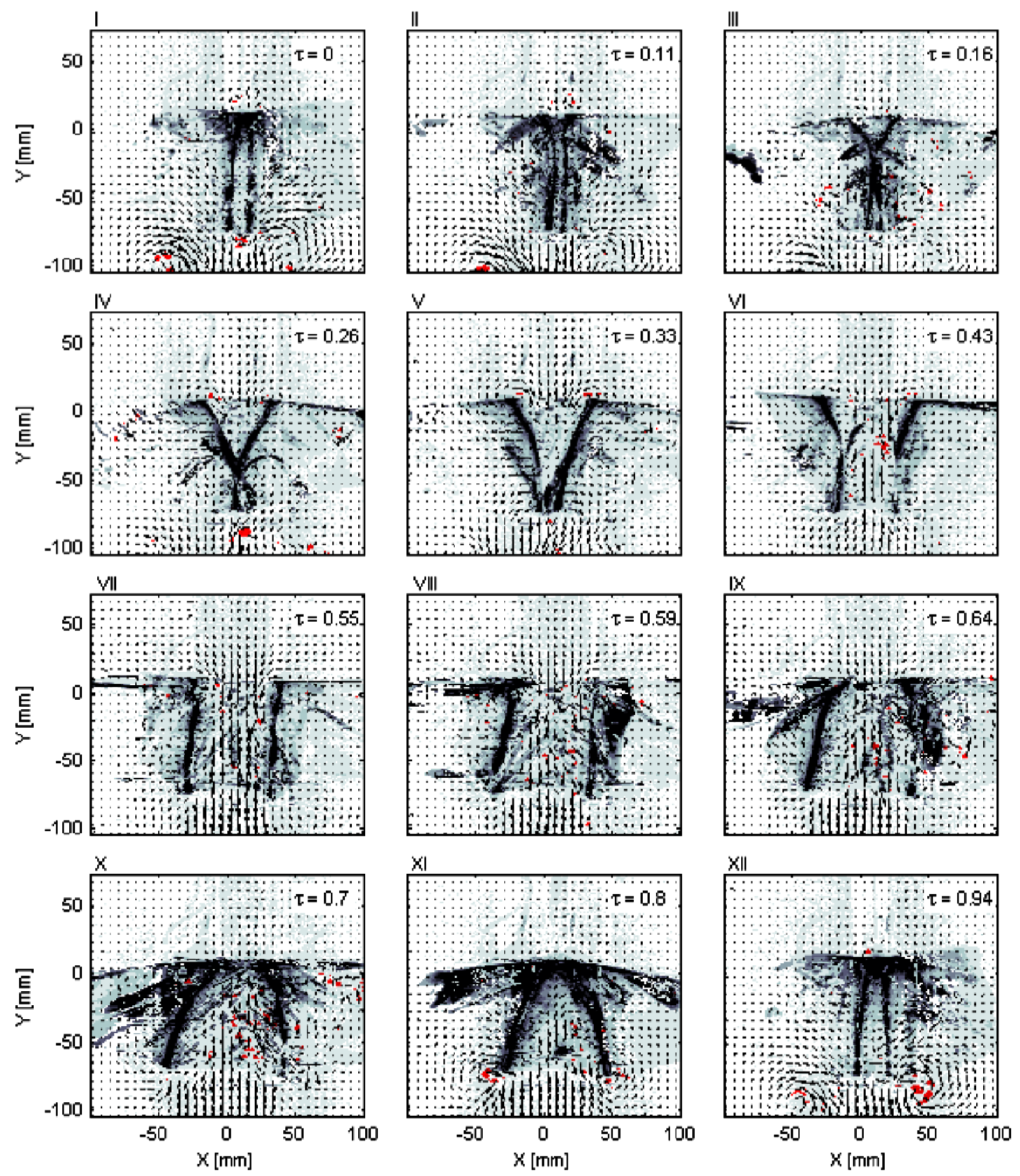

Figure C.1 vortex identification for $0.25 R$ and $f=13 \mathrm{~Hz}$ 

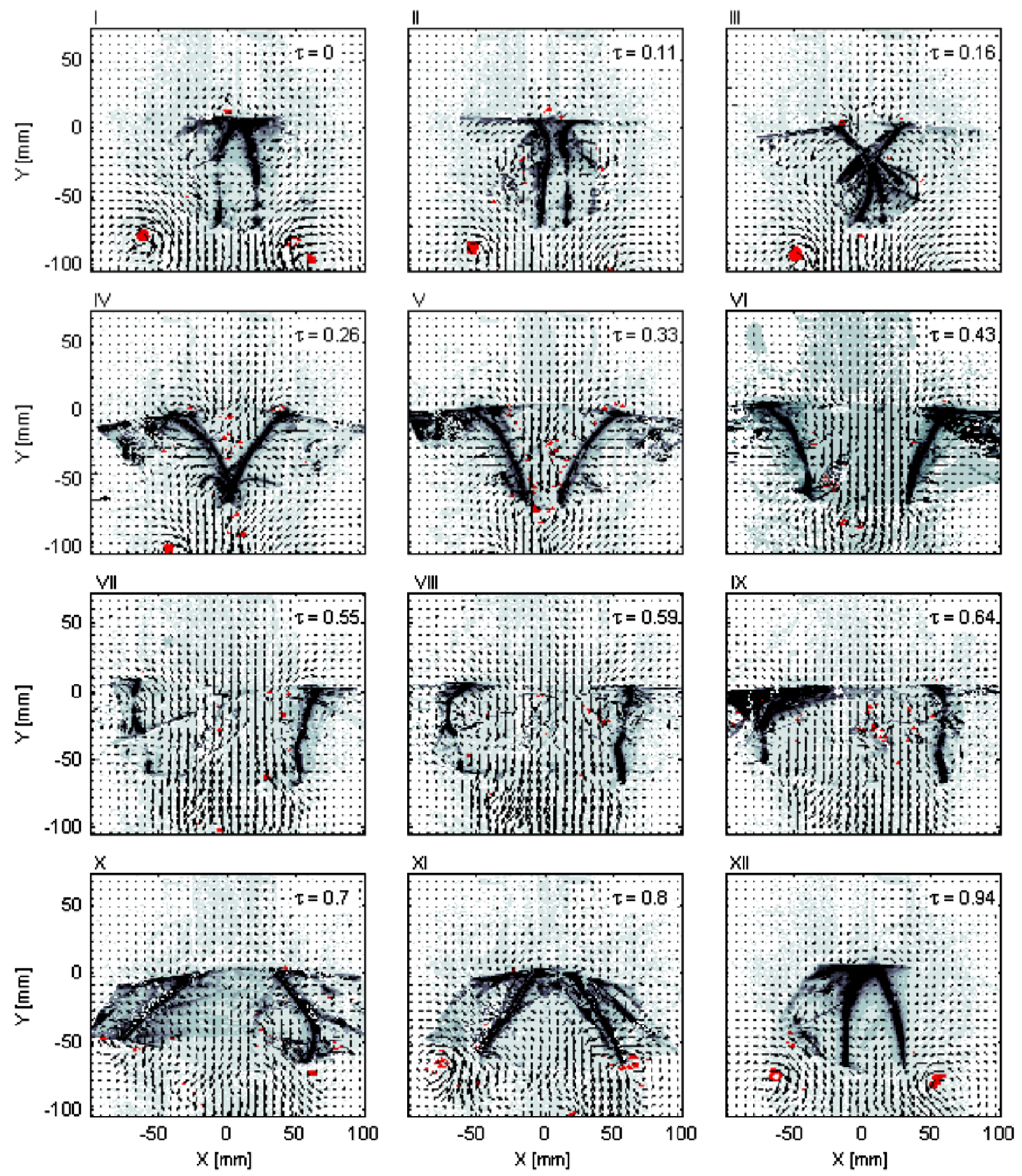

Figure C.2 vortex identification for $0.50 R$ and $f=13 \mathrm{~Hz}$ 

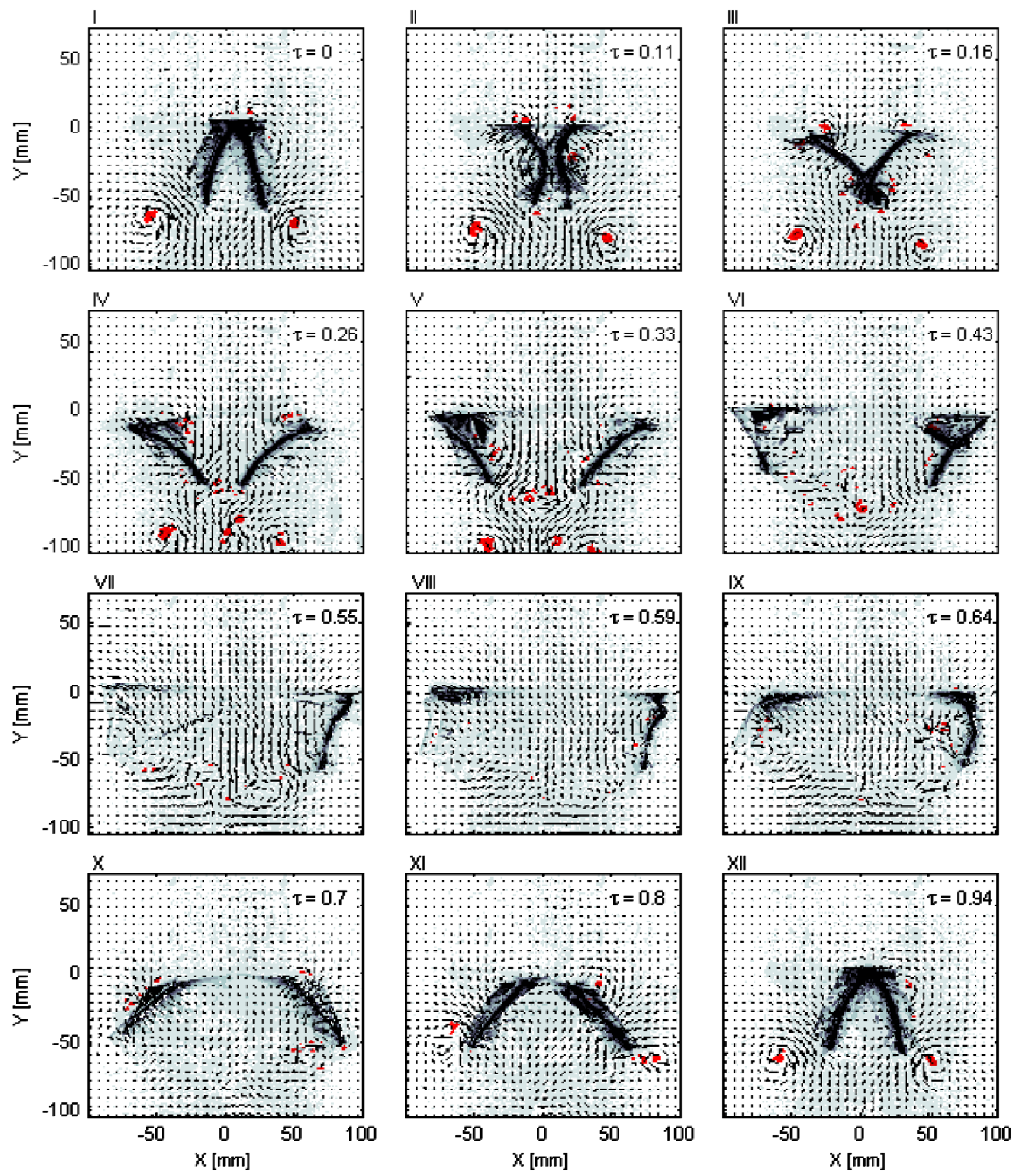

Figure C.3 vortex identification for $0.75 R$ and $f=13 \mathrm{~Hz}$ 

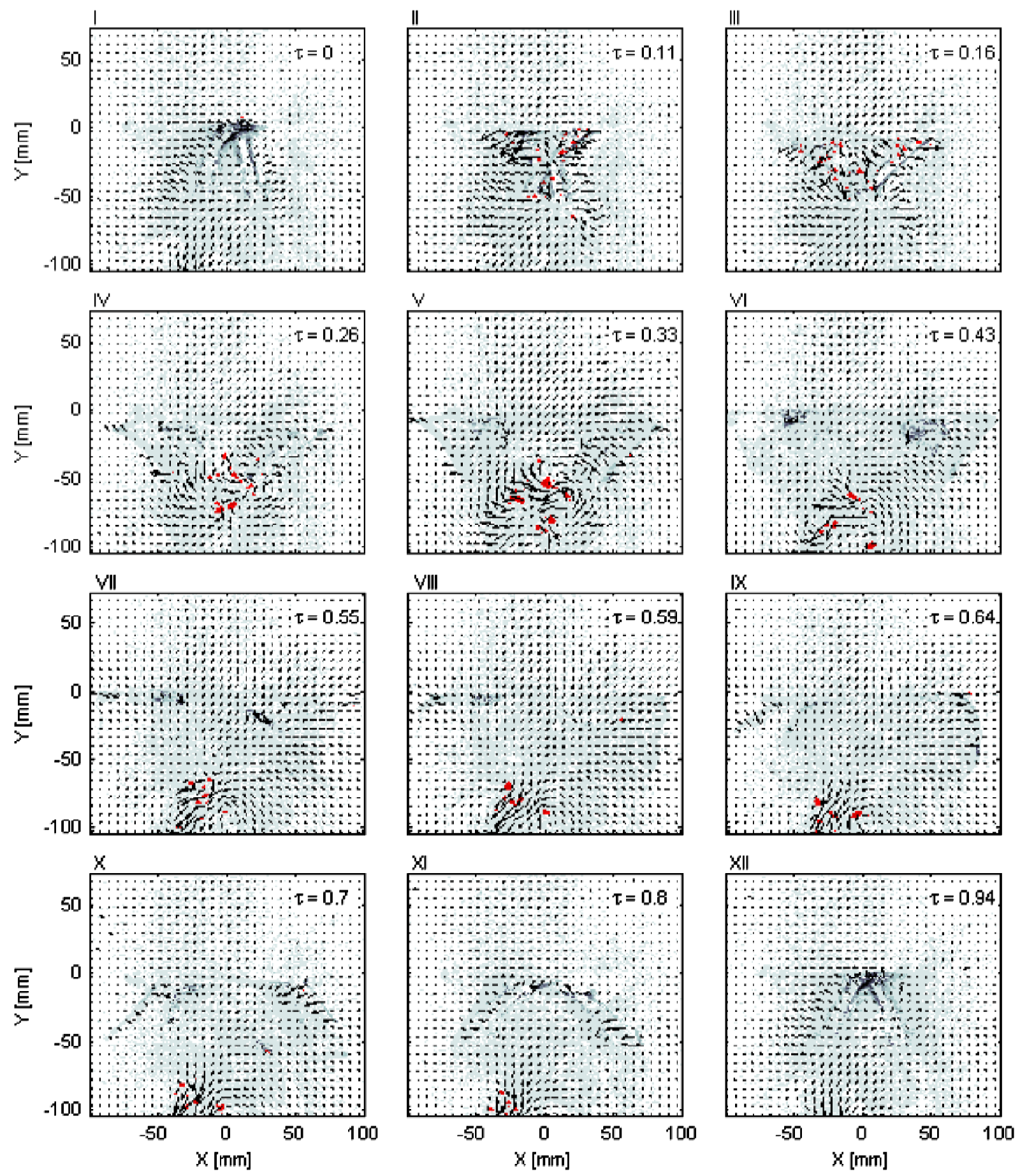

Figure C.4 vortex identification for $1.00 R$ and $f=13 \mathrm{~Hz}$ 


\section{D.Vortex visualization of phase locked PIV measurements}
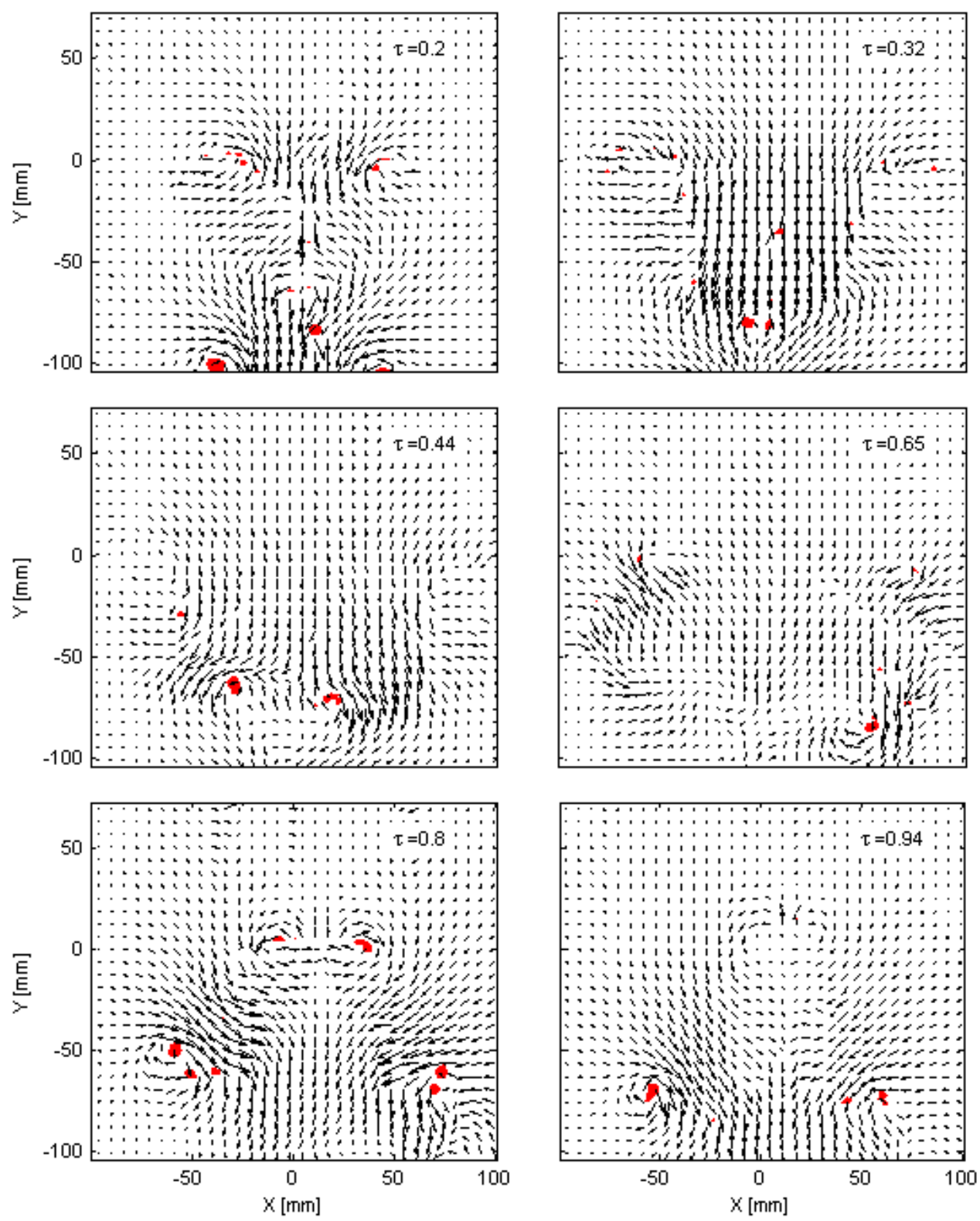

Figure D.1 Vortex Identification for $0.75 \mathrm{R}$ and $\mathrm{f}=11 \mathrm{~Hz}$ 


\section{E. V-component of time resolved PIV measurements zoomed in on clap and fling for different time instants and spanwise locations}
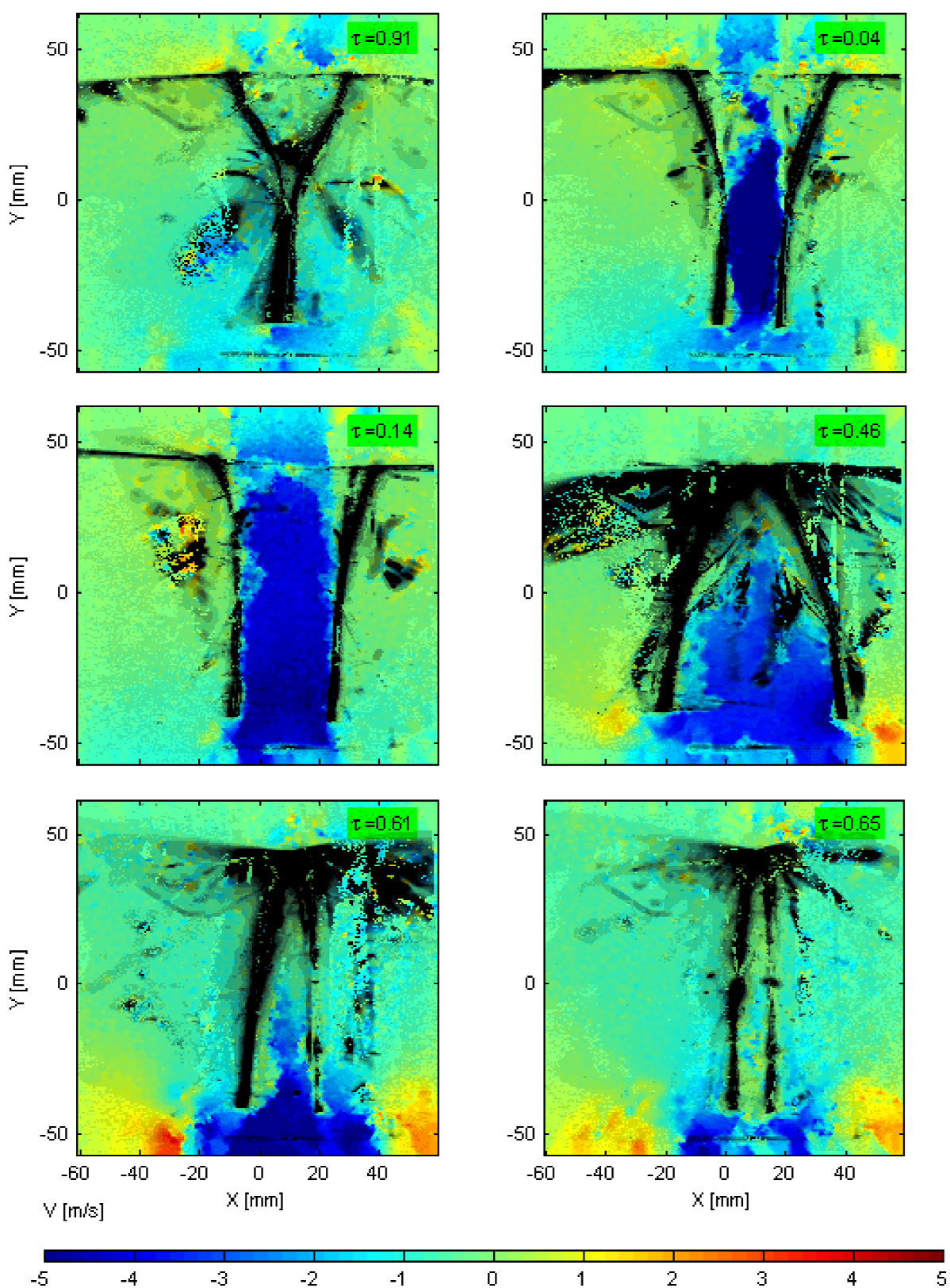

Figure E.1 V-component of the velocity for $0.25 R$ and $f=13 \mathrm{~Hz}$ 

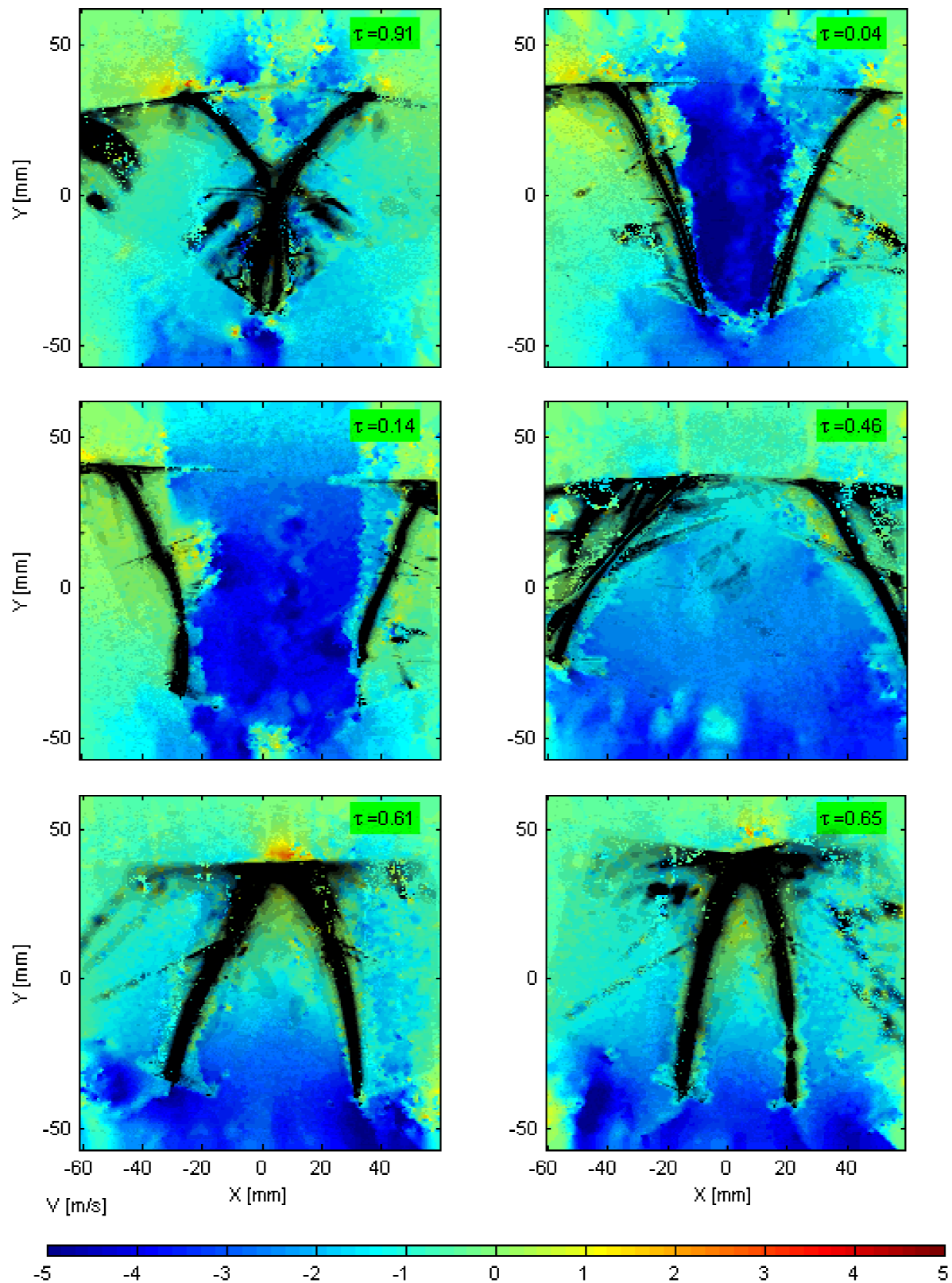

Figure E.2 V-component of the velocity for $0.50 \mathrm{R}$ and $f=13 \mathrm{~Hz}$ 

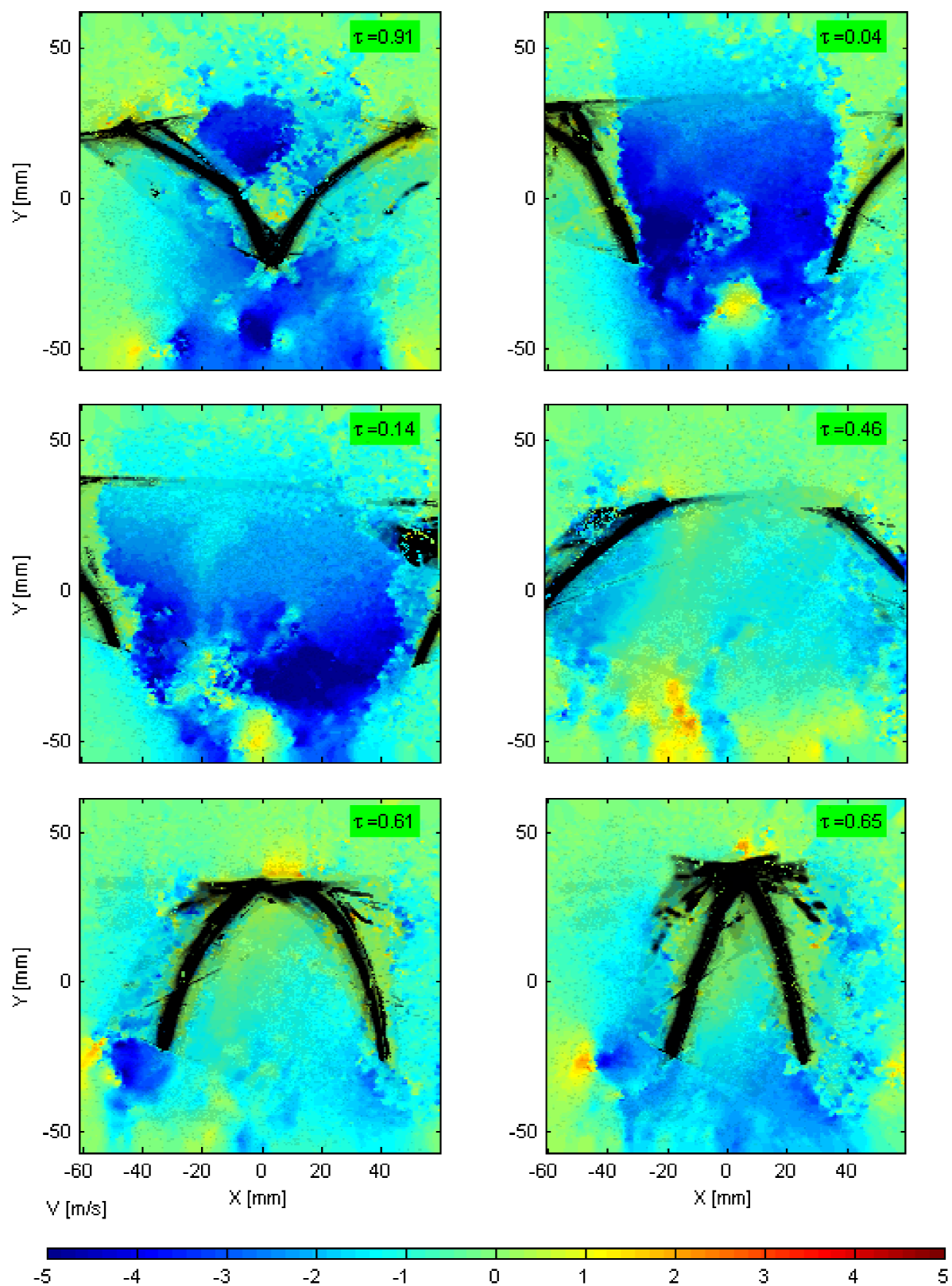

Figure E.3 V-component of the velocity for $0.75 \mathrm{R}$ and $\mathrm{f}=13 \mathrm{~Hz}$ 

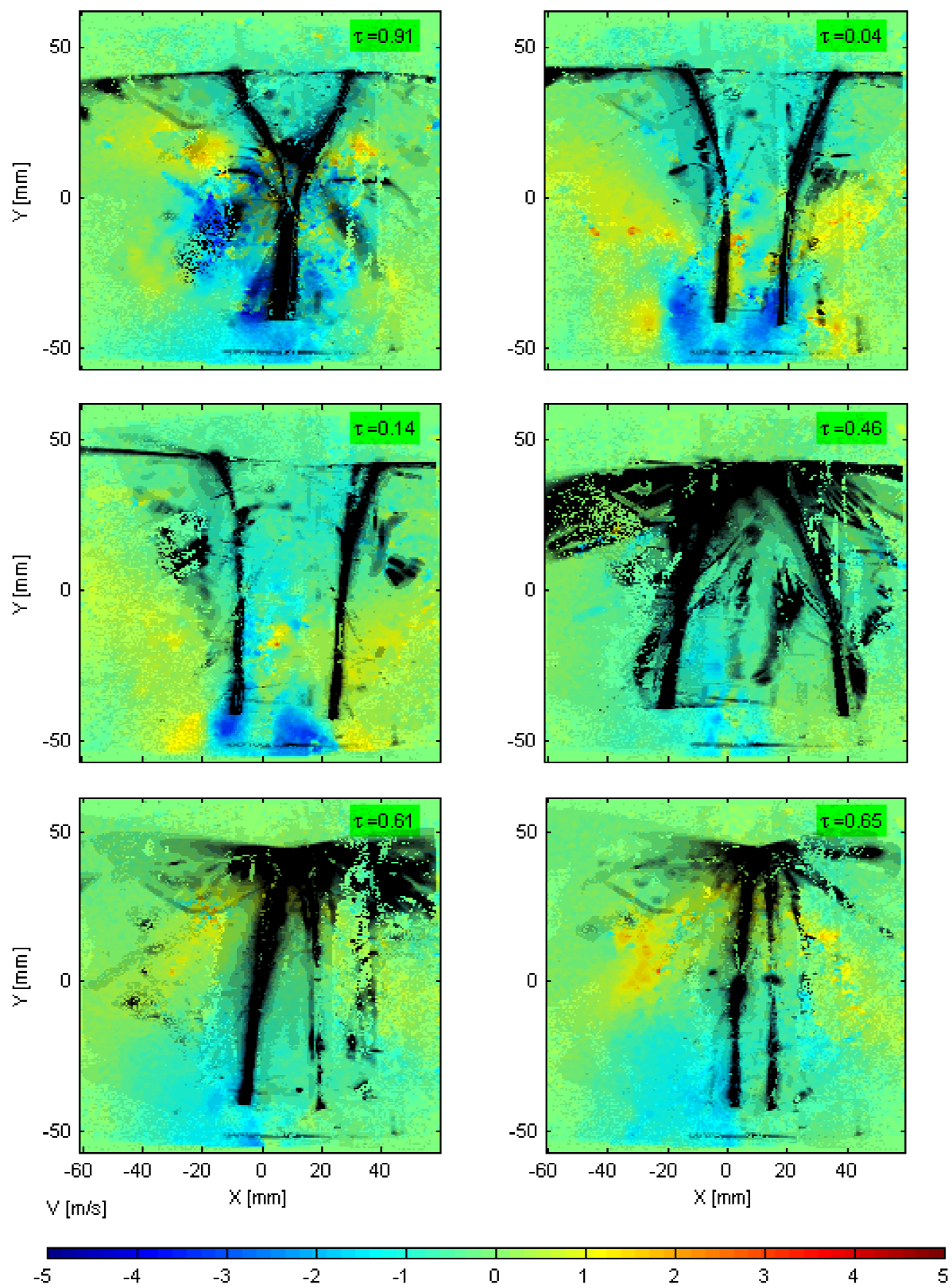

Figure E.4 V-component of the velocity for $1.00 R$ and $f=13 \mathrm{~Hz}$ 


\section{F. Vortex visualization of time resolved PIV measurements in the wake for different spanwise locations}
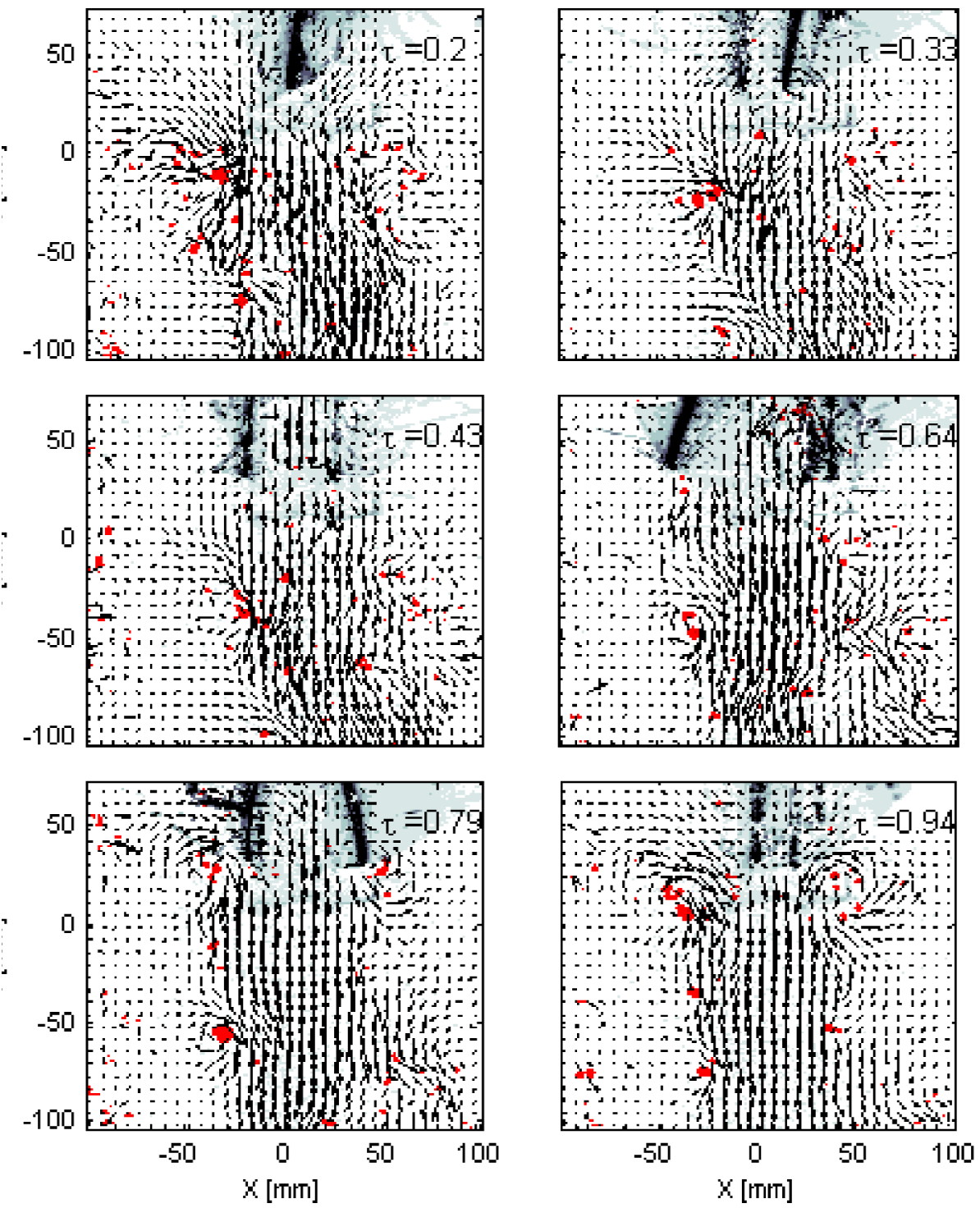

Figure F.1 vortex identification in the wake for $0.25 R$ and $f=13 \mathrm{~Hz}$ 

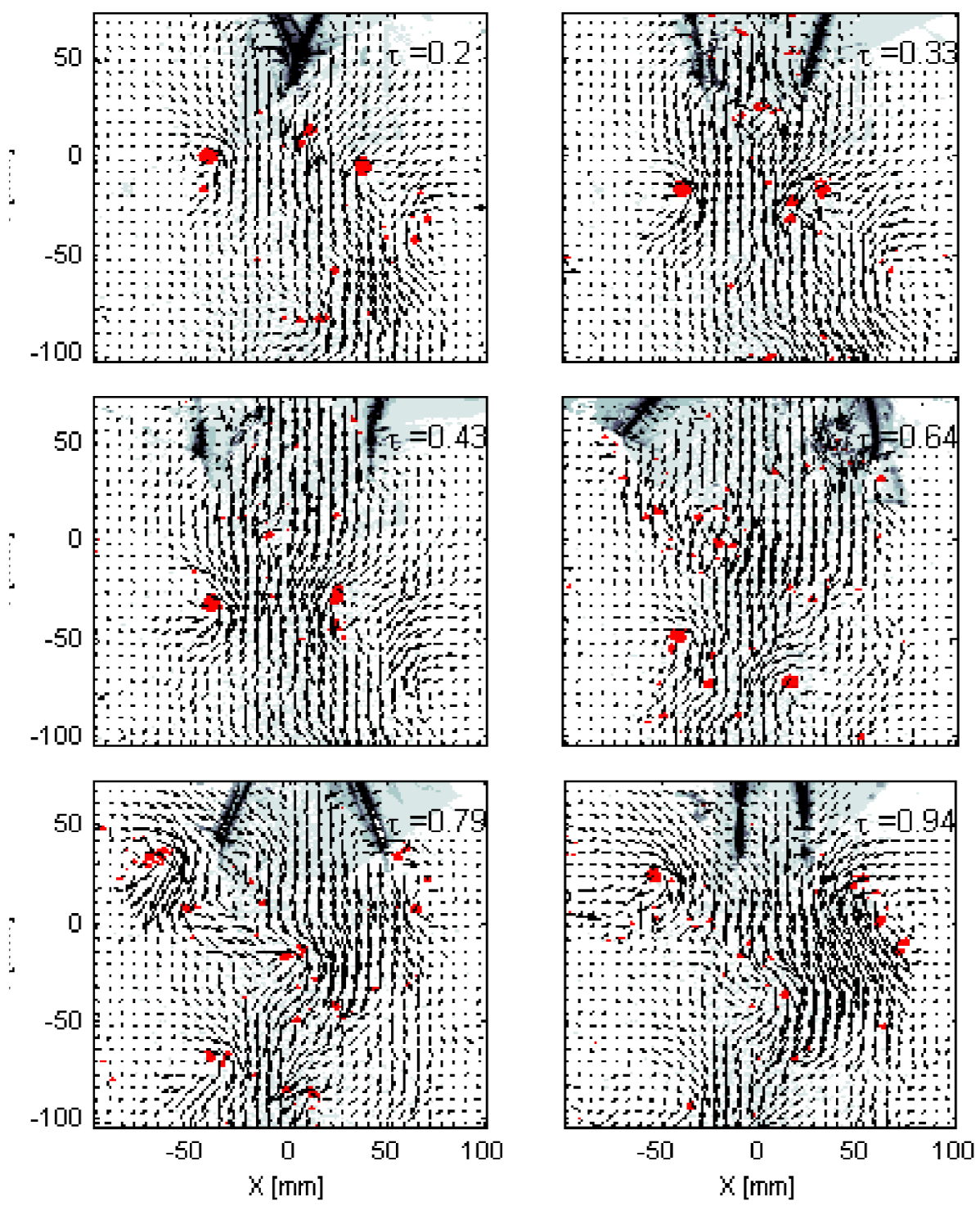

Figure $F .2$ vortex identification in the wake for $0.50 \mathrm{R}$ and $\mathrm{f}=13 \mathrm{~Hz}$ 

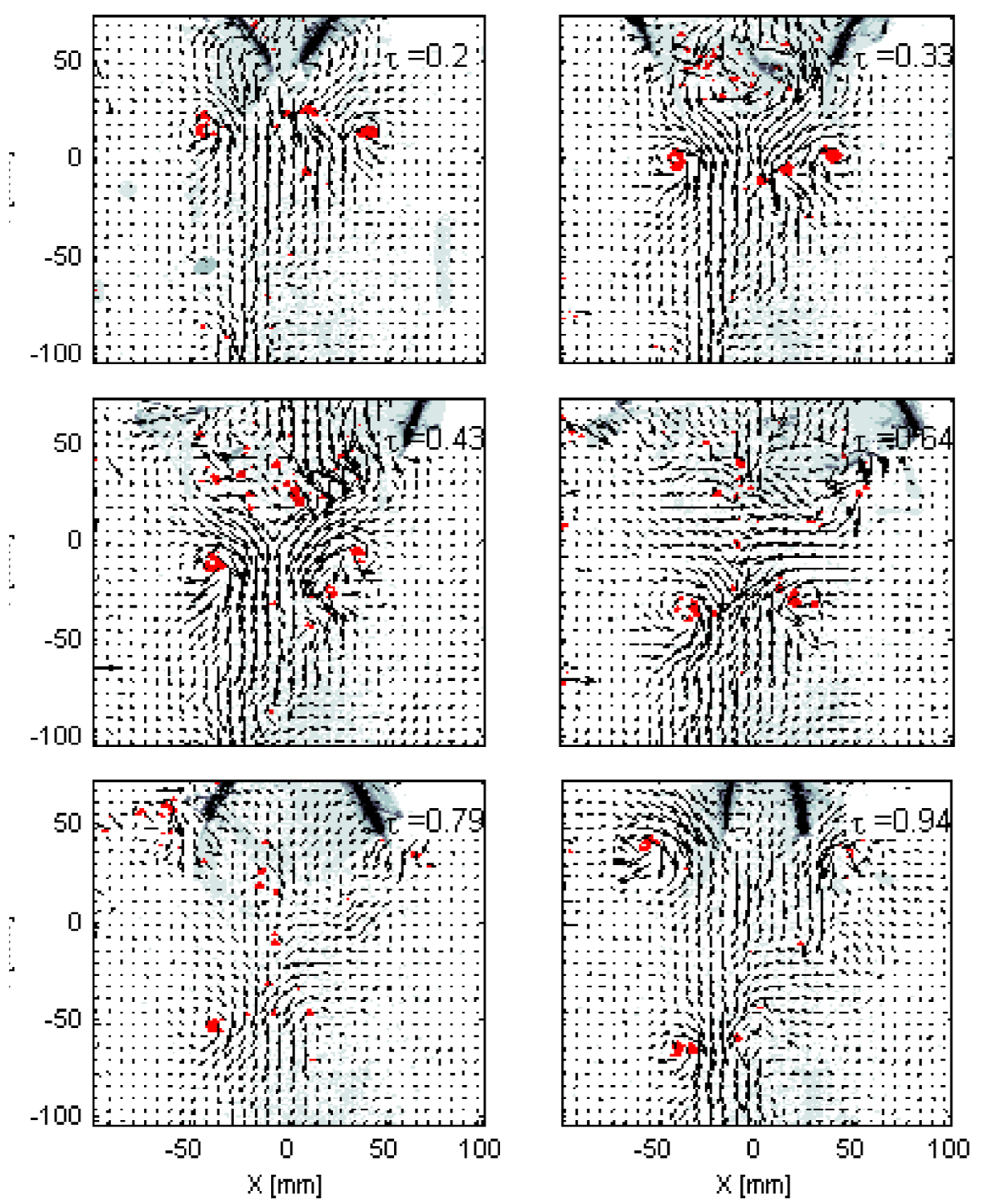

Figure F.3 vortex identification in the wake for $0.75 R$ and $f=13 \mathrm{~Hz}$ 

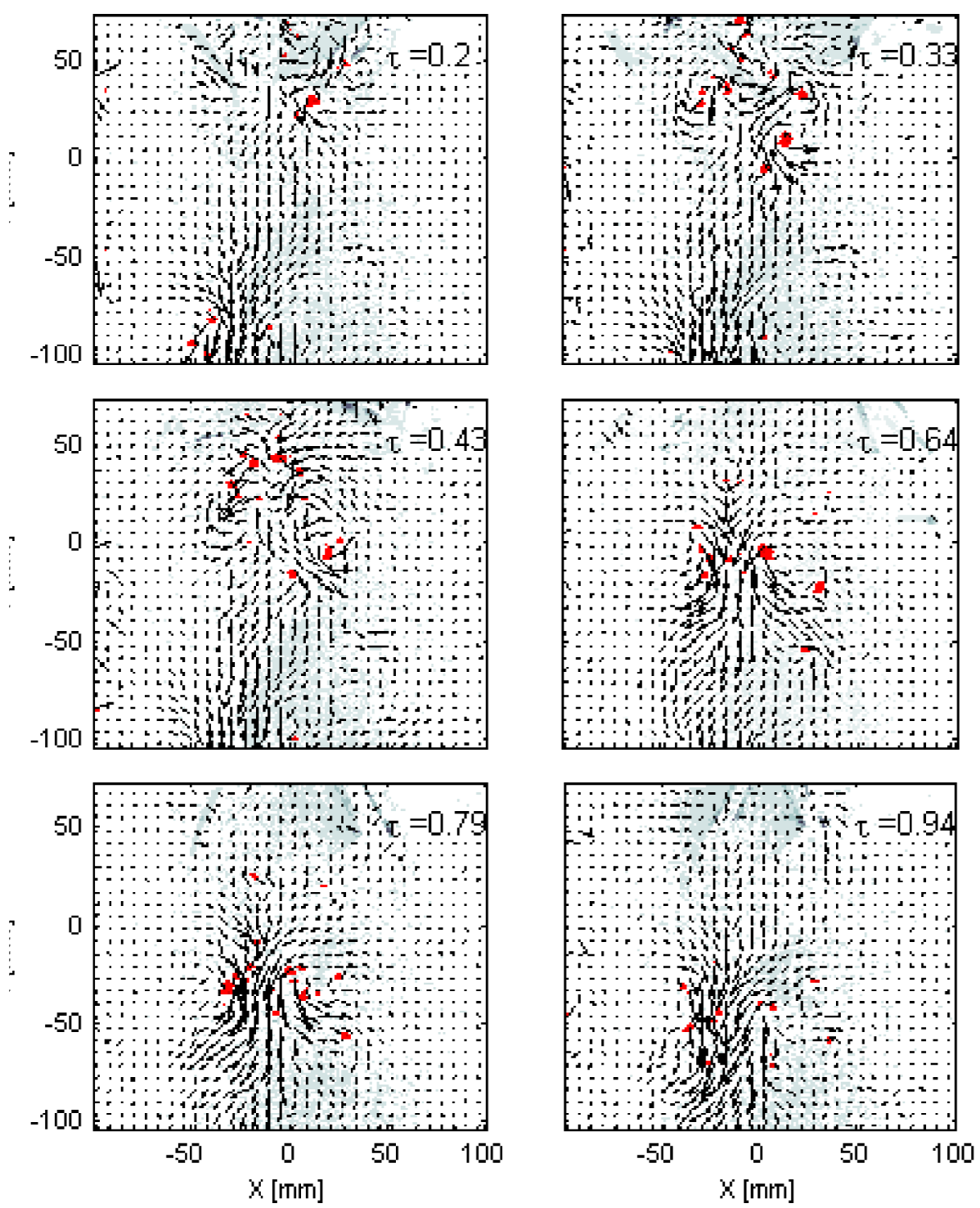

Figure F.4 vortex identification in the wake for $1.00 R$ and $f=13 \mathrm{~Hz}$ 



